\title{
Aglomerados de pentaceno e nanotubos de carbono: um estudo MM/MQ (Mecânica Molecular/Mecânica Quântica)
}

\author{
Antonio Claudio Michejevs Padilha
}

Orientador: Prof ${ }^{a}$. Dr ${ }^{\mathrm{a}}$. Maria Cristina dos Santos

Dissertação apresentada ao Instituto de Física da Universidade de São Paulo para a obtenção do título de Mestre em Ciências.

Comissão examinadora:

Prof $^{\mathrm{a}}$. Dr ${ }^{\mathrm{a}}$. Maria Cristina dos Santos (IFUSP)

Prof $^{\mathrm{a}}$. Dr ${ }^{\mathrm{a}}$. Ana Claudia Monteiro Carvalho (UNIFEI)

Prof $^{\mathrm{a}}$. Dra ${ }^{\mathrm{a}}$. Rosangela Itri (IFUSP) 
Padilha, Antonio Claudio Michejevs

Aglomerados de pentaceno e nanotubos de carbono: um estudo $\mathrm{MM} / \mathrm{MQ}$ (mecânica molecular/mecânica quântica) - São Paulo, 2011.

Dissertação (Mestrado) - Universidade de São Paulo. Instituto de Física - Depto. de Física de Materiais e Mecânica

Orientador: Profa. Dra. Maria Cristina dos Santos

Área de Concentração: Física da Matéria Condensada

Unitermos: 1.Física da Matéria Condensada; 2. Física Atômica; 3.Física Molecular; 4. Estrutura da Matéria (Física Moderna); 5. Mecânica Clássica 
À Sayuri, por tudo. 

O objetivo é o caminho.

Provérbio Taoísta 



\section{Agradecimentos}

Antes de qualquer um, agradeço à minha orientadora, $\operatorname{Prof}^{a} \operatorname{Dr}^{a}$ Maria Cristina do Santos, que acompanhou todo este trabalho, dando conselhos, sugestões e até broncas, mas nunca esquecendo seu papel: o de orientar. Serei sempre grato por todo o tempo e energia dedicados à minha orientação.

Amigos e colegas como Jeconias Guimarães, Elton Carvalho, Rodrigo Castellanos, Fabricio Marques, Felipe Villaverde, Rafael Rodrigues, Filipe Dalmatti, Philippe Petersen, Jorgivan Dias, Ricardo Sugawara e Rafael Morgado contribuiram para este trabalho de diversas formas. Agradeço enormemente o apoio de todos.

A meus pais, Tatiana e Antonio, por me ofereceram condições para que pudesse ir atrás do que acredito, meu irmão Yuri por ser um grande amigo e companheiro e minha avó Izaura por sempre se dedicar enormemente à minha formação, meu sincero muito obrigado.

Por todo o amor e compreensão dedicados por minha noiva, Cinthia Sayuri, que nunca deixou de me apoiar nos momentos mais difíceis, com todo o meu amor, agradeço profundamente.

Sem o apoio financeiro da Fapesp, através da bolsa que recebi e através da verba para aquisição de material, este trabalho não teria sido possível. Agradeço à agência pelo suporte durante toda a duração do trabalho. O CNPq e a Capes também ofereceram apoio na forma de verba para aquisição de material e sou grato também a estas agências de fomento. 
Agradeço pela disponibilização dos recursos e pelo suporte do LCCA.

Outras pessoas provavelmente foram de grande importância no desenvolvimento deste trabalho, mas no entanto, pelo fato de que eu, como todos, sou suscetível a falhas, não me lembrarei de todos, mas mesmo assim serei sempre grato. 


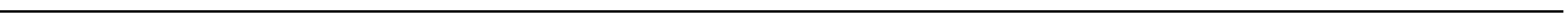




\section{RESUMO}

Nanotubos de carbono e polímeros condutores são fortes candidatos à miniaturização dos componentes eletrônicos disponíveis atualmente. Estudos teóricos afirmaram que 1/3 dos nanotubos seriam metálicos, enquanto que os outros seriam semicondutores, mas alguns grupos reportaram medidas experimentais evidenciando um pequeno gap eletrônico em tubos considerados metálicos. Protótipos de transístores compostos de nanotubos e moléculas orgânicas conjugadas foram propostos e foi observado que o recobrimento dos tubos por moléculas de pentaceno tornava os dispostivos menos suscetíveis à deposição de impurezas, o que diminuía a histerese na curva característica $i \times V$, ao mesmo tempo que a formação de cristais de pentaceno era favorecida.

Neste trabalho estudamos a estrutura eletrônica dos nanotubos $(5,5)$ e $(9,0)$ através de DFT e observamos presença de um gap nesses sistemas, assim como uma deformação de suas estruturas de ligações químicas, evidenciando a distorção de Peierls. O efeito do termo de troca de HartreeFock introduzido no funcional B3LYP foi avaliado variando-se seu peso e observando as propriedades destes sistemas. Em uma segunda etapa, utilizamos mecânica molecular e dinâmica molecular clássica com o campo de forças CVFF 950 e observamos a formação de estruturas de pentaceno em volta dos tubos, evidenciando o favorecimento da formação de cristais do mesmo quando depositado sobre os nanotubos. 


\section{ABSTRACT}

Carbon Nanotubes and conducting polymers are strong candidates for use in nanoscale electronic devices. Theoretical studies claimed that $1 / 3$ of the nanotubes are metallic, while the others are semiconductors, but some groups have reported experimental measurements of a small electronic gap in tubes considered metallic. Prototype transistors made of nanotubes and organic conjugated molecules were proposed and it has been noticed that the coverage of the tubes by pentacene molecules made those trasistors less susceptible to impurity deposition, reducing the hysteresis in the characteristic $i \times V$ curve, while the formation of pentacene cristals was favored.

In this work, we studied the electronic structure of the nanotubes $(5,5)$ and $(9,0)$ using DFT and noticed an electronic gap in those systems, as well as a deformation of their structures, similar to a Peierls distortion. The effect of the Hartree-Fock exchange included in the B3LYP functional was studied, as we varied its weight to obtain some properties of those systems. Later, we used molecular mechanics and classical molecular dynamics with the CVFF 950 force field and obtained structures compatible with pentacene crystals around the tubes, showing that the tubes in fact favor the formation of of these structures around them. 



\section{Índice}

1. Introdução 1

2. Compostos de carbono 5

2.1. Hibridização . . . . . . . . . . . . . . . . . . . 5

2.1.1. Hibridização $s p \ldots \ldots \ldots$. . . . . . . . . . . 6

2.1.2. Hibridização $s p^{2} \ldots \ldots \ldots \ldots \ldots$. . . . . . . . . . 7

2.1.3. Hibridização $s p^{3} \ldots \ldots \ldots$. . . . . . . . . . . 7

2.2. Compostos conjugados ..................... 8

2.3. Nanotubos de carbono . . . . . . . . . . . . . . . . 10

2.3.1. Estrutura . . . . . . . . . . . . . . . 10

2.3.2. Estrutura eletrônica . . . . . . . . . . . . . . 13

3. Distorção de Peierls 19

3.1. Distorção da cadeia 1D . . . . . . . . . . . . . . . . . . . 19

3.2. Surgimento do gap . . . . . . . . . . . . . . . . . . 24

$\begin{array}{lr}\text { 4. Metodologia } & 29\end{array}$

4.1. Mecânica Quântica - DFT . . . . . . . . . . . . . 29

4.2. Mecânica Molecular . . . . . . . . . . . . . . . 33 
5. Resultados - DFT 35

5.1. Poliacetileno . . . . . . . . . . . . . . . . . 35

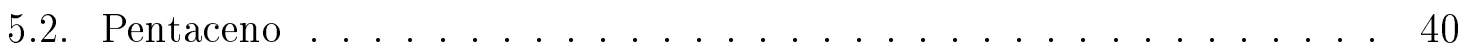

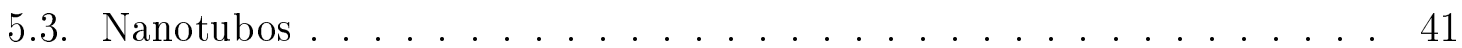

6. Resultados Clássicos 51

6.1. Mecânica Molecular . . . . . . . . . . . . . . . . 51

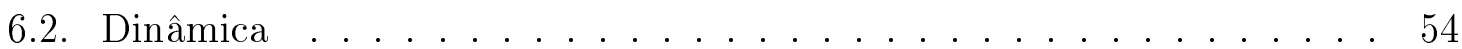

$\begin{array}{ll}\text { 7. Conclusões } & 57\end{array}$

$\begin{array}{ll}\text { Apêndices } & 59\end{array}$

A. Aproximação de Born-Oppenheimer 61

B. Teoria do Funcional da Densidade 65

B.1. Teoremas de Hohenberg e Kohn . . . . . . . . . . . . . . . 65

B.2. Equações de Kohn-Sham . . . . . . . . . . . . . . . . . . 67

B.3. Funcionais da DFT . . . . . . . . . . . . . . 71

$\begin{array}{ll}\text { C. Condições periódicas de contorno } & 73\end{array}$

C.1. Tight-Binding . . . . . . . . . . . . . . . 74

C.2. DFT híbrido com condições periódicas . . . . . . . . . . . . 75

$\begin{array}{ll}\text { D. Métodos clássicos } & 79\end{array}$

D.1. Campos de Forças . . . . . . . . . . . . . . . . . 79

D.2. Minimização de energia . . . . . . . . . . . . . . . . 82

D.3. Dinâmica . . . . . . . . . . . . . . . . 83

$\begin{array}{ll}\text { Referências Bibliográficas } & 85\end{array}$ 


\section{Introdução}

Após a observação dos primeiros nanotubos de carbono - estruturas formadas por átomos de carbono apenas, que apresentam diâmetros da ordem de nanômetros e comprimentos algumas ordens de grandeza maiores - por Iijima em 1991 [1], diversos

protótipos de dispositivos baseados em nanotubos foram propostos, [2-8] baseando-se em grande parte no fato que tais nanotubos poderiam ser metálicos ou semicondutores, de acordo com a configuração dos átomos constituintes dos mesmos. [9, 10]

Estudos posteriores mostraram que alguns tubos que eram considerados metálicos apresentavam um gap mensurável, e as possíveis causas para este fenômeno foram diversas, desde quebra de simetria para a estrutura dos tubos quando agrupados em feixes, [11-13] curvatura da estrutura dos tubos em relação à estrutura plana do grafeno [12-14] até o fenômeno conhecido como distorção de Peierls [15-18]. Esta última alternativa deve-se a um famoso teorema formulado por R. E. Peierls sobre sistemas unidimensionais que se acreditava serem condutores. [19]

Paralelamente a estas descobertas sobre os nanotubos, no final do século passado, os primeiro transístores orgânicos transparentes e flexíveis foram construídos depositando-se uma camada de composto orgânico semicondutor sobre um substrato plástico. Os eletrodos de fonte, dreno e porta utilizados, que no princípio eram feitos de ouro, foram substituídos por polímeros condutores, resultando em dispositivos flexíveis com performance próxima àquela dos dispositivos de silício contemporâneos. [20] Entre estes dispositivos, foram confeccionados transístores em que o meio semicondu- 
tor é composto de nanotubos de carbono depositados em uma superfície isolante. Tais transístores alcançaram performances também comparáveis aos dispositivos de silício usuais [21]. No entanto, os mesmos apresentaram histerese em suas curvas características $i \times V$, o que tornou inviável sua aplicação imediada, devido a impurezas aderidas às paredes dos tubos (água, ar e carbono resultante da síntese) [22]. Foi mostrado que a histerese é fortemente suprimida (figura 1.1) [21] ao se depositar uma camada de pentaceno, uma molécula conjugada formada por 5 anéis de benzeno enfileirados, (figura 1.2) sobre o dispositivo.

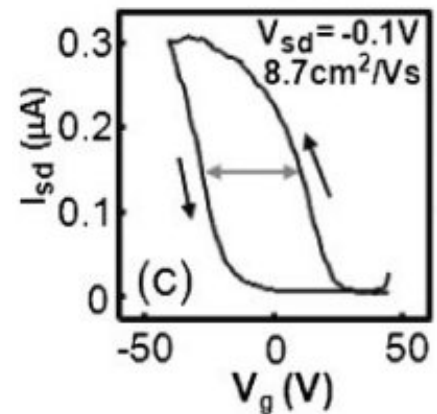

(a)

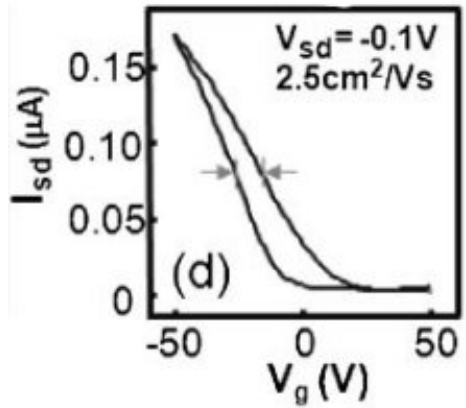

(b)

Figura 1.1.: Curva de $i \times V$ para um transístor de SWNTs antes (a) e após (b) a deposição de uma camada de pentaceno [21]<smiles>c1ccc2cc3cc4cc5ccccc5cc4cc3cc2c1</smiles>

Figura 1.2.: Estrutura da molécula de pentaceno

Os nanotubos também foram utilizados como eletrodos em outros transístores, e observou-se que além da mobilidade de portadores de carga aumentar em relação aos dispositivos com eletrodos metálicos, ao se utilizar o pentaceno como meio semicondutor, ocorria a formação de cristais da molécula nas vizinhanças dos eletrodos, (figura 
1.3) [23] sugerindo que os nanotubos auxiliam na formação de estruturas organizadas de pentaceno. [23] Uma explicação possível para tais fenômenos é a transferência de carga entre os nanotubos e o pentaceno, pelo fato de ambos possuirem estruturas conjugadas, capazes de interagir através de seus orbitais $\pi$.

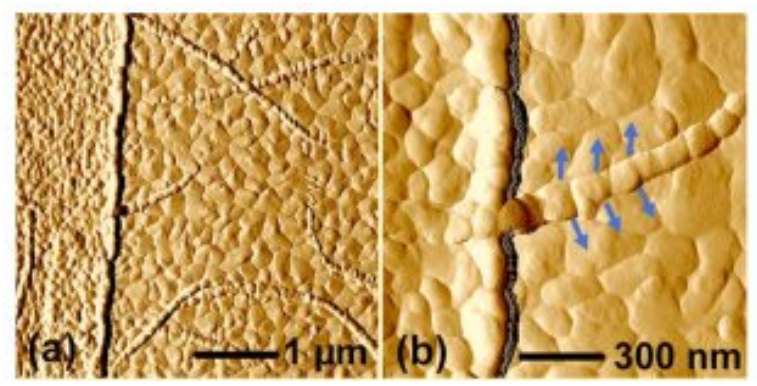

Figura 1.3.: Imagem obtida com microscópio de força atômica de filmes de pentaceno, depositados a temperatura ambiente, recobrindo eletrodos de nanotubo sobre um substrato de ouro (a) e de $\mathrm{SiO}_{2}$ tratado com $n$-octadecil trietoxisilano (b). As setas em (b) indicam uma possível direção da corrente.

Esta dissertação apresenta como principais objetivos a elucidação do comportamento condutor ou semicondutor de nanotubos de carbono de paredes únicas isolados e o estudo da formação de aglomerados de pentaceno ao redor de nanotubos do mesmo tipo. Para tanto, inicia-se com um capítulo sobre compostos de carbono, (capítulo 2) no qual são introduzidos os sistemas conjugados e os nanotubos de carbono. O fenômeno da distorção de Peierls para sistemas unidimensionais é exemplificado no capítulo 3 para uma cadeia e em seguida, encontra-se o capítulo 4 em que a metodologia utilizada neste trabalho, é apresentada, com a descrição dos métodos (maiores detalhes encontram-se nos apêndices). Finalmente os resultados são organizados nos capítulos 5 e 6 de acordo com os sistemas investigados e a metodologia utilizada, e são apresentadas as conclusões no capítulo final. 



\section{Compostos de carbono}

O carbono é um elemento químico com propriedades únicas. Por possuir 4 elétrons em sua camada de valência, na configuração $1 s^{2} 2 s^{2} 2 p^{2}$, cada átomo de carbono pode se ligar covalentemente a até outros 4 átomos, fazendo com que os compostos de carbono se apresentem em uma diversidade incrível de formas. Uma grande parte destes compostos é formada basicamente de carbono e hidrogênio, e são conhecidos como compostos orgânicos.

Pelo fato da energia do orbital $2 s$ e das energias dos orbitais $2 p\left(2 p_{x}, 2 p_{y}\right.$ e $\left.2 p_{z}\right)$ do carbono serem muito próximas, entre outros motivos, de acordo com um modelo proposto, um processo denominado hibridização destes orbitais poderia explicar certos detalhes das ligações covalentes observadas nos compostos.[24]

\subsection{Hibridização}

A hibridização dos orbitais atômicos foi proposta por Pauling em 1931 [24] como um modelo capaz de explicar a natureza das ligações químicas de alguns elementos, entre eles o carbono, nitrogênio e oxigênio. Tais elementos não apresentavam um número fixo de ligações covalentes, como esperado, baseado no número de elétrons desemparelhados na sua camada de valência. O modelo de Pauling então dizia que para que as ligações fossem possíveis, deveria haver uma combinação do orbital $2 s$ (que possui dois elétrons emparelhados) com os orbitais $2 p$, de modo que novos orbitais, denominados hibridizados pudessem fazer as ligações observadas. Deste modo, a hibridização 
poderia se dar de diversas formas, entre as de maior relevância para este trabalho, as hibridizações $s p, s p^{2}$ e $s p^{3}$, por serem as únicas que combinam apenas orbitais $s$ e $p$.

\subsubsection{Hibridização $s p$}

A hibridização $s p$ corresponde à formação de dois orbitais atômicos através da combinação linear do orbital $2 s$ e de um dos orbitais $2 p$ do átomo de carbono, de acordo com as equações 2.1. [9]

$$
\begin{aligned}
& \left|s p_{a}\right\rangle=\frac{1}{\sqrt{2}}\left(|2 s\rangle+\left|2 p_{x}\right\rangle\right) \\
& \left|s p_{b}\right\rangle=\frac{1}{\sqrt{2}}\left(|2 s\rangle-\left|2 p_{x}\right\rangle\right)
\end{aligned}
$$

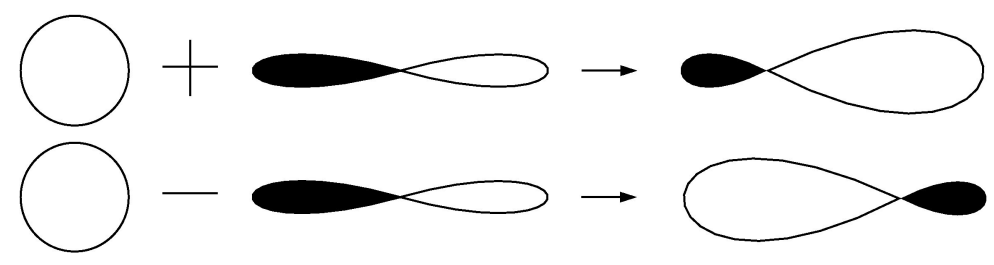

Figura 2.1.: Esquema ilustrando a formação dos orbitais híbridos $s p_{a}$ e $s p_{b}$ (equações 2.1)

Nessa configuração, os orbitais $s p_{a}$ e $s p_{b}$ encontram-se direcionados ao longo do eixo $x$, sendo capazes de participar de ligações do tipo $\sigma$. Os orbitais $2 p_{y}$ e $2 p_{z}$ não sofrem alterações, podendo formar ligações do tipo $\pi$ com átomos vizinhos, desse modo formando uma ligação tripla, como ocorre no etino ou acetileno $(H C \equiv C H)$. 


\subsubsection{Hibridização $s p^{2}$}

Neste caso, a combinação linear de um orbital $2 s$ e dois orbitais $2 p$ dá origem a 3 orbitais novos, conforme as equações 2.2. [9]

$$
\begin{aligned}
& \left|s p_{a}^{2}\right\rangle=\frac{1}{\sqrt{3}}|2 s\rangle+\sqrt{\frac{2}{3}}\left|2 p_{y}\right\rangle \\
& \left|s p_{b}^{2}\right\rangle=\frac{1}{\sqrt{3}}|2 s\rangle+\frac{1}{\sqrt{2}}\left|2 p_{x}\right\rangle-\frac{1}{\sqrt{6}}\left|2 p_{y}\right\rangle \\
& \left|s p_{c}^{2}\right\rangle=\frac{1}{\sqrt{3}}|2 s\rangle-\frac{1}{\sqrt{2}}\left|2 p_{x}\right\rangle-\frac{1}{\sqrt{6}}\left|2 p_{y}\right\rangle
\end{aligned}
$$

A escolha dos coeficientes para os orbitais $2 p_{x}$ e $2 p_{y}$ nestas equações é feita levando-se em consideração tanto a geometria de certos compostos orgânicos, como por exemplo o poliacetileno (figura 2.2), que apresenta um ângulo de $\approx 120^{\circ}$ entre duas ligações vizinhas quanto o fato de que estes orbitais devem ser ortogonais entre si.

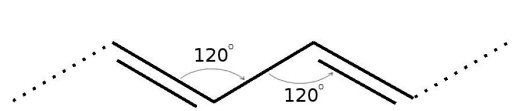

(a)

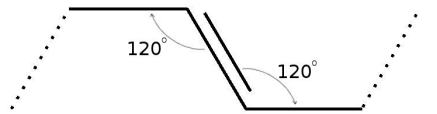

(b)

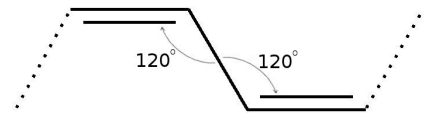

(c)

Figura 2.2.: poliacetileno na forma trans (a), trans-cisóide (b) e cis-transóide (c)

\subsubsection{Hibridização $s p^{3}$}

Para explicar o fato de que o carbono pode formar 4 ligações do tipo $\sigma$, foi proposto um outro tipo de hibridização, onde seus 3 orbitais $2 p$ combinam-se com o orbital $2 s$, formando 4 novos orbitais, dados pelas equações 2.3. [9] Novamente, o argumento utilizado para tal combinação é a geometria de estruturas com esse número de ligações, como por exemplo o diamante e o metano $\left(\mathrm{CH}_{4}\right)$ (figura 2.3), nos quais cada átomo 
de carbono faz 4 ligações com seus vizinhos, com a geometria de um tetraedro.

$$
\begin{aligned}
\left|s p_{a}^{3}\right\rangle & =\frac{1}{2}\left(|2 s\rangle+\left|2 p_{x}\right\rangle+\left|2 p_{y}\right\rangle+\left|2 p_{z}\right\rangle\right) \\
\left|s p_{b}^{3}\right\rangle & =\frac{1}{2}\left(|2 s\rangle-\left|2 p_{x}\right\rangle-\left|2 p_{y}\right\rangle+\left|2 p_{z}\right\rangle\right) \\
\left|s p_{c}^{3}\right\rangle & =\frac{1}{2}\left(|2 s\rangle-\left|2 p_{x}\right\rangle+\left|2 p_{y}\right\rangle-\left|2 p_{z}\right\rangle\right) \\
\left|s p_{d}^{3}\right\rangle & =\frac{1}{2}\left(|2 s\rangle+\left|2 p_{x}\right\rangle-\left|2 p_{y}\right\rangle-\left|2 p_{z}\right\rangle\right)
\end{aligned}
$$

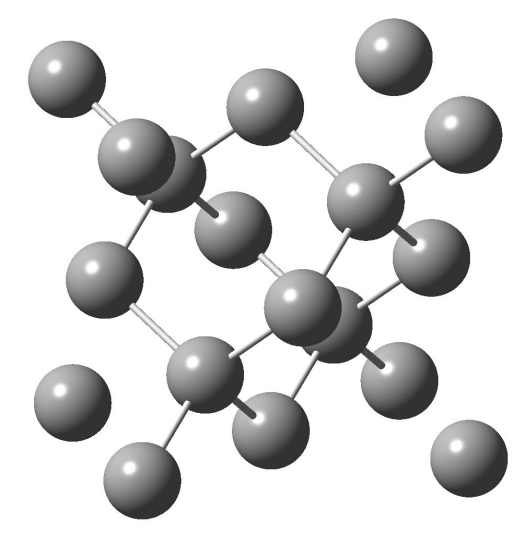

(a)

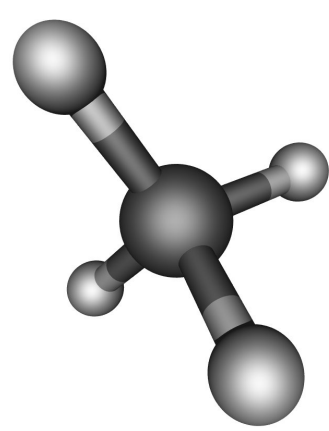

(b)

Figura 2.3.: Carbono na forma de diamante (a) e metano (b).

\subsection{Compostos conjugados}

Entre os compostos orgânicos, existe uma classe de moléculas que apresentam propriedades únicas, devidas à presença de um elétron em um orbital do tipo $p$ ao lado de uma ligação dupla, o que promove a deslocalização da nuvem eletrônica, dando origem a orbitais moleculares do tipo $\pi$ multicêntricos. Tais compostos são conhecidos como sistemas ou moléculas conjugadas.

Exemplos de sistemas conjugados são o poliacetileno (figura 2.2), o pentaceno (figura 1.2) e o grafeno (figura 2.4). Todos estes sistemas apresentam hibridização $s p^{2}$, fazendo com que haja um orbital $2 p_{z}$ não hibridizado por átomo de carbono, gerando assim a 
deslocalização eletrônica característica destes sistemas.<smiles></smiles>

Figura 2.4.: representação da estrutura bidimensional do grafeno

Dentre estes sistemas, um que merece especial atenção é o grafeno. O grafeno é uma estrutura bidimensional periódica formada de átomos de carbono com hibridização $s p^{2}$ (figura 2.4). Ao empilhar as lâminas de grafeno umas sobre as outras, a interação predominante é a de van der Waals, responsável por manter a estrutura coesa, dando origem ao grafite (figura 2.5). Outros sistemas, como os nanotubos de carbono ou os fulerenos podem ter sua estrutura exemplificada pelo dobramento de uma folha de grafeno, em formato cilíndrico no caso dos primeiros e formando uma estrutura aproximadamente esférica no caso dos últimos (figura 2.5). Note que tais estruturas não são formadas deste modo, sendo o dobramento apenas um artifício ilustrativo. Os nanotubos por exemplo, podem ser sintetizados em um ambiente totalmente desprovido de quaisquer estruturas de grafeno, utilizando-se apenas uma atmosfera de $C O$ puro mantida entre $700^{\circ} \mathrm{C}$ e $950^{\circ} \mathrm{C}$, na qual é bombeado um catalisador composto de partículas de cobalto e molibdênio, no processo conhecido como CoMoCat. [25] Os fulerenos devem apresentar alguns pentágonos no motivo de ligações entre os átomos de carbono para que sua estrutura fechada seja estável, os quais não estão presentes na estrutura de uma folha de grafeno [26]. 


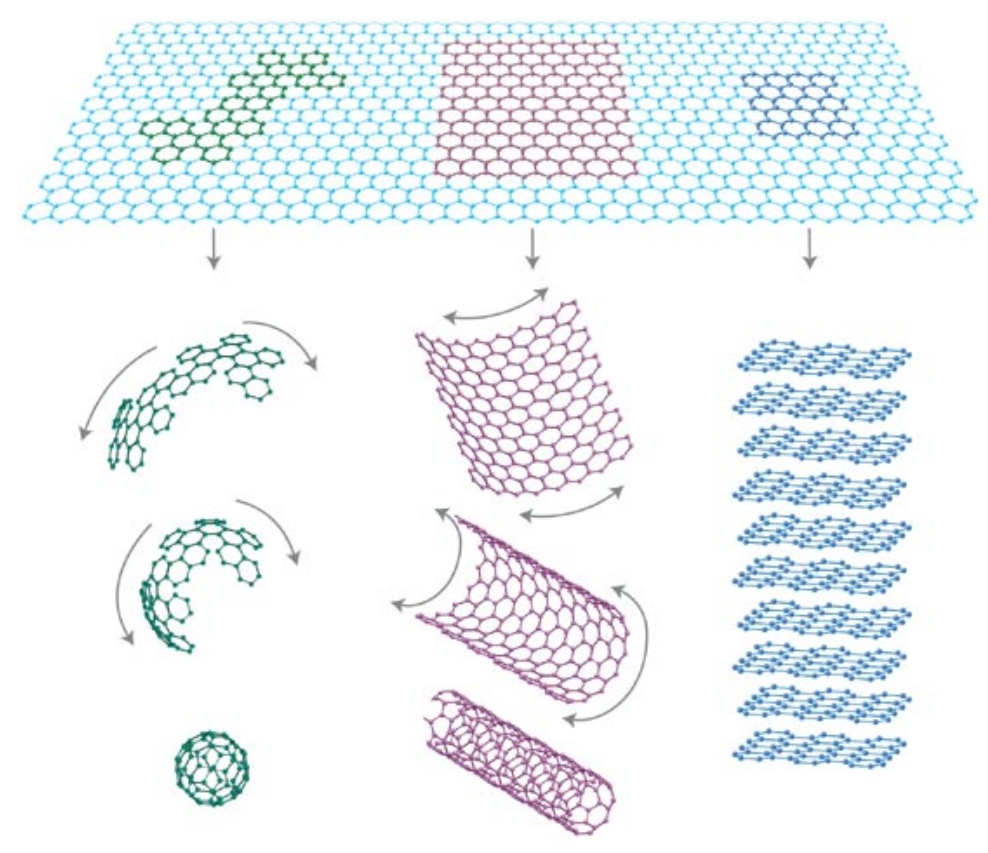

Figura 2.5.: Folha de grafeno ilustrando a estrutura de um fulereno (0-dimensional), um nanotubo (1-dimensional) e do grafite (3-dimensional) [27]

\subsection{Nanotubos de carbono}

Observados pela primeira vez por Iijima em 1991 [1], os nanotubos de carbono (CNTs - Carbon Nanotubes) são estruturas conjugadas cuja estrutura é exemplificada pelo enrolamento em formato cilíndrico de uma ou mais folhas de grafeno, denominados nanotubos de parede única (SWNTs - Single-Walled) ou nanotubos de paredes múltiplas (MWNTs - Multi-Walled), respectivamente ${ }^{1}$. Tais estruturas podem apresentar comprimentos de até algumas ordens de grandeza maiores do que seu diâmetro, sendo considerados sistemas quase unidimensionais.

\subsubsection{Estrutura}

Os nanotubos de parede única são descritos por dois índices $(n, m)$, com $n$ e $m$ inteiros (e $0 \leq m \leq n$ pela simetria da rede) que determinam o vetor quiral $\vec{C}_{h}$

\footnotetext{
${ }^{1}$ Note novamente que os CNTs não são formados enrolando-se folhas de grafeno!
} 
ilustrado na figura 2.6, de acordo com a equação 2.4, onde $\overrightarrow{a_{1}}$ e $\overrightarrow{a_{2}}$ são os vetores primitivos da rede hexagonal do grafeno [28]. Ao sobrepor os átomos de carbono que se encontram no início e no final deste vetor, como se enrolássemos a estrutura formada pelos hexágonos em forma de cilindro, obtém-se a estrutura de um nanotubo de carbono. O vetor de translação $\vec{T}$ determina uma célula unitária do nanotubo [28], é perpendicular a $\overrightarrow{C_{h}}$ e paralelo ao eixo do tubo.

$$
\overrightarrow{C_{h}}=n \overrightarrow{a_{1}}+m \overrightarrow{a_{2}}
$$

O diâmetro de um nanotubo de parede simples é dado através da relação entre o mesmo e o módulo do vetor quiral $\overrightarrow{C_{h}}$ em 2.5

$$
d=\frac{\left|\overrightarrow{C_{h}}\right|}{\pi}=\frac{\sqrt{n^{2}\left|\overrightarrow{a_{1}}\right|^{2}+m^{2}\left|\overrightarrow{a_{2}}\right|^{2}+2 m n \overrightarrow{a_{1}} \cdot \overrightarrow{a_{2}}}}{\pi}=\frac{a}{\pi} \sqrt{n^{2}+m^{2}+m n}
$$

onde foi utilizado o fato que $\left|\overrightarrow{a_{1}}\right|=\left|\overrightarrow{a_{2}}\right|=a$ e $\overrightarrow{a_{1}} \cdot \overrightarrow{a_{2}}=(1 / 2) a^{2}$, em que $a=1,44 \sqrt{3} \AA=$ 2, 49 A é o parâmetro da rede direta do grafeno.

O ângulo quiral $\theta$ (figura 2.6) é dado por

$$
\cos \theta=\frac{\overrightarrow{C_{h}} \cdot \overrightarrow{a_{1}}}{\left|\overrightarrow{C_{h}}\right|\left|\overrightarrow{a_{1}}\right|}=\frac{2 n+m}{2 \sqrt{n^{2}+m^{2}+n m}}
$$

Os nanotubos podem ser classificados de acordo com seus índices $(n, m)$ da seguinte maneira:

- Se $n=m$, o tubo é do tipo armchair. O nome é proveniente do motivo formado pelas ligações em sua seção reta (figura 2.7). Tal tipo de tubo apresenta um ângulo quiral $\theta=30^{\circ}$

- Se $m=0$, o tubo é do tipo zigzag, com explicação similar para o nome. O ângulo quiral neste caso é $\theta=0^{\circ}$.

- O tubo é do tipo quiral para os outros casos, em que o ângulo quiral encontra-se 
no intervalo $0^{\circ} \leq \theta \leq 30^{\circ}$.

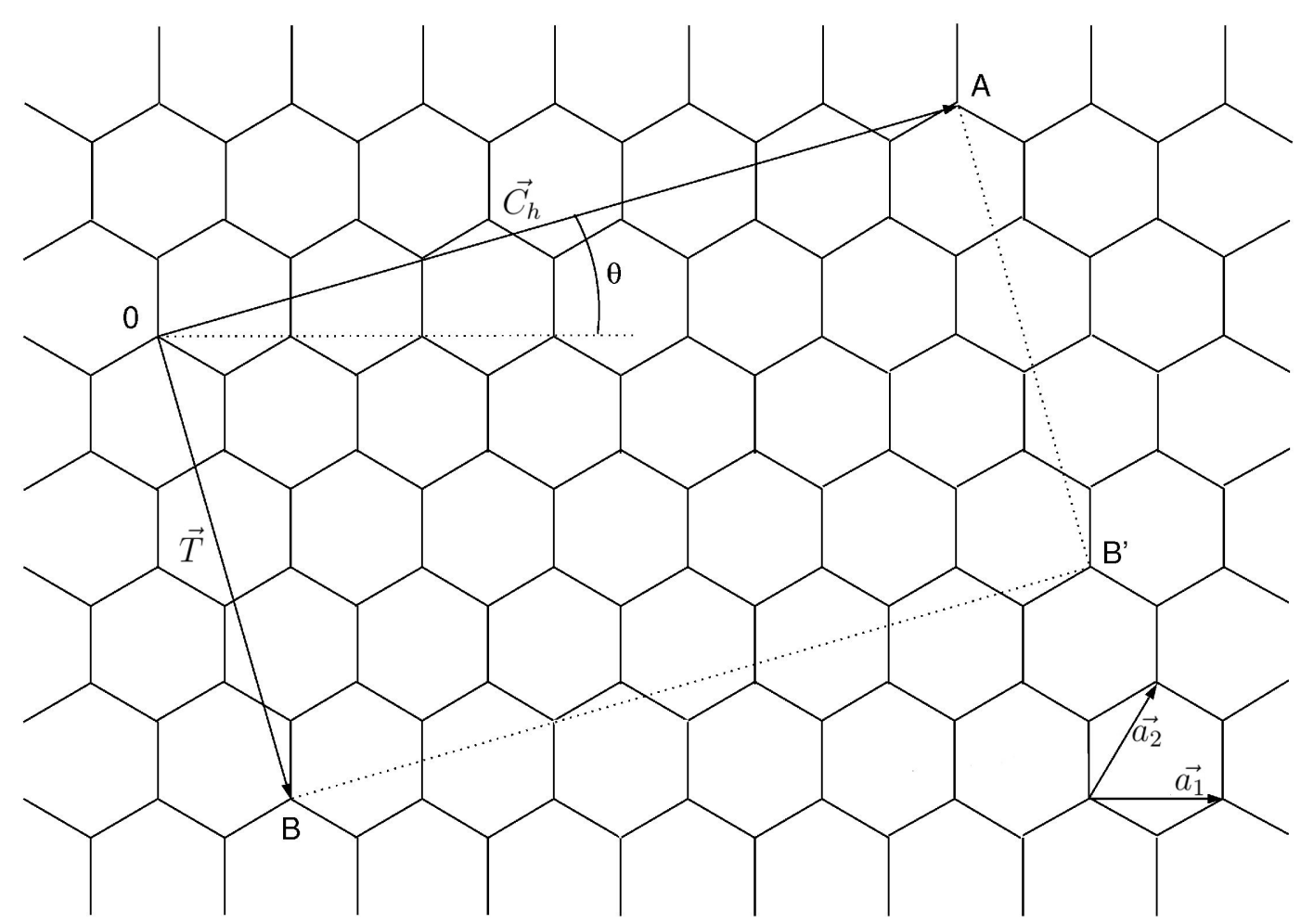

Figura 2.6.: A célula unitária de um SWNT desenrolada é determinada pelos pontos $O, A, B$ e $B^{\prime}$. Ao se enrolar a folha com os hexágonos de modo que os pontos $O$ e $A$ coincidam, ao mesmo tempo que os pontos $B$ e $B^{\prime}$ também coincidam, forma-se um SWNT, neste caso, um tubo $(5,2)$. Os vetores $\overrightarrow{a_{1}}$ e $\overrightarrow{a_{2}}$ são os vetores unitários da rede do grafeno [28]

O vetor de translação $\vec{T}$ é definido pelos índices inteiros $\left(t_{1}, t_{2}\right)$, através de

$$
\vec{T}=t_{1} \overrightarrow{a_{1}}+t_{2} \overrightarrow{a_{2}}
$$

e com o fato de que $\overrightarrow{C_{h}} \cdot \vec{T}=0$, pode-se obter $t_{1}$ e $t_{2}$ em função de $n$ e $m$, através das equações 2.4 e 2.7

$$
t_{1}=\frac{2 m+n}{d_{R}} \quad t_{2}=-\frac{2 n+m}{d_{R}}
$$

com

$$
d_{R}= \begin{cases}d & , \text { se } n-m \text { não é um múltiplo de } 3 \\ 3 d & , \text { se } n-m \text { é um múltiplo de } 3\end{cases}
$$




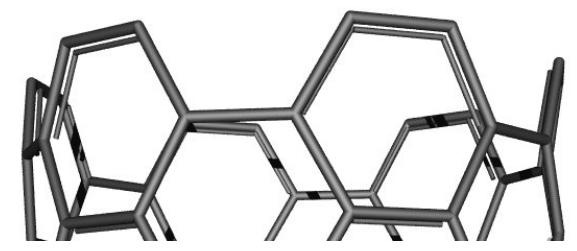

(a)

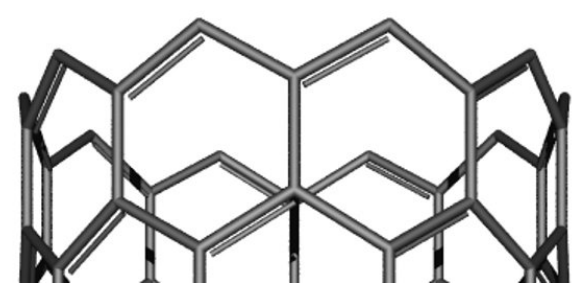

(b)

Figura 2.7.: Seção transversal dos nanotubos do tipo armchair (a) e zigzag (b).

\subsubsection{Estrutura eletrônica}

A estrutura eletrônica de um SWNT pode ser obtida a partir da estrutura eletrônica do grafeno. Utilizando-se o modelo tight-binding[9][28] (apêndice C.1) de primeiros vizinhos, os elementos de matriz da hamiltoniana e da matriz de overlap para a configuração ilustrada na figura 2.8 são

$$
\begin{aligned}
& H_{A A}=\left\langle\phi_{A}(\vec{r})|H| \phi_{A}(\vec{r})\right\rangle=H_{B B}=\left\langle\phi_{B}(\vec{r})|H| \phi_{B}(\vec{r})\right\rangle=\epsilon \\
& H_{A B}=\sum_{i} e^{i \vec{k} \cdot \vec{R}_{i}}\left\langle\phi_{A}(\vec{r})|H| \phi_{B}\left(\vec{r}-\vec{R}_{i}\right)\right\rangle=\beta\left(e^{i \vec{k} \cdot \vec{R}_{1}}+e^{i \vec{k} \cdot \vec{R}_{2}}+e^{i \vec{k} \cdot \vec{R}_{3}}\right)=\beta f(\vec{k}) \\
& S_{A A}=\left\langle\phi_{A}(\vec{r}) \mid \phi_{A}(\vec{r})\right\rangle=S_{B B}=\left\langle\phi_{B}(\vec{r}) \mid \phi_{B}(\vec{r})\right\rangle=1 \\
& S_{A B}=\sum_{i} e^{i \vec{k} \cdot \vec{R}_{i}}\left\langle\phi_{A}(\vec{r}) \mid \phi_{B}\left(\vec{r}-\vec{R}_{i}\right)\right\rangle=\sigma f(\vec{k})
\end{aligned}
$$

com

$$
f(\vec{k})=e^{i k_{x} a / \sqrt{3}}+2 e^{-i k_{x} a / 2 \sqrt{3}} \cos \left(\frac{k_{y} a}{2}\right)
$$

em que foram utilizadas as coordenadas dos vetores da figura 2.8.

De acordo com o apêndice C.1, a energia então é obtida através da resolução da equação secular,

$$
\left|\begin{array}{cc}
\epsilon-E & (\beta-E \sigma) f(\vec{k}) \\
(\beta-E \sigma) f^{*}(\vec{k}) & \epsilon-E
\end{array}\right|=0
$$




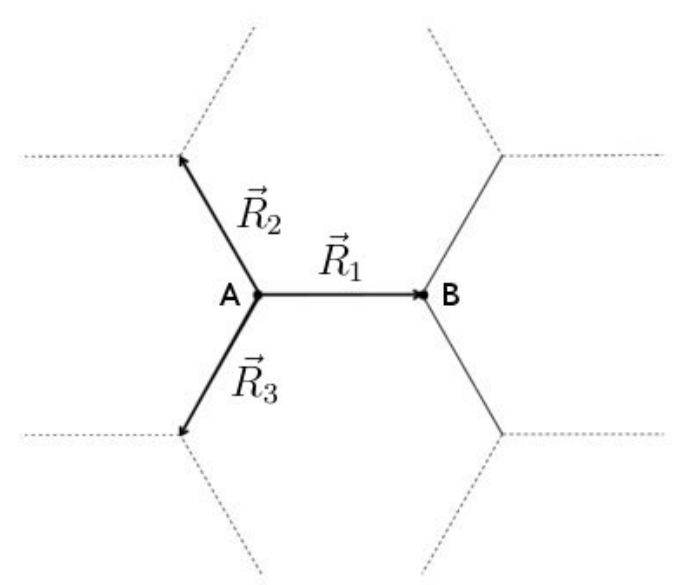

Figura 2.8.: Rede direta de uma lâmina de grafeno e seus dois átomos da célula unitária

A solução fornece as bandas de energia dos orbitais $\pi$ e $\pi^{*}$, que são respectivamente os orbitais ligante ${ }^{2}$ e antiligante ${ }^{3}$ do grafeno,

$$
E(\vec{k})=\frac{\epsilon \pm \beta \omega(\vec{k})}{1 \pm \sigma \omega(\vec{k})}
$$

onde $\epsilon$ é a energia do orbital $2 p$ do carbono ${ }^{4}$ e $\omega(\vec{k})$ é escrito como

$$
\omega(\vec{k})=\sqrt{|f(\vec{k})|^{2}}=\sqrt{1+4 \cos \left(\frac{\sqrt{3} k_{x} a}{2}\right) \cos \left(\frac{k_{y} a}{2}\right)+4 \cos ^{2}\left(\frac{k_{y} a}{2}\right)}
$$

A figura 2.9 mostra as bandas do grafeno (equação 2.11). Note que as bandas se tocam apenas nos limites da zona de Brillouin (pontos $K$ ), que tem a forma de um hexágono.

\footnotetext{
${ }^{2}$ solução com sinal +

${ }^{3}$ solução com sinal -

${ }^{4}$ Esta energia não é simplesmente a energia do orbital, pois deve-se levar em consideração o fato de haver um potencial periódico no hamiltoniano.
} 


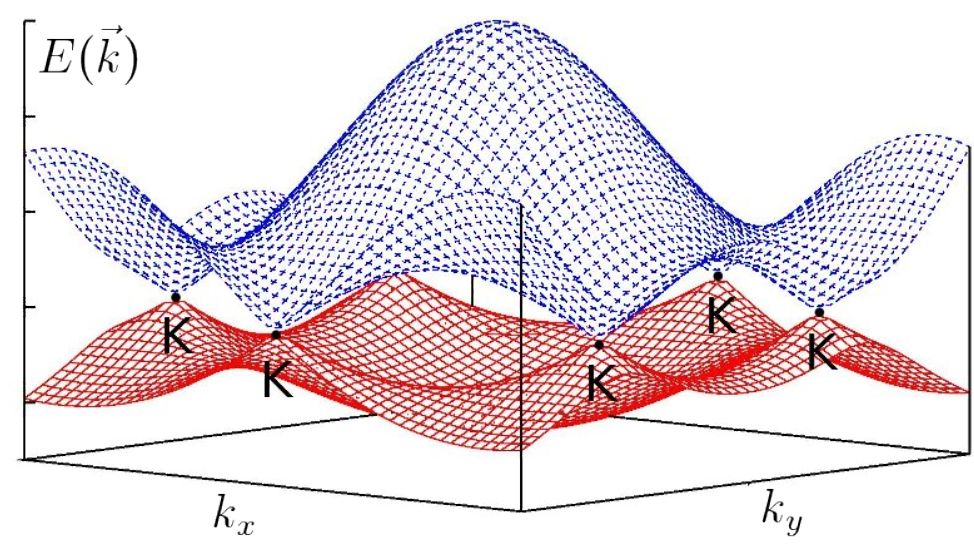

Figura 2.9.: As relações de dispersão das bandas $\pi$ e $\pi^{*}$ do grafeno plotadas sobre toda a extensão da zona de Brillouin[28].

Como os nanotubos possuem uma dimensão restrita ao longo do vetor $\vec{C}_{h}$, o vetor da rede recíproca associado a esta direção torna-se quantizado. Usando as relações

$$
\begin{array}{ll}
\overrightarrow{C_{h}} \cdot \vec{K}_{1}=2 \pi & \vec{T} \cdot \vec{K}_{1}=0 \\
\overrightarrow{C_{h}} \cdot \vec{K}_{2}=0 & \vec{T} \cdot \vec{K}_{2}=2 \pi
\end{array}
$$

obtém-se

$$
\overrightarrow{K_{1}}=\frac{1}{N}\left(-t_{2} \overrightarrow{b_{1}}+t_{1} \overrightarrow{b_{2}}\right) \quad \overrightarrow{K_{2}}=\frac{1}{N}\left(m \overrightarrow{b_{1}}-n \overrightarrow{b_{2}}\right)
$$

Note que $N \vec{K}_{1}$ é um vetor da rede recíproca do grafeno, e com isto, dois vetores que diferem por $N \vec{K}_{1}$ são equivalentes. Logo, os vetores $\mu \vec{K}_{1},(\operatorname{com} \mu=0,1, \cdots, N-1)$ não são vetores da rede recíproca do grafeno, dando origem a $N$ vetores discretos associados à direção $\overrightarrow{C_{h}}$, e cujo comprimento é a extensão da zona de Brillouin $2 \pi / T$, como ilustrado na figura 2.10. Com isto, obtemos um conjunto de $N$ relações de dispersão de energia que são seções da relação do grafeno. $N$ é o número de elétrons $\pi$ na célula unitária do tubo. 

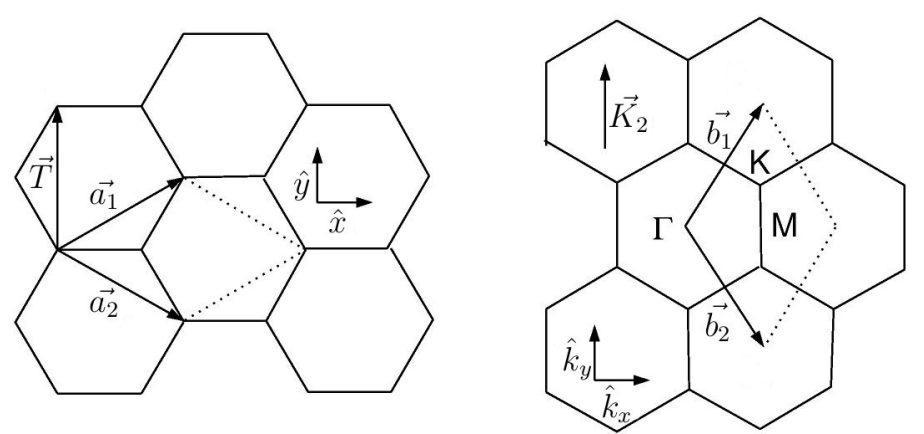

(a)
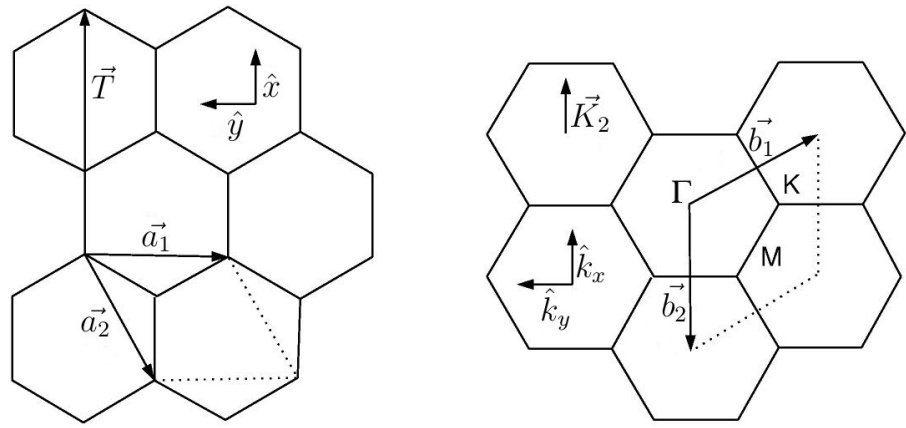

(b)

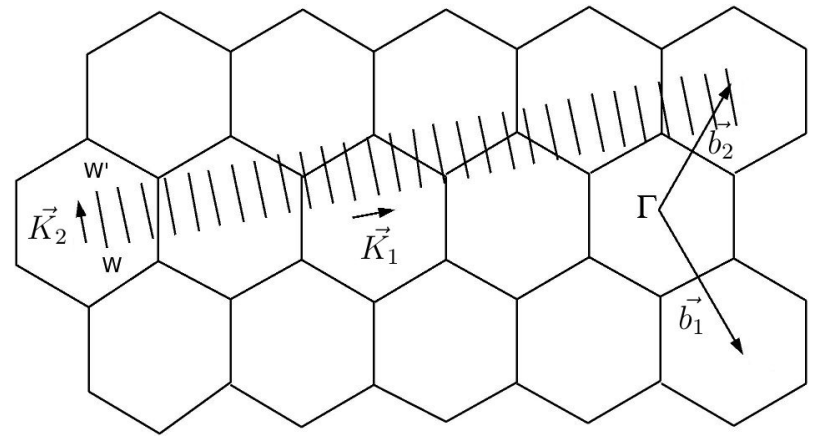

(c)

Figura 2.10.: Partes da rede direta e rede recíproca de um tubo armchair (a), zigzag (b) e a zona de Brillouin de um nanotubo representada pelo segmento $W W^{\prime}(\mathrm{c})$

A relação de dispersão para os nanotubos é dada pela mesma expressão obtida para o grafeno (equação 2.11), mas no entando, os vetores $\vec{k}$ são discretizados na direção $\vec{K}_{1}$, através do número $\mu$, resultando em

$$
E_{\mu}(\vec{k})=E\left(k \frac{\vec{K}_{2}}{\left|\vec{K}_{2}\right|}+\mu \overrightarrow{K_{1}}\right), \quad \mu=0, \cdots, N-1 \text { e }-\frac{\pi}{T}<k<\frac{\pi}{T}
$$


Os segmentos enfileirados da figura 2.10 (c) representam as linhas dadas por $k \vec{K}_{2} /\left|\vec{K}_{2}\right|+$ $\mu \vec{K}_{1}$. Se algum destes segmentos passa sobre um ponto $K$ da zona de Brillouin do grafeno (ponto onde as bandas $\pi$ e $\pi^{*}$ se encostam na figura 2.9), o tubo apresenta um comportamento condutor. É possível mostrar que para $n-m$ múltiplo de 3 esta condição é satisfeita,[9] levando ao fato de que 1/3 de todos os nanotubos são metálicos, enquanto que os outros são semicondutores.

No entanto, ao ter parte de sua banda de valência preenchida, os nanotubos metálicos deveriam apresentar uma instabilidade em sua rede direta. Isso levaria ao aumento da célula unitária e uma redução da mesma ordem em sua rede recíproca. O nanotubo $(5,5)$, por exemplo, apresenta $1 / 3$ da banda preenchida, fazendo com que esta instabilidade ocasione a triplicação do comprimento da célula unitária e a diminuição em um fator de 3 na rede recíproca. Tal mecanismo é conhecido como distorção de Peierls [9] e com isto ocorre uma supressão dos níveis com energia próxima à energia de Fermi do sistema, aparecendo um gap. Esta distorção ocorre a uma temperatura crítica conhecida como temperatura de transição de Peierls e é estimada como sendo da ordem de alguns Kelvins [18]. 



\section{Distorção de Peierls}

Em 1955, Rudolf E. Peierls em seu livro Quantum Theory of Solids [19] enunciou o seguinte teorema: "... para um metal unidimensional com a banda parcialmente preenchida, a estrutura de cadeia regularmente espaçada nunca será estável.". Tal afirmação significa basicamente que uma cadeia do tipo que está ilustrada na figura 3.1 não será estável se tiver a banda parcialmente preenchida. Distorções nesta cadeia, que alteram a distância entre átomos vizinhos, ocasionarão uma diminuição na energia eletrônica e um aumento na energia de repulsão entre os núcleos, de maneira que o saldo energético seja negativo, fazendo com que esta configuração distorcida seja energeticamente mais estável. Esta distorção ficou conhecida na literatura como distorção de Peierls [19].

Este assunto foi abordado durante a execução do projeto de mestrado, pois os nanotubos $(5,5)$ e $(9,0)$ se enquadravam nesta definição de metais unidimensionais (conforme seção 2.3).

\subsection{Distorção da cadeia 1D}

Para ilustrar tal distorção, um desenvolvimento análogo ao que foi feito por $S u, S c h$ rieffer e Heeger [29], no qual é verificada tal distorção em uma cadeia unidimensional de trans-poliacetileno, será reproduzido nesta seção.

Considere o sistema formado por uma cadeia unidimensional monoatômica de $N$ sítios, com comprimento $L=N a$ e 1 elétron de valência por átomo. 


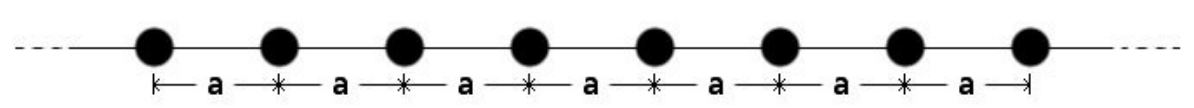

Figura 3.1.: Cadeia de $N$ íons igualmente espaçados de $a$.

Dentro da aproximação de Born-Oppenheimer (apêndice A), podemos separar este problema em duas partes: uma hamiltoniana que envolve apenas as coordenadas eletrônicas e outra hamiltoniana que descreve a dinâmica do caroço, correspondente ao núcleo em conjunto com seus elétrons mais internos, uma vez que a interação entre elétrons de caroço de um sítio com os elétrons de caroço dos sítios vizinhos pode ser desprezada . Considerando o núcleo do sítio $n$ mais seus elétrons internos como uma distribuição de carga esfericamente simétrica de carga $Z$, podemos escrever a energia potencial deste conjunto como sendo proveniente da interação com seus primeiros vizinhos.

$$
U_{n}=Z^{2} e^{2}\left(\frac{1}{\left|x_{n}-x_{n-1}\right|}+\frac{1}{\left|x_{n}-x_{n+1}\right|}\right)
$$

Suponha que os átomos da cadeia sofram pequenos deslocamentos dados por $u_{n}=$ $(-1)^{n} u$, como ilustrado na figura 3.2.

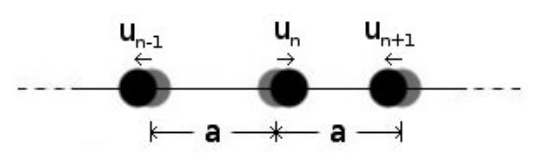

Figura 3.2.: Três sítios da cadeia distorcida para $n$ par $\left(u_{n}>0\right.$ e $\left.u_{n-1}, u_{n+1}<0\right)$.

As posições dos átomos podem ser escritas em termos das posições do sítios da rede e dos deslocamentos como $x_{n}=n a+u_{n}$. Logo, a energia potencial de um sítio pode 
ser expressa então como

$$
\begin{aligned}
U_{n}(u) & =Z^{2} e^{2}\left(\frac{1}{\left|a+(-1)^{n} u-(-1)^{n-1} u\right|}+\frac{1}{\left|a-(-1)^{n} u+(-1)^{n+1} u\right|}\right) \\
& =\frac{Z^{2} e^{2}}{a}\left(\frac{1}{\left|1+\frac{2(-1)^{n} u}{a}\right|}+\frac{1}{1-\frac{2(-1)^{n} u}{a} \mid}\right)
\end{aligned}
$$

Como os deslocamentos são muito pequenos em relação à distância entre os sítios, $(u \ll a)$, podemos expandir esta função ao redor de $u=0$, obtendo uma aproximação até a ordem de $u^{2}$,

$$
U_{n}(u)=\frac{8 Z^{2} e^{2}}{a^{3}} u^{2}=\frac{1}{2} K u^{2}
$$

onde o termo constante da expansão foi tomado como referência da energia e $K=$ $(4 Z e)^{2} / a^{3}$. Com isto, a energia potencial total de interação coulombiana entre os íons da cadeia é dada por

$$
U(u)=\sum_{n} U_{n}(u)=2 N K u^{2}
$$

A energia cinética do íon localizado no sítio $n$ é dada por

$$
T_{n}=\frac{1}{2} M \dot{x}_{n}^{2}=\frac{1}{2} M\left[n \dot{a}+\dot{u}_{n}\right]^{2}=\frac{1}{2} M \dot{u}_{n}^{2}
$$

$M$ é a massa do núcleo e elétrons de caroço no sítio.

Por último, a energia dos elétrons de valência, pode ser obtida através de um cálculo do tipo Tight-Binding (apêndice C.1) levando-se em consideração apenas as interações entre primeiros vizinhos na rede. Como a integral de hopping é proporcional ao overlap entre orbitais atômicos de sítios vizinhos, que por sua vez decai exponencialmente com a distância entre estes vizinhos, podemos expandi-la até $1^{a}$ ordem em função dos deslocamentos, obtendo [29] [30]

$$
\beta_{n+1, n}=\beta_{0}-\alpha\left(u_{n+1}-u_{n}\right)
$$

com $\beta_{0}$ sendo a integral de hopping da cadeia uniforme e $\alpha$ a constante de acoplamento 
elétron-fônon. Tal aproximação é válida desde que os deslocamentos $u_{n}$ sejam pequenos $[29]$.

Com isto, a hamiltoniana eletrônica nesta aproximação pode ser escrita como

$$
H=-\sum_{n, s}\left[\epsilon_{F} c_{n, s}^{\dagger} c_{n, s}+\beta_{n+1, n}\left(c_{n+1, s}^{\dagger} c_{n, s}+c_{n, s}^{\dagger} c_{n+1, s}\right)\right]+\sum_{n}\left[U_{n}(u)+T_{n}(\dot{u})\right]
$$

Nesta expressão, $\epsilon_{F}$ é a energia de Fermi e os operadores $c_{n, s}^{\dagger}$ e $c_{n, s}$ criam e destroem elétrons de valência no sítio $n \operatorname{com} \operatorname{spin} s$, respectivamente. Tais operadores obedecem as regras de anticomutação de férmions $\left\{a_{i}, a_{j}\right\}=\left\{a_{i}^{\dagger}, a_{j}^{\dagger}\right\}=0$ e $\left\{a_{i}, a_{j}^{\dagger}\right\}=\delta_{i j}$. Os operadores $u_{n}$ e $p_{n}=M \dot{u}_{n}$ satisfazem as relações canônicas de comutação, $\left[p_{i}, u_{j}\right]=$ $-i \hbar \delta_{i j}$

A interação entre os elétrons de valência neste modelo está parcialmente incluída utilizando-se valores ajustados para $\beta_{0}$ e $\alpha$, mas isso só é válido para o caso em que tal interação é fraca. [29]

Deste modo, a energia proveniente da parte eletrônica é obtida através da equação secular:

$$
\left|\begin{array}{cc}
\epsilon_{F}-E & \left(\beta_{0}+2 \alpha u\right) e^{i k a}+\left(\beta_{0}-2 \alpha u\right) e^{-i k a} \\
\left(\beta_{0}+2 \alpha u\right) e^{-i k a}+\left(\beta_{0}-2 \alpha u\right) e^{i k a} & \epsilon_{F}-E
\end{array}\right|=0
$$

Os autovalores são então

$$
E_{e l}(u, k)=\epsilon_{F} \pm \sqrt{\left(2 \beta_{0} \cos k a\right)^{2}+(4 \alpha u \operatorname{sen} k a)^{2}}
$$

E com isto, a energia total do sistema pode ser escrita como

$$
E(u)=-2 \sum_{k} E_{e l}(u, k)+2 N K u^{2}
$$

Pode-se notar que a soma em $n$ e $s$ na equação 3.7 foi substituída por uma soma em $k$ multiplicada por 2, pois para cada ponto $k$ no espaço recíproco, há dois elétrons com spins opostos. O termo da energia cinética dos íons em 3.7, que envolve a derivada 
dos deslocamentos $\left(\dot{u}_{n}\right)$ foi eliminado pois nesta situação $u$ é constante.

Trocando a soma em $k$ por uma integral sobre a primeira zona de Brillouin, e substituindo a expressão de $E_{e l}$ de 3.9 na equação 3.10 ,

$$
\begin{aligned}
E(u) & =-\frac{N a}{\pi} \int_{-\pi / 2 a}^{\pi / 2 a} d k\left[\epsilon_{F}-\sqrt{\left(2 \beta_{0} \cos k a\right)^{2}+(4 \alpha u \operatorname{sen} k a)^{2}}\right]+2 N K u^{2} \\
& =-N \epsilon_{F}+\frac{4 N\left|\beta_{0}\right|}{\pi} \int_{0}^{\pi / 2} d x \sqrt{1-\left(1-z^{2}\right) \operatorname{sen}^{2} x}+2 N K u^{2}
\end{aligned}
$$

Onde o sinal negativo da equação 3.9 foi escolhido pois corresponde à banda ocupada e $z=2 \alpha u / \beta_{0}$. A integral na expressão acima é conhecida como integral elíptica completa do $2^{\circ}$ tipo, e pode ser resolvida expandindo-se o integrando ao redor de $z=0 .[29][9]$

$$
E\left(1-z^{2}\right)=\int_{0}^{\pi / 2} d x \sqrt{1-\left(1-z^{2}\right) \operatorname{sen}^{2} x} \approx 1+\frac{1}{2}\left(\frac{\log 4}{|z|}-\frac{1}{2}\right) z^{2}
$$

Com este resultado, a energia total por sítio $(E(u) / N)$ pode ser expressa então como

$$
E(u)=-\epsilon_{F}+\frac{4 \beta_{0}}{\pi}\left[1+\frac{1}{4}\left(\frac{\left|\beta_{0}\right| \log 4}{\alpha|u|}-1\right)\left(\frac{2 \alpha}{\beta_{0}}\right)^{2} u^{2}\right]+2 K u^{2}
$$

É possível notar nesta expressão que o termo correspondente à energia elástica devida ao deslocamento dos núcleos é proporcional a $u^{2}$ enquanto que o termo correspondente à energia dos elétrons apresenta um termo proporcional a $-|u|\left(\beta_{0}<0\right)$, logo, é esperado que um pequeno deslocamento da cadeia gere uma configuração mais energeticamente estável. O gráfico desta função para $\epsilon_{F}=0 \mathrm{eV}, \beta_{0}=-2,5 \mathrm{eV}, \alpha=4.1$ $\mathrm{eV}$ e $K=21 \mathrm{eV} / \AA$, que são os valores obtidos na literatura para o trans-poliacetileno [29] encontra-se na figura 3.3.

Pode-se notar que há 3 configurações de equilíbrio para a cadeia, para os valores de $u= \pm u_{0}, 0$, com os dois primeiros valores para a distorção dando origem a configurações estáveis e com energia mais negativa do que a configuração da cadeia uniforme, que apresenta um equilíbrio instável. Com isso, é possível dizer que o estado de menor 


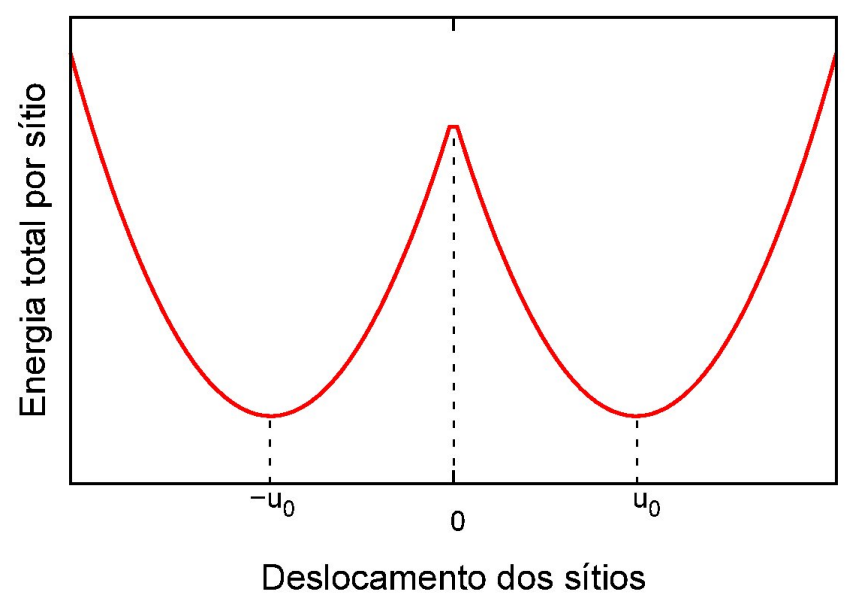

Figura 3.3.: Energia total por sítio em função do deslocamento $u$ na cadeia.

energia de uma cadeia deste tipo é o estado distorcido.

\subsection{Surgimento do gap}

Uma consequência desta distorção é o surgimento de um gap no limite da zona de Brillouin da estrutura distorcida, com a célula unitária aumentada $n$ vezes, quando a banda de valência está ocupada por uma fração $1 / n$ dos elétrons permitidos. Isto pode ser demonstrado escrevendo-se a hamiltoniana do sistema da cadeia ilustrada na figura 3.1 como

$$
H=H^{0}+V(x)
$$

onde $H^{0}$ é o hamiltoniano de um elétron livre $\left(p^{2} / 2 m\right)$ e $V(x)$ é um potencial periódico, de período $a$, ao qual os elétrons de valência desta cadeia estão submetidos.

Desprezando a interação entre os elétrons de valência e considerando o potencial como uma perturbação, temos que as funções de onda não perturbadas e os níveis de energia não perturbados são,

$$
\psi_{k}^{(0)}(x)=\frac{1}{\sqrt{L}} e^{-i k x} \quad E_{k}^{(0)}=\frac{\hbar^{2} k_{n}^{2}}{2 m}
$$


Para este sistema unidimensional, a superfície de Fermi é determinada pelos pontos $k=-\pi / a$ e $k=\pi / a$ e a correção de primeira ordem para a energia ${ }^{1}$ é dada por[31]:

$$
E_{k}^{(1)}=\left\langle\psi_{k}^{(0)}|V| \psi_{k^{\prime}}^{(0)}\right\rangle=\frac{1}{L} \int_{0}^{L} d x e^{i\left(k-k^{\prime}\right) x} V(x)=\hat{V}\left(k-k^{\prime}\right)
$$

Devido à periodicidade de $V(x)$, esta integral é não nula somente se o argumento da exponencial apresentar a mesma periodicidade do potencial ${ }^{2}$, ou seja, deve-se ter

$$
k=k^{\prime}+\frac{2 \pi}{a} l
$$

com $l$ inteiro.

Para $l=0$, de acordo com 3.16, temos

$$
E_{k=k^{\prime}}^{(1)}=\frac{1}{L} \int_{0}^{L} d x V(x)
$$

que é somente a média espacial do potencial. Tomando esse valor como referência da energia, ou seja, fazendo $E_{k=k^{\prime}}^{(1)}=0$, vemos que a correção da energia será dada pela segunda ordem em teoria de perturbação ${ }^{3}$,

$$
E_{k}^{(2)}=\sum_{k \neq k^{\prime}} \frac{\left|\left\langle\psi_{k}^{(0)}|V| \psi_{k^{\prime}}^{(0)}\right\rangle\right|^{2}}{E_{k}^{(0)}-E_{k^{\prime}}^{(0)}}
$$

A equação 3.19 no entanto apresenta um problema, pois, de acordo com 3.17 e 3.15 ,

$$
\begin{aligned}
E_{k}^{(0)}-E_{k^{\prime}}^{(0)} & =\frac{\hbar^{2}}{2 m}\left[\left(k-\frac{2 \pi}{a} l\right)^{2}-k^{2}\right] \\
& =\frac{2 \hbar^{2} \pi}{m a} l\left(\frac{\pi l}{a}-k\right)
\end{aligned}
$$

ou seja, para $k=\pi l / a$, o denominador de 3.19 se anula. Isso ocorre devido à dege-

\footnotetext{
${ }^{1}$ Usando teoria de perturbação para sistemas não degenerados

${ }^{2}$ Como $V(x)$ é periódica, podemos expandi-la em uma série de Fourier, e como a e exponencial pode ser escrita como $e^{i\left(k-k^{\prime}\right) x}=\cos \left(k-k^{\prime}\right) x+i \operatorname{sen}\left(k-k^{\prime}\right) x$, temos que a integral se anula termo a termo devido à ortogonalidade dos senos e cossenos.

${ }^{3}$ Novamente, usando teoria de perturbação para sistemas não degenerados
} 
nerescência que surge nas vizinhanças da superfície de Fermi e portanto neste caso é necessária a utilização da teoria de perturbação para estados degenerados. Na teoria de perturbação para estados degenerados, deve-se diagonalizar o hamiltoniano total no subespaço dos estados degenerados $\psi_{k}^{(0)}(x)$ e $\psi_{k^{\prime}}^{(0)}(x)[31]$, com $k=\pi l / a$ e $k^{\prime}=-\pi l / a$. A matriz que representa $H$ nesta base é

$$
H \doteq\left(\begin{array}{ll}
E_{k}^{(0)} & V_{k k^{\prime}} \\
V_{k^{\prime} k} & E_{k^{\prime}}^{(0)}
\end{array}\right)
$$

com $V_{k^{\prime} k}=V_{k k^{\prime}}^{*}$ devido ao fato de $V(x)$ ser hermitiano. Os autovalores deste operador neste subespaço são as correções de primeira ordem na energia,

$$
E_{ \pm, k k^{\prime}}=\frac{1}{2}\left(E_{k}^{(0)}+E_{k^{\prime}}^{(0)}\right) \pm \sqrt{\frac{1}{4}\left(E_{k}^{(0)}-E_{k^{\prime}}^{(0)}\right)^{2}+\left|V_{k k^{\prime}}\right|^{2}}
$$

para $\left|E_{k}^{(0)}-E_{k^{\prime}}^{(0)}\right| \gg\left|V_{k k^{\prime}}\right|$, recuperamos as energias não perturbadas $E_{k}^{(0)}$ e $E_{k^{\prime}}^{(0)}$, mas para valores de $k$ próximos à superfície de Fermi, temos

$$
\Delta E=E_{+, k \rightarrow l \pi / a}-E_{-, k \rightarrow l \pi / a}=2\left|V_{k k^{\prime}}\right|
$$

Com isto podemos notar que há a abertura de um gap nas regiões próximas à superfície de Fermi, como ilustrado na figura 3.4.

Para um sistema com a banda de valência preenchida em $1 / n$ de sua totalidade, a cadeia unidimensional sofre então uma distorção de modo que sua célula unitária se torna $n$ vezes maior e sua nova banda de valência, que se estende entre $-\pi / n a$ e $\pi /$ na fica totalmente preenchida. Deste modo, ocorre o surgimento de um gap entre as bandas de valência e condução como ilustrado na figura 3.4, fazendo com que sistemas unidimensionais em que se esperava um comportamento condutor sejam de fato semicondutores. [32] 


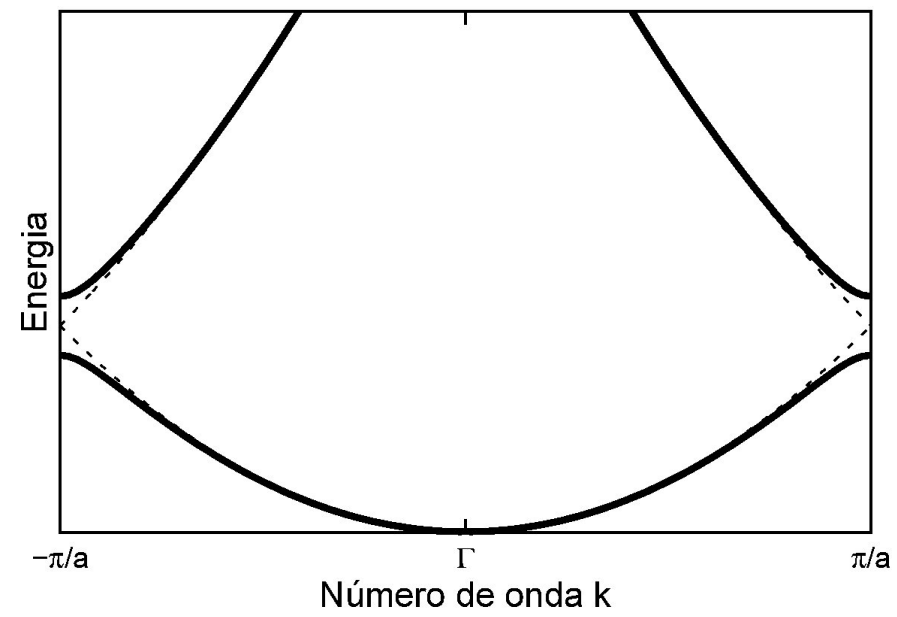

Figura 3.4.: Gráfico da dispersão para o sistema unidimensional. As linhas tracejadas são obtidas com o sistema não perturbado e as linhas cheias com a perturbação[19]. 



\section{Metodologia}

Neste trabalho foram utilizados os pacotes de programas Gaussian 03 [33], Gaussian 09 [34] e Cerius $^{2}$ [35]. Com os dois primeiros, a parte do trabalho baseada em Mecânica Quântica e Teoria do Funcional da Densidade - DFT - (apêndice B) foi feito. Com o último, foram realizados cálculos baseados em Mecânica Clássica para minimização da energia potencial através da utilização de um campo de forças clássico (apêndice D) e para a obtenção das trajetórias dos sistemas, através da integração das equações de movimento de modo a investigar a interação entre os nanotubos e o pentaceno.

\subsection{Mecânica Quântica - DFT}

Nesta parte, as equações de Kohn-Sham foram resolvidas numericamente, fornecendo a energia, orbitais de Kohn-Sham, densidade eletrônica, estrutura de bandas e configuração de equilíbrio do estado fundamental dos sistemas de interesse. Os funcionais de troca e correlação escolhidos para os cálculos foram o B3LYP e PBE. Estes funcionais foram escolhidos por se encontrarem entre os funcionais mais mais modernos e que apresentam os resultados mais condizentes com dados experimentais [36, 37]. O PBE [38] é um funcional do tipo GGA (Generalized Gradient Approach), ou seja, apresenta uma dependência em relação ao gradiente da densidade eletrônica em cada ponto do espaço na sua forma funcional. O B3LYP [39] é um funcional híbrido, que além de ter dependência da densidade e das derivadas da densidade eletrônica, (ou seja, também é do tipo GGA) apresenta uma contribuição do termo de troca exato de 
Hartree-Fock, o que torna este funcional capaz de descrever de maneira mais próxima da realidade sistemas orgânicos conjugados [37, 40]. As bases escolhidas para a obtenção dos orbitais de Kohn-Sham e consequentemente a densidade eletrônica foram bases gaussianas do tipo split-valence, nas quais os orbitais atômicos são representados como combinações lineares de funções gaussianas contraídas [41]. Mesmo para os sistemas com periodicidade, tais bases foram escolhidas em detrimento das bases de ondas planas, muito utilizadas para tal tipo de cálculo [41, 42]. Isso deve-se ao fato de que sistemas com periodicidade em uma única direção - como é o caso dos nanotubos ou polímeros - apresentam grandes variações de densidade eletrônica ao longo das direções perpendiculares ao seu eixo. Com isso, é necessária a utilização da combinação de um número muito grande de funções do tipo ondas planas para descrever os orbitais cristalinos destes sistemas, o que torna o cálculo complexo computacionalmente. Em todos cálculos desta etapa, grade de integração foi de 99 pontos na direção radial e 590 pontos na direção angular para cada ponto na direção radial, descrevendo uma superfície esférica [43]).

Como um teste da metodologia, as energias, as conformações de equilíbrio, as bandas e a Densidade de Estados (DOS ${ }^{1}$, de agora em diante) do poliacetileno nas formas cis-transóide, trans-cisóide e trans (figura 2.2) foram obtidas no estado fundamental, utilizando-se os funcionais PBE e B3LYP (utilizando-se $20 \%$ do termo de troca de Hartree-Fock) com a base gaussiana 6-311g(d). O cálculo foi realizado com condições periódicas de contorno na direção da cadeia do polímero, utilizando-se 200 pontos $k$. Com isto, foi possível obter o gráfico da estrutura de bandas e a DOS foi obtida através do cálculo de um histograma da estrutura de bandas com 1000 canais de igual largura compreendidos entre os pontos $k$ de maior e menor energia de todas as bandas.

Um estudo do efeito do termo de troca exato (equação B.15) na estrutura da ca-

${ }^{1}$ Density Of States 
deia, energia total, gap e comprimento do vetor de traslação da cadeia foi realizado, obtendo-se estas grandezas com funcional B3LYP com valores entre 0\% e $20 \%$ para a contribuição deste termo. Para cada valor da porcentagem do termo de troca utilizada, a estrutura dos polímeros foi otimizada e as outras grandezas foram obtidas para esta estrutura otimizada.

Os mesmos funcionais foram utilizados para a obtenção da conformação de menor energia, orbitais de Kohn-Sham, densidade eletrônica e energia total do estado fundamental dos nanotubos $(5,5)$ e $(9,0)$ com a base gaussiana $6-31 \mathrm{~g}(\mathrm{~d})$. Para estes cálculos, foram utilizadas condições períodicas de contorno ao longo do eixo do tubo com células unitárias unidimensionais que eram o triplo da célula unitária mínima. Utilizando uma rede de 400 pontos $k$, foram obtidas desde a décima banda abaixo da banda de valência em energia $\left(\mathrm{HOCO}^{2}-10\right)$ até a décima banda acima da banda de condução $\left(\mathrm{LUCO}^{3}+10\right)$. Da mesma forma que para o poliacetileno, a Densidade de Estados para estes dois funcionais foi obtida através da confecção de um histograma da estrutura de bandas com 1000 canais e o efeito do termo de troca exato para a estrutura dos tubos, energia total, gap, comprimento do vetor de translação da rede e raio dos tubos foi estudado da mesma maneira como foi descrito anteriormente para os polímeros.

A obtenção destas grandezas para os nanotubos foi particularmente complicada e demorada, pois os softwares Gaussian 03 e Gaussian 09 apresentaram grandes problemas de convergência para a estrutura eletrônica. Ao introduzir o termo de troca HF no DFT, problemas como o aparecimento de cargas na célula unitária começaram a ocorrer, tornando a convergência impossível, uma vez que a energia do sistema nestes casos atingia valores absurdamente grandes. Isso ocorreu devido a alguns fatores, em

\footnotetext{
${ }^{2}$ Highest Occupied Crystal Orbital - Mais alto orbital cristalino ocupado

${ }^{3}$ Lowest Unoccupied Crystal Orbital - Mais baixo orbital cristalino desocupado
} 
particular pelo fato que o elemento de matriz do termo de troca HF apresentava um decaimento em função da distância mais demorado que os outros elementos de matriz do funcional. Tal decaimento tornava-se ainda mais demorado com a utilização de funções difusas, [44] que de fato foram utilizadas nos átomos de carbono para melhor descrição da estrutura eletrônica e de bandas.

No caso do software utilizado, o cálculo de elementos de matriz problemáticos como este é feito separando-se o campo coulombiano em campo próximo e campo distante. Para o campo próximo, a expressão analítica é calculada numericamente e para campo distante é utilizada uma expansão em multipolos [45, 46]. O ajuste do parâmetro de separação do campo era também um processo determinante na convergência do cálculo. O grau desta expansão em multipolos também teve que ser finamente ajustado para que a integração fosse precisa o suficiente de modo que a carga obtida através da integração da densidade eletrônica fosse de fato igual em módulo à carga dos núcleos na célula unitária.

Outro fator determinante foi a escolha do número de pontos $k$ utilizados no cálculo, uma vez que alguns dos sistemas possuíam um gap muito pequeno, a ocupação em alguns ciclos dependia de $k$, o que levou à necessidade de utilização de um número grande de pontos $k$ (200 pontos para o poliacetileno e 400 para os nanotubos) para que a convergência fosse alcançada.

Para maiores detalhes, veja o apêndice C.

Para contornar essas dificuldades, foi necessário obter a configuração de menor energia para os nanotubos utilizando-se valores crescentes do peso do termo de troca HF, e a densidade eletrônica convergida em cada passo foi utilizada como a densidade inicial no passo seguinte, tomando-se todos os cuidados necessários ao ajustar o grau da expansão em multipolos, sua separação e o número de pontos $k$ utilizados em cada passo. 


\subsection{Mecânica Molecular}

Para a realização da Dinâmica Molecular, as configurações de equilíbrio dadas pela DFT para o pentaceno e os tubos foram inseridas no software Cerius $^{2}$. Com a coordenada da célula unitária de cada um dos tubos fornecida pelo Gaussian, foi possível construir uma célula unitária para os mesmos no Cerius $^{2}$, que no entanto não era periódica somente na direção do eixo do tubo, mas em todas as direções. Para que a interação entre os constituintes da célula unitária com seus vizinhos pudesse ser desprezada nas direções perpendiculares ao eixo do tubo, a célula foi construída com dimensões bem maiores nestas direções $(100 \AA)$ do que na direção do eixo do tubo $(\approx 35 \AA$ para o tubo $(5,5)$ e $\approx 25 \AA$ para o tubo $(9,0))$.

A estrutura do nanotubo isolado dentro desta célula foi relaxada, através da Mecânica Molecular, mantendo-se os parâmetros da célula fixos e utilizando-se o campo de forças CVFF 950[47]. O mesmo foi feito para o pentaceno isolado, no entanto sem utilizar tais condições periódicas de contorno. Com isto, as energias do nanotubo $\left(E_{n t}\right)$ e pentaceno $\left(E_{\text {pent }}\right)$ isolados foram obtidas. Em seguida, o pentaceno foi inserido na célula e a geometria de menor energia deste sistema foi obtida, assim como a energia do mesmo, para o sistema neutro e carregado (pentaceno doando um elétron para o nanotubo). A energia de ligação do sistema nestes dois casos foi então calculada através de

$$
E_{\text {lig }}=E_{n t+p e n t}-E_{n t}-E_{\text {pent }}
$$

Logo após, o mesmo procedimento foi repetido para o sistema nanotubo e pentaceno, mas com um número crescente de moléculas de pentaceno ao redor do tubo em diferentes configurações, para o caso em que todas as moléculas de pentaceno e o nanotubo tinham carga líquida nula e para o caso em que uma das moléculas mais 
próximas à parede do tubo e o tubo possuíam carga líquida $-e \mathrm{e}+e$, respectivamente. Neste caso, a energia de ligação do sistema é dada por

$$
E_{\text {lig }}=E_{n t+N p e n t}-E_{n t}-N E_{\text {pent }}
$$

onde $N$ é o número de moléculas de pentaceno posicionadas ao redor da parede externa do tubo.

Todas as configurações foram salvas após a Mecânica Molecular, e em posse destas configurações, as trajetórias dos átomos constituintes do sistema foram obtidas através de Dinâmica no ensemble NVT - número de partículas, volume e temperaturas constantes - por 1 ns com passos de integração de $1 \mathrm{fs}$ e termostato Nosé-Hoover. Com isto, foi possível investigar se de fato haveria a formação de aglomerados ou cristais de pentaceno próximos à parede externa dos nanotubos, e como seria a disposição de tais estruturas. 


\section{Resultados - DFT}

O estudo preliminar da metodologia, com os resultados da DFT para o poliacetileno é apresentado na primeira seção. Em seguida, os resultados da DFT obtidos para o pentaceno e nanotubo separadamente são apresentados.

\subsection{Poliacetileno}

A estrutura do cis-poliacetileno foi calculada para duas configurações iniciais: a trans-cisóide e a cis-transóide. A configuração cis-transóide apresentou menor energia total com o funcional B3LYP (com 20\% do termo de troca de HF) enquanto que a trans-cisóide apresentou menor energia total com o funcional PBE, mas pelo fato desta diferença de energia nos dois casos ser menor que $1 \mathrm{meV}$, as geometrias resultantes de ambas as configurações foram consideradas equivalentes e denominadas simplesmente de $c i s$-poliacetileno.

Os comprimentos das ligações entre os átomos de carbono obtidos para os funcionais

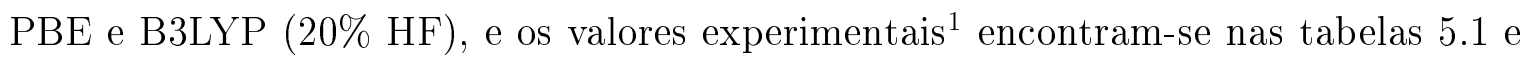
5.2 .

Pode-se notar que a alternância entre as ligações $C-C$ é mais acentuada nos resultados obtidos com o B3LYP, que também encontram-se mais próximos dos resultados experimentais [48].

\footnotetext{
${ }^{1}$ Os valores experimentais na tabela 5.1 são apenas para a configuração cis-transóide, pois a conformação trans-cisóide não foi observada experimentalmente
} 


\begin{tabular}{lcc}
\multicolumn{3}{c}{ cis-poliacetileno } \\
\hline Funcional & A & B \\
\hline B3LYP (20\% HF) & 1,373 & 1,437 \\
PBE & 1,391 & 1,432 \\
\hline Experimental & $1,37 \pm 0,01$ & -
\end{tabular}

Tabela 5.1.: Comprimentos de ligação $(\AA)$ entre os átomos de carbono na estrutura do cis-poliacetileno obtidos com os funcionais B3LYP $(20 \% \mathrm{HF})$ e PBE, e os valores experimentais [48]. O código das ligações encontra-se na figura $5.5(\mathrm{a})$.

\begin{tabular}{lcc}
\multicolumn{3}{c}{ trans-poliacetileno } \\
\hline Funcional & A & B \\
\hline B3LYP (20\% HF) & 1,373 & 1,426 \\
PBE & 1,399 & 1,415 \\
\hline Experimental & $1,36 \pm 0,01$ & $1,44 \pm 0,01$
\end{tabular}

Tabela 5.2.: Comprimentos de ligação $(\AA)$ entre os átomos de carbono na estrutura do trans-poliacetileno obtidos com os funcionais B3LYP (20\% HF) e PBE, e os valores experimentais [48]. O código das ligações encontra-se na figura 5.5 (b).

Como as duas configurações do poliacetileno na forma cis apresentaram estruturas de bandas praticamente iguais para os dois funcionais utilizados, apresentamos as bandas e $\operatorname{DOS}^{2}$ da geometria cis-transóide apenas, pois as diferenças são imperceptíveis. Os resultados encontram-se nas figuras 5.1 e 5.2 a seguir.

É notável a abertura de um gap ao redor da energia de Fermi para os cálculos com o funcional B3LYP para ambas as formas do poliacetileno $(\approx 1,20 \mathrm{eV}$ para a forma trans $\mathrm{e} \approx 1,9 \mathrm{eV}$ para a forma cis) e para o cálculo com o funcional PBE para a forma cis $(\approx 0,8 \mathrm{eV})$. Comparando os dois resultados para a forma cis, pode-se notar que o gap é maior para o B3LYP, que também está mais próximo do valor experimental $(\approx$ $1,8 \mathrm{eV})$ [48]. Para a forma trans, o PBE também fornece um gap muito pequeno $(0,16$ eV) mas pelo fato do mesmo ser muito pequeno, podemos dizer que os resultados do PBE não estão de acordo com o valor experimental.

${ }^{2}$ Density Of States - Densidade de Estados 


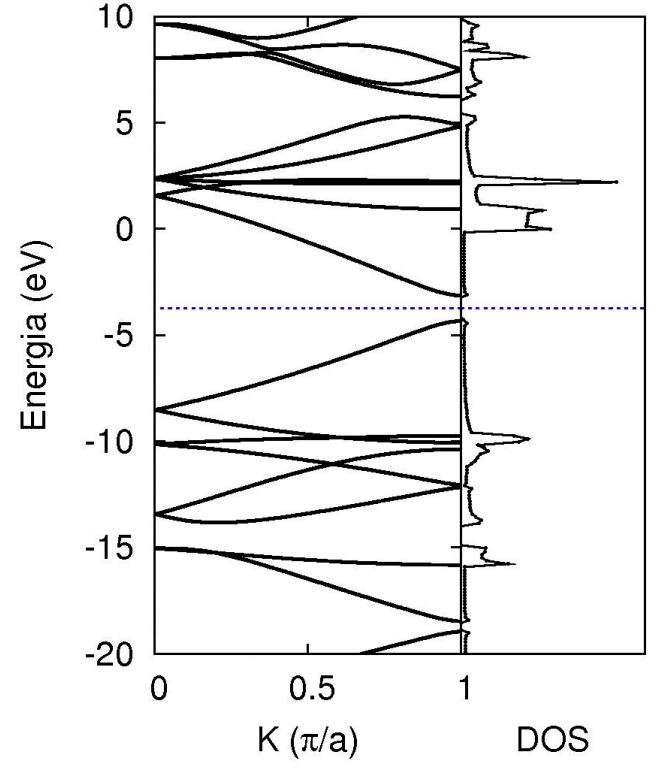

(a)

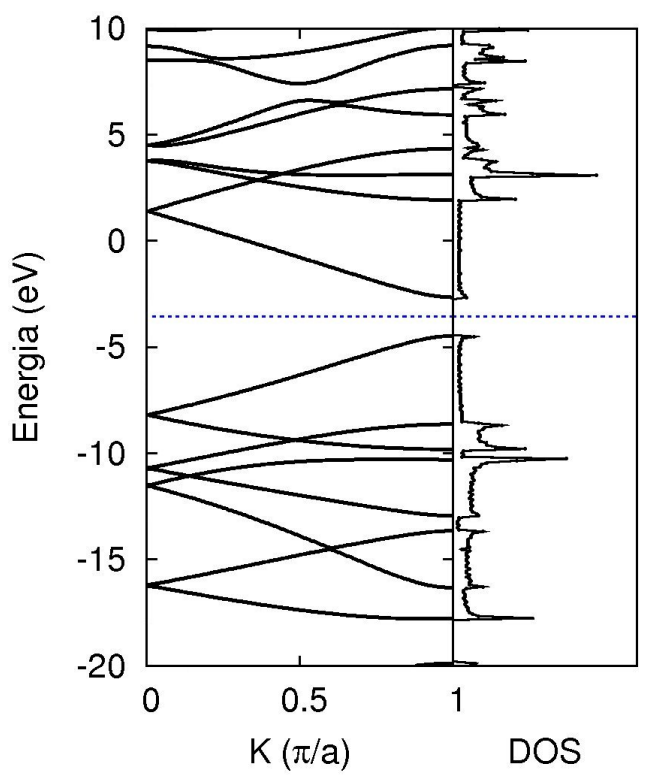

(b)

Figura 5.1.: Gráficos das bandas e da densidade de estados para o poliacetileno na forma trans (a) e cis (b) calculados com o funcional B3LYP (20\% HF). A linha tracejada horizontal é a energia de Fermi.

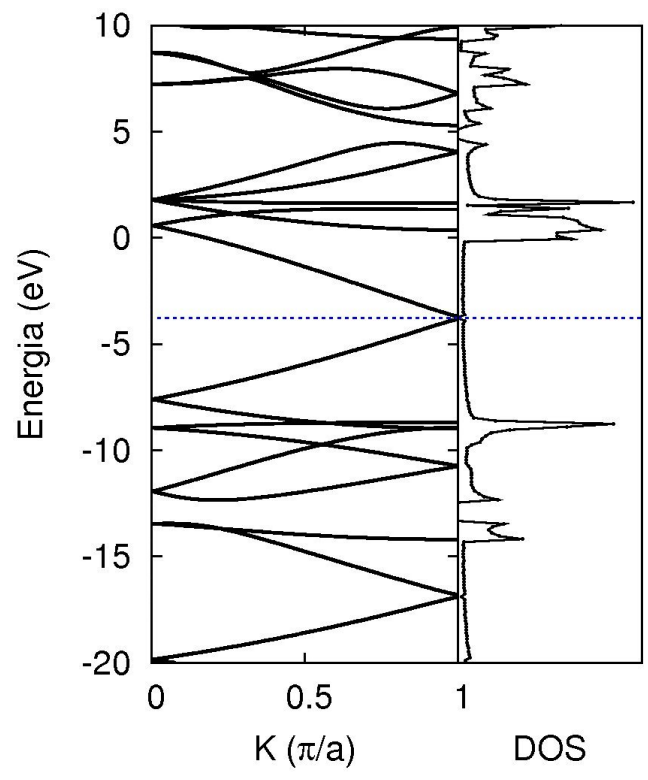

(a)

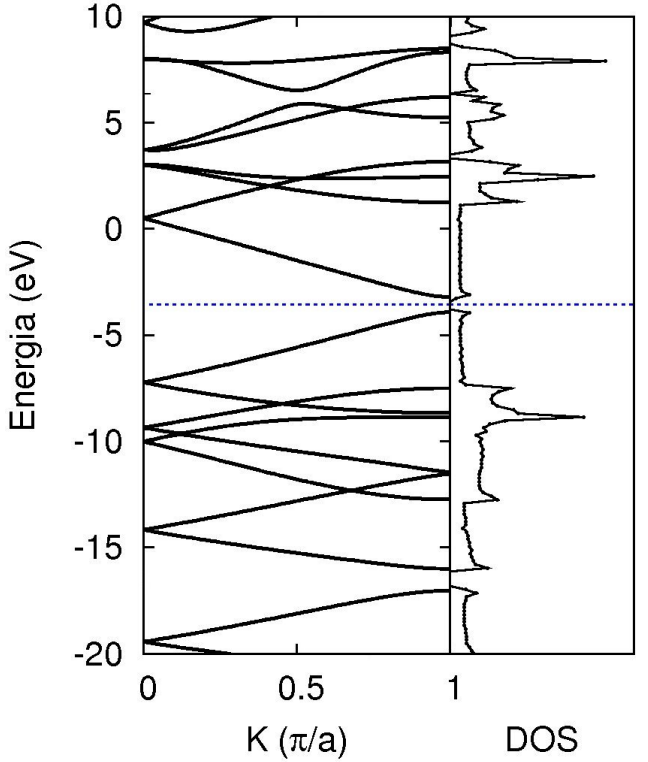

(b)

Figura 5.2.: Gráficos das bandas e da densidade de estados para o poliacetileno na forma trans (a) e cis (b) calculados com o funcional PBE. A linha tracejada horizontal é a energia de Fermi. 
Para investigar o efeito do termo de troca exato introduzido no funcional B3LYP, as estruturas de equilíbrio do poliacetileno nas forma trans e cis (figura 2.2) foram obtidas utilizando-se DFT com os funcionais B3LYP e PBE, com a base gaussiana 6-311g(d). Fizemos variar desde $0 \%$ até $20 \%$ a contribuição do termo de troca de Hartree-Fock (HF) no B3LYP (equações B.15 e B.16).

Foi observado que com isto, a energia total do sistema diminuia linearmente com o aumento desta contribuição, como pode ser observado nos gráficos das figuras 5.3. Isso pode ser explicado pelo fato que o termo de troca de Hartree-Fock diminui a repulsão entre elétrons de mesmo spin em orbitais distintos. Com isto, pode-se explicar também o fato de que o comprimento do vetor de translação da célula unitária diminuia com o aumento do termo de HF, conforme pode ser notado nos gráficos das figuras 5.4.

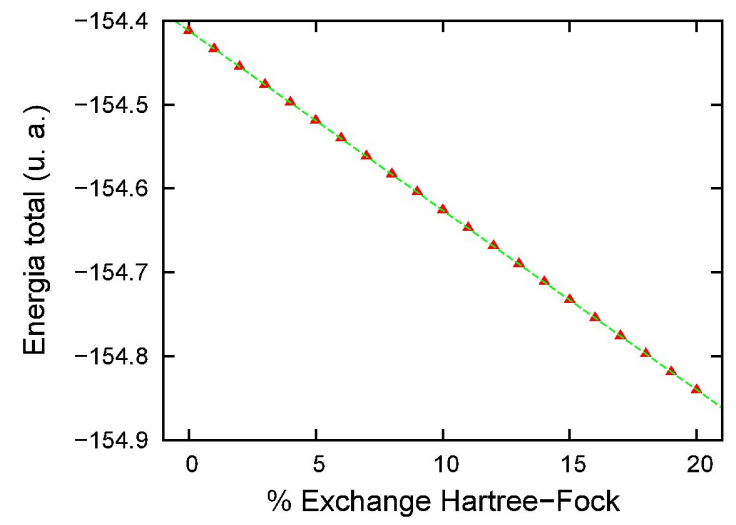

(a)

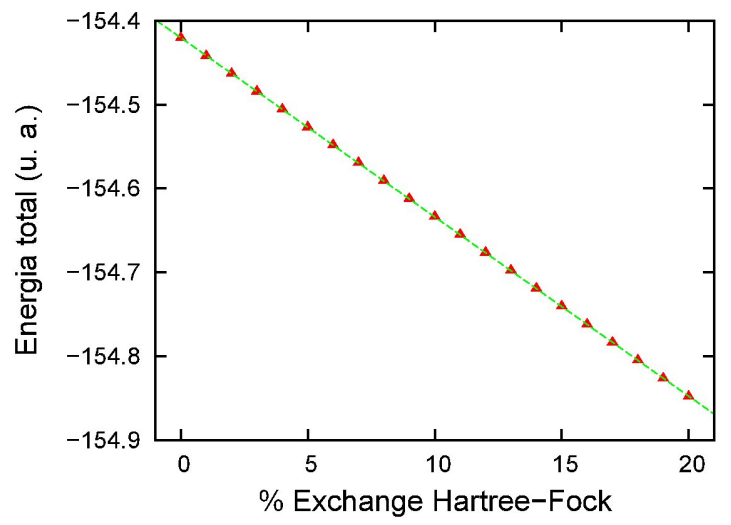

(b)

Figura 5.3.: Gráficos da energia total em função da porcentagem de exchange de Hartree-Fock para o (a) cis-poliacetileno e (b) trans-poliacetileno

Finalmente, os comprimentos de ligação em função do termo de HF evidenciaram o aumento da alternância entre as ligações à medida que adicionamos uma contribuição maior do termo de troca (figura 5.5) assim como o gap (figura 5.6). Para os gráficos do trans-poliacetileno, é possível notar que os pontos não descrevem uma curva tão 


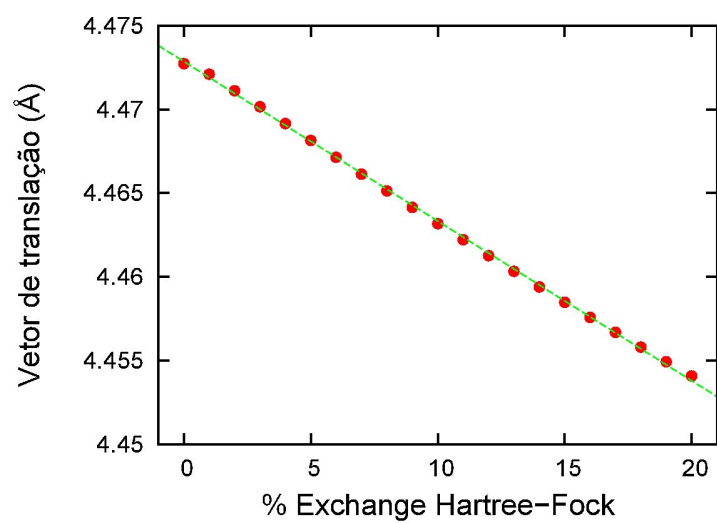

(a)

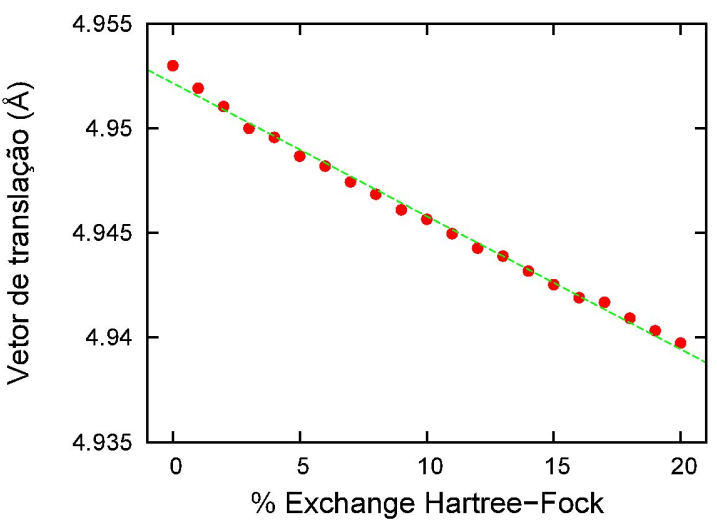

(b)

Figura 5.4.: Gráficos do comprimento do vetor de translação da rede direta em função da porcentagem de exchange de Hartree-Fock para o (a) cis-poliacetileno e (b) trans-poliacetileno

suave quanto para a forma cis, tanto no gráfico dos comprimentos de ligação quanto no gráfico do gap. Isso sugere que ambas as grandezas - a alternância de ligações e o gap - apresentam alguma relação, sugerindo a existência da distorção de Peierls neste sistema.

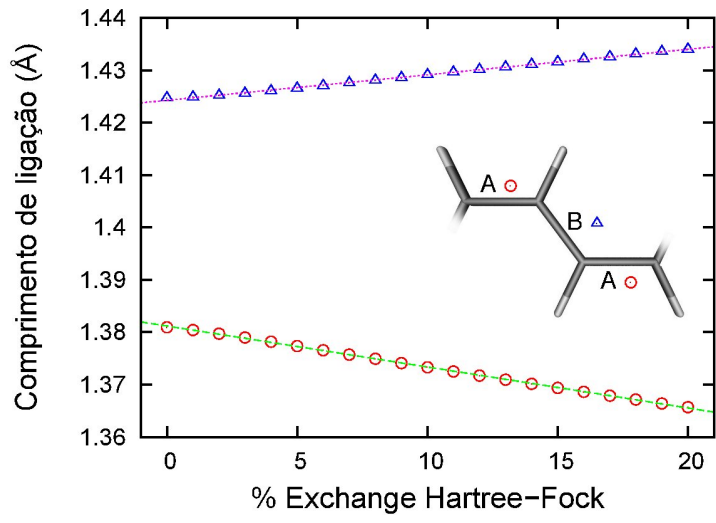

(a)

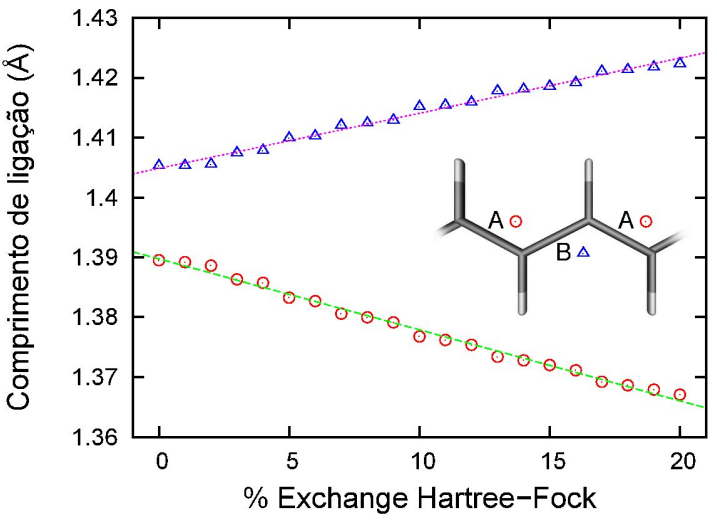

(b)

Figura 5.5.: Gráficos dos comprimentos de ligação em função da porcentagem de exchange de Hartree-Fock para o (a) cis-poliacetileno e (b) transpoliacetileno

Como o funcional B3LYP apresentou valores cada vez mais próximos ao valor ex- 


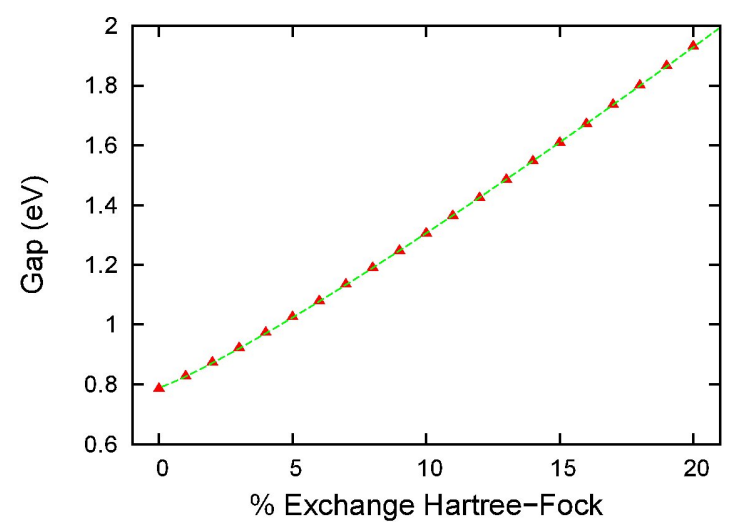

(a)

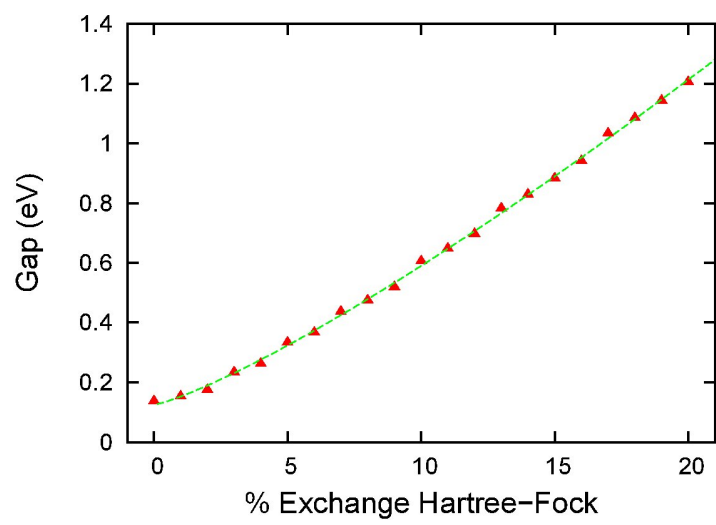

(b)

Figura 5.6.: Gráficos do gap em função da porcentagem de exchange de Hartree-Fock para o (a) cis-poliacetileno e (b) trans-poliacetileno

perimental para o gap à medida que o termo de exchange de HF foi sendo adicionado, alcançando os valores de 1,9 eV e 1,2 eV para as formas cis e trans respectivamente para $20 \%$ de exchange de $\mathrm{HF}$, como pode ser notado pelo comportamento do gráfico da figura 5.6, são esperado valores ainda mais próximos do valor experimental para contribuições maiores do termo de exchange HF.

Tais resultados evidenciam que o funcional B3LYP é mais adequado para o tratamento deste sistema do que o funcional PBE, que é um dos mais avançados funcionais do tipo GGA.

\subsection{Pentaceno}

Os resultados para a geometria do pentaceno, que foram obtidos utilizando-se os funcionais B3LYP e PBE com a base gaussiana 6-31g, e os comprimentos das ligações obtidas, assim como os valores experimentais [49], encontram-se na tabela 5.3.

Pode-se notar que o PBE forneceu todos os comprimentos de ligação maiores que o B3LYP, que por sua vez ficaram mais próximos dos valores experimentais, indicando que o funcional B3LYP é o mais apropriado para estudar a estrutura de moléculas 
deste tipo.

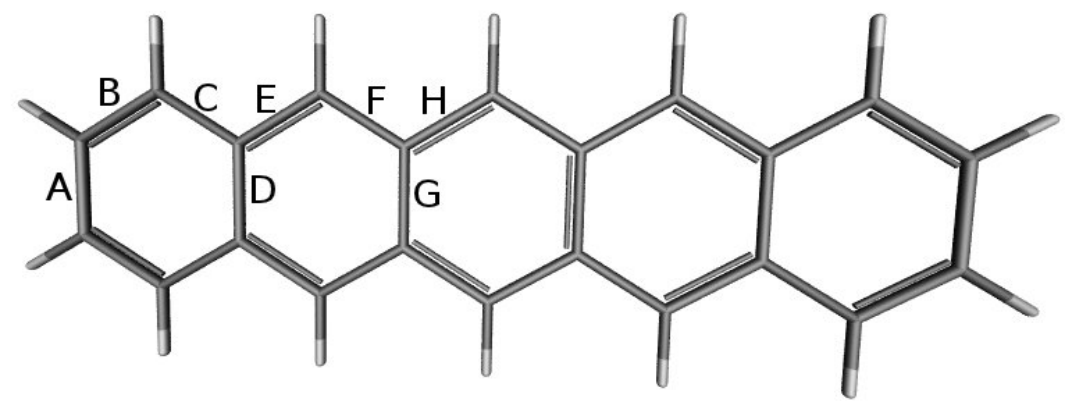

Figura 5.7.: Ligações entre os átomos de Carbono da molécula de pentaceno. Devido à simetria da molécula, (grupo pontual $D_{2} H$ ) as ligações são as mesmas para as partes não rotuladas.

\begin{tabular}{lcccccccc} 
Funcional & A & B & C & D & E & F & G & H \\
\hline B3LYP (20 \% HF) & 1,435 & 1,370 & 1,439 & 1,459 & 1,392 & 1,418 & 1,461 & 1,405 \\
PBE & 1,437 & 1,380 & 1,440 & 1,465 & 1,402 & 1,421 & 1,468 & 1,412 \\
Experimental & 1,43 & 1,35 & 1,42 & 1,44 & 1,38 & 1,40 & 1,45 & 1,39 \\
\hline
\end{tabular}

Tabela 5.3.: Comprimentos da ligações para o pentaceno obtidos teoricamente e experimentalmente [49], em Å. O código das ligações está de acordo com a figura 5.7

\subsection{Nanotubos}

Os comprimentos das ligações ao longo do eixo dos nanotubos $(5,5)$ e $(9,0)$, obtidos com o funcional B3LYP com 20\% de exchange Hartree-Fock e com o funcional PBE, ambos utilizando-se uma célula unitária três vezes maior do que a célula unitária mínima, encontram-se nas tabelas 5.4 e 5.5 respectivamente, com o código de letras dado pela figura 5.8. 


\begin{tabular}{|c|c|c|c|}
\hline \multicolumn{4}{|c|}{ B3LYP - $20 \%$ HF } \\
\hline Ligação & $\mathrm{A}$ & $\mathrm{B}$ & $\mathrm{C}$ \\
\hline Comprimento & 1,427 & 1,428 & 1,430 \\
\hline \multicolumn{4}{|c|}{ PBE } \\
\hline Ligação & $\bar{A}$ & $\mathrm{~B}$ & $\mathrm{C}$ \\
\hline Comprimento & 1,433 & 1,433 & 1,435 \\
\hline
\end{tabular}

Tabela 5.4.: Comprimentos das ligações do tubo $(5,5)$ (figura 5.8 - a), em A, para os funcionais B3LYP (20\% HF) e PBE

\begin{tabular}{lcc}
\multicolumn{4}{c}{ B3LYP -20} & \% HF \\
\hline Ligação & $\mathrm{A}$ & $\mathrm{B}$ \\
Comprimento & 1,433 & 1,418 \\
\hline \multicolumn{3}{c}{ PBE } \\
\hline Ligação & $\mathrm{A}$ & $\mathrm{B}$ \\
Comprimento & 1,438 & 1,424 \\
\hline
\end{tabular}

Tabela 5.5.: Comprimentos das ligações do tubo (9,0) (figura 5.8 - b), em $\AA$, para os funcionais B3LYP (20\% HF) e PBE

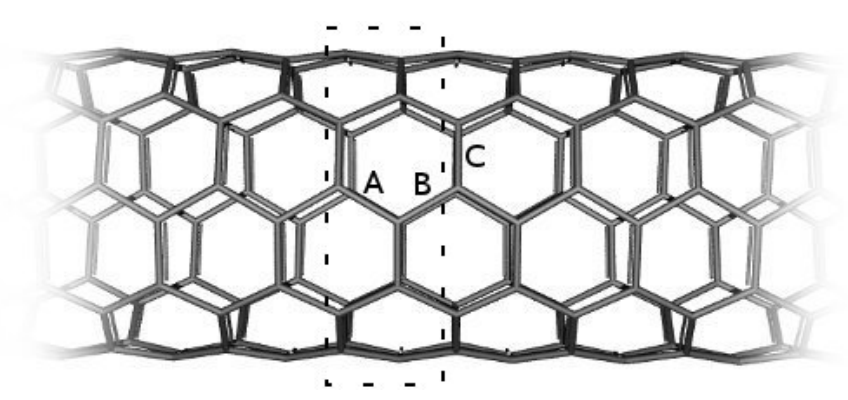

(a)

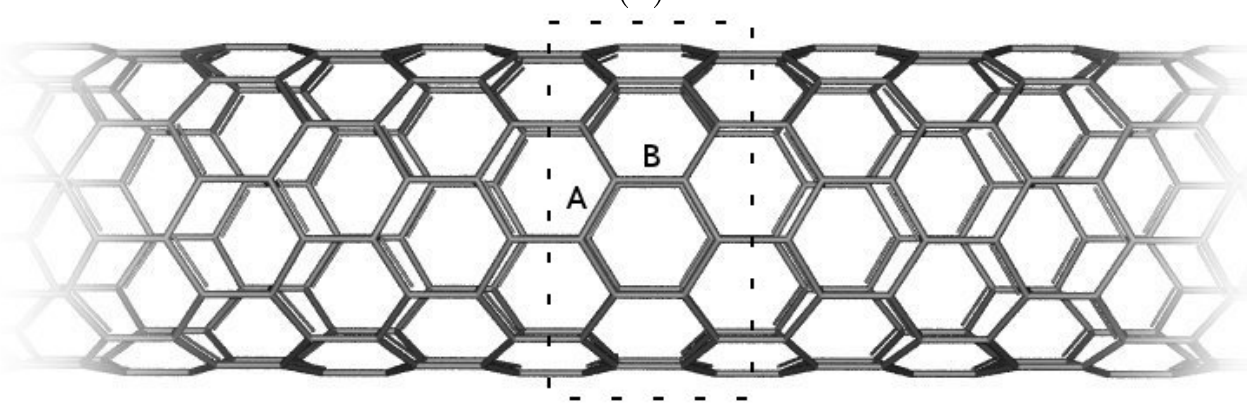

(b)

Figura 5.8.: Estrutura dos nanotubos $(5,5)$ (a) e $(9,0)$ (b). A área tracejada indica uma célula unitária. 
Medidas precisas da estrutura dos nanotubos de carbono, como a determinação dos comprimentos de suas ligações $C-C$, não foram obtidas experimentalmente até o momento. De todos os métodos experimentais utilizados até a presente data para estudar nanotubos, a espectroscopia Raman é a mais conhecida. Com esta técnica, é possível obter a posição da banda do Modo de Respiro Radial (RBM - Radial Breathing Mode) e com isso é possível determinar o diâmetro dos nanotubos [50-52]. A presença de defeitos pode ser estimada observando-se a banda D [52] através da mesma técnica de espectroscopia. A determinação dos índices $(n, m)$ pode ser feita utilizando-se resultados de absorção óptica e Raman [51].

Mesmo com esta dificuldade, Kuzmany et al. indicam que a estrutura dos nanotubos pode apresentar desvios em relação à estrutura do grafeno, especialmente para nanotubos de diâmetro pequeno. Tais desvios estariam relacionados com a quiralidade dos tubos e com efeitos de curvatura da estrutura, segundo os autores [53].

Do mesmo modo que observado para o poliacetileno, os comprimentos das ligações são menores para o B3LYP, que no entanto forneceu uma maior alternância. Como os tubos estudados neste trabalho são tubos de diâmetro pequeno $(\approx 7 \AA$, de acordo com a equação 2.5), podemos interpretar esta alternância de ligações como uma possível explicação ao desvio da estrutura do grafeno mencionado anteriormente.

Os gráficos das bandas e da $\operatorname{DOS}^{3}$ para os nanotubos $(5,5)$ e $(9,0)$ obtidos com o funcional B3LYP (20 \% HF) e com o funcional PBE encontram-se nas figuras 5.9 e 5.10 respectivamente.

O tubo $(5,5)$ apresentou um gap de $0,13 \mathrm{eV}$ com o B3LYP e nulo para o PBE e o tubo $(9,0)$ apresentou um gap de 0,37 eV com o B3LYP e 0,10 eV com o PBE, como pode

\footnotetext{
${ }^{3}$ Density Of States - Densidade de Estados
} 


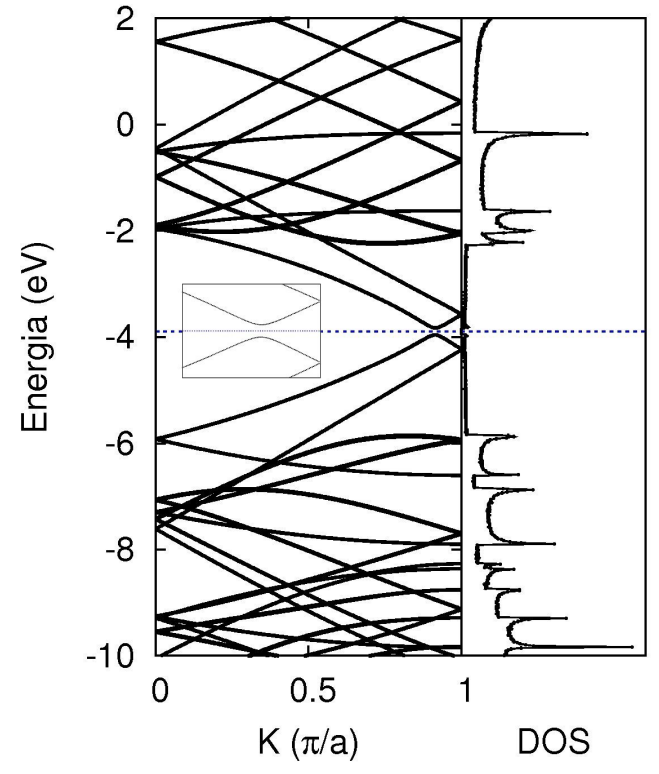

(a)

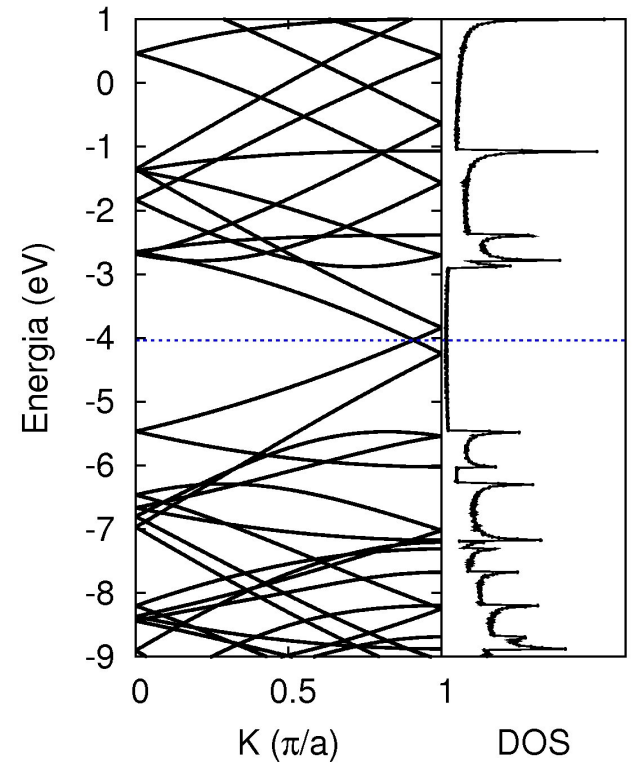

(b)

Figura 5.9.: Gráficos da estrutura de bandas e DOS para o tubo $(5,5)$ calculadas utilizando-se os funcionais B3LYP (20\% HF) (a) e PBE (b). A linha tracejada indica a energia de Fermi. Detalhe em (a) mostra o gap.

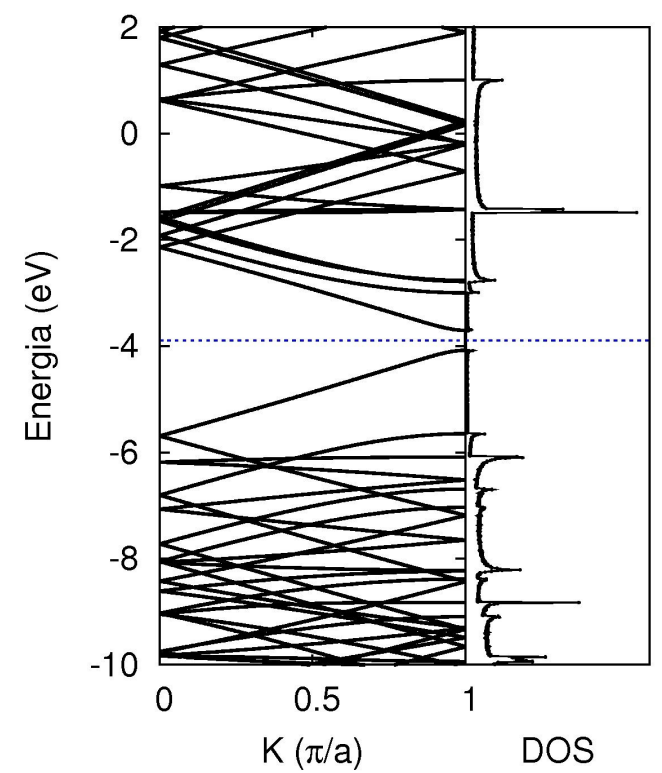

(a)

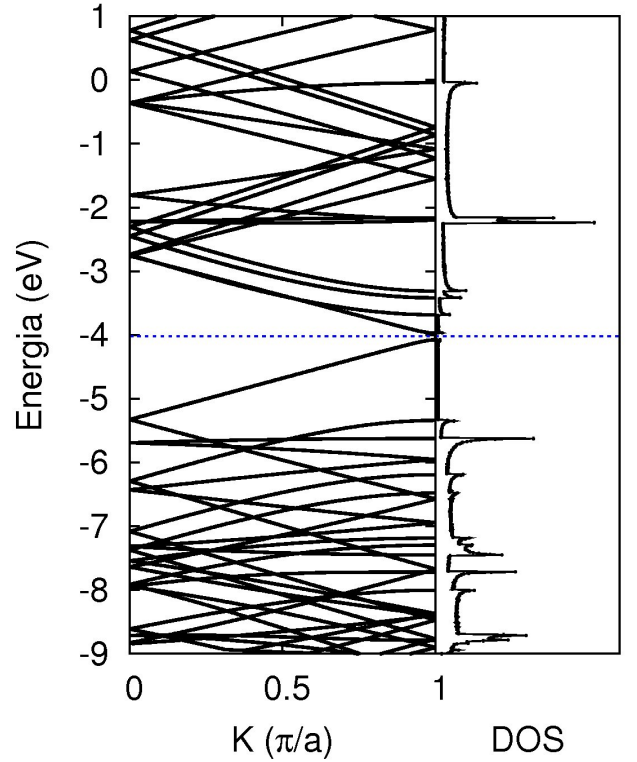

(b)

Figura 5.10.: Gráficos da estrutura de bandas e DOS para o tubo $(9,0)$ calculadas utilizando-se os funcionais B3LYP (20\% HF) (a) e PBE (b). A linha tracejada indica a energia de Fermi. 
ser observado nos gráficos. De fato, alguns autores já previram e até mesmo afirmam ter observado um pequeno gap para nanotubos ditos metálicos [11-13, 15, 17].

Entre estes autores, Reich et al. [12] utilizou DFT na aproximação LDA ${ }^{4}$ e obteve um gap de $20 \mathrm{meV}$ para o nanotubo $(9,3)$ isolado e $51 \mathrm{meV}$ para o nanotubo $(6,6)$ em um feixe, afirmando que o aparecimento do gap para o primeiro estava relacionado com a curvatura da estrutura do tubo, enquanto que para o segundo, a quebra de simetria do sistema quando em presença de outros tubos em um feixe era a responsável pela mistura dos orbitais $\pi$ e $\pi^{*}$ e consequente aparecimento do gap. Resultados experimentais parecidos foram obtidos por Ishii et al. através de PES $^{5}$ e por Ouyang et al. [13] com um microscópio de $\mathrm{STM}^{6}$ em ultra alto vácuo a $5 \mathrm{~K}$ para um tubo $(9,0)$ isolado sobre um substrato de $\mathrm{Au}(111)$ (gap $\approx 80 \mathrm{meV}$ ) e para um tubo $(8,8)$ em um feixe também sobre a mesma superfície (gap $\approx 80$ - $100 \mathrm{meV}$ ). Tais resultados encontram-se um pouco abaixo dos resultados obtidos neste trabalho, mas no entanto devemos levar em consideração que além da temperatura utilizada nos cálculos ser sempre zero kelvin a temperatura de transição de Peierls também é muito pequena, sendo estimada da ordem de poucos kelvins [15] o que pode introduzir diferenças nos resultados simulados e medidos.

Yumura et al. [17] utilizou DFT com o funcional B3LYP para simular os tubos $(5,5)$ e $(9,0)$ com as pontas fechadas por fulerenos e com diversos comprimentos. Ele obteve um comportamento periódico para o gap do tubo $(5,5)$ em função do comprimento (ou número de células unitárias adicionadas à estrutura) de período 3, o que evidencia que a nossa metodologia utilizando uma célula unitária triplicada para este sistema é correta. Os valores obtidos para o gap também estão condizentes com o valor calculado neste trabalho, encontrando-se entre aproximadamente $1,2 \mathrm{eV}$ e $2,7 \mathrm{eV}$ para o tubo

\footnotetext{
${ }^{4}$ Local Density Approach - Aproximação de Densidade Local

${ }^{5}$ PhotoElectron Spectroscopy - Espectroscopia de Foto Elétrons

${ }^{6}$ Scanning Tunneling Microscope - Microscópio de Escaneamento por Tunelamento
} 
$(9,0)$ e $0,7 \mathrm{eV}$ e $2,7 \mathrm{eV}$ para o tubo $(5,5)$.

A interpretação de Reich et al. e Ouyang et al., de que o aparecimento do gap para nanotubos armchair ocorre devido à quebra de simetria dos nanotubos quando organizados em feixes, pode ser contestada frente ao resultado obtido para o tubo $(5,5)$, que forneceu um gap maior mesmo estando isolado. Para estudar o efeito da curvatura, tubos maiores deveriam ser simulados.

Da mesma maneira que foi feito para o poliacetileno, o efeito da adição do termo de troca de Hartree-Fock para o cálculo dos nanotubos $(5,5)$ e $(9,0)$ com o funcional B3LYP (equações B.15 e B.16) foi investigado.

Observando-se o comportamento linear e decrescente para a energia total dos tubos em função do termo de HF, (figura 5.11) é possível dizer que, do mesmo modo que observado para o poliacetileno, este termo diminui a repulsão entre elétrons de mesmo spin em orbitais diferentes, tornando o sistema mais estável. O mesmo pode ser dito para a magnitude do vetor de translação da célula unitária e para o raio dos tubos em função da mesma grandeza, como evidenciado nas figuras 5.12 e 5.13, respectivamente.

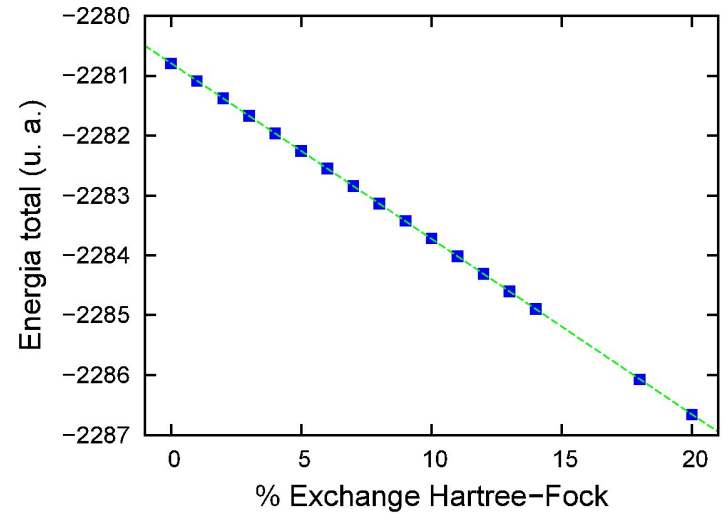

(a)

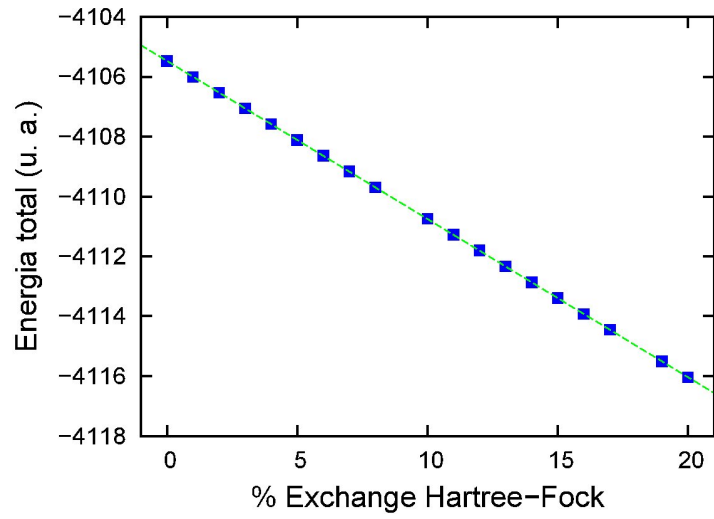

(b)

Figura 5.11.: Gráficos da energia total em função da porcentagem de exchange de Hartree-Fock para os nanotubos (a) $(5,5)$ e (b) $(9,0)$ 


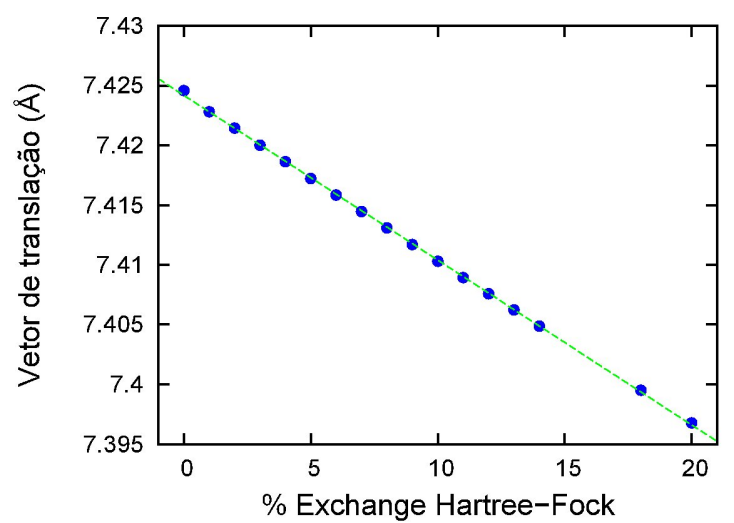

(a)

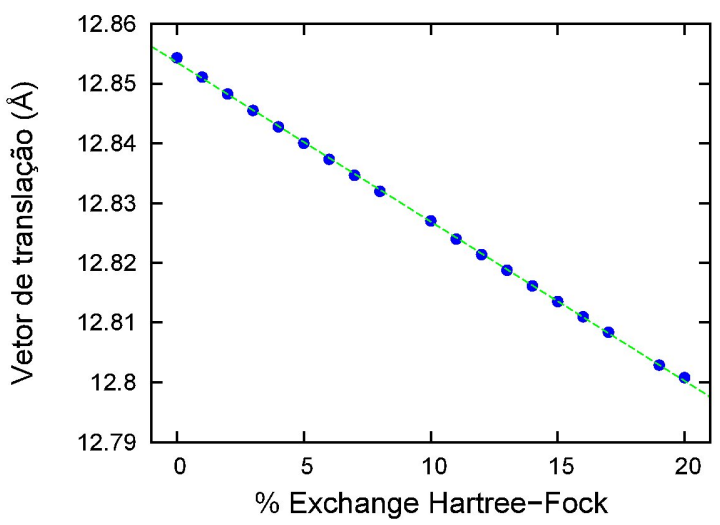

(b)

Figura 5.12.: Gráficos do comprimento do vetor de translação da rede direta em função da porcentagem de exchange de Hartree-Fock para os nanotubos (a) $(5,5)$ e (b) $(9,0)$

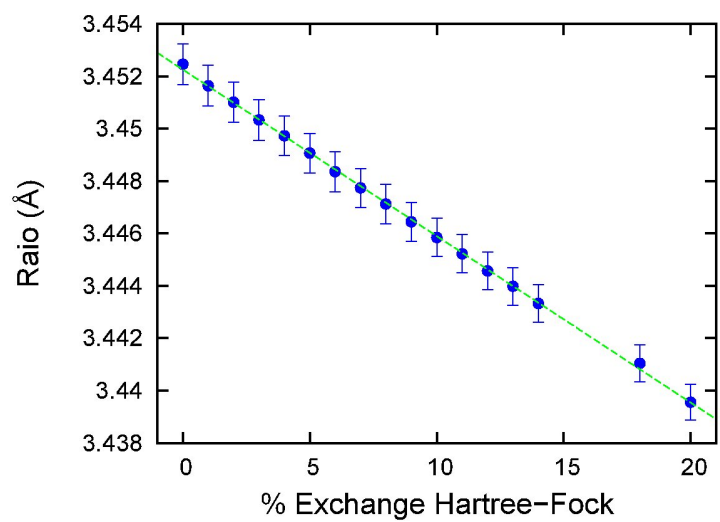

(a)

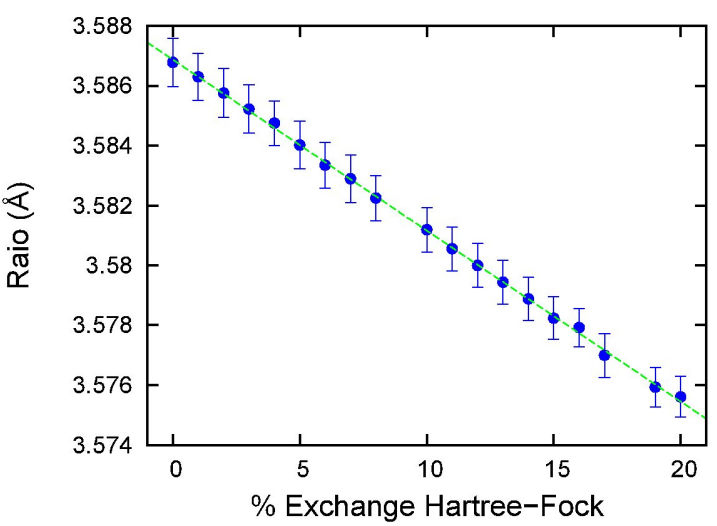

(b)

Figura 5.13.: Gráficos dos raios em função da porcentagem de exchange de HartreeFock para os nanotubos (a) $(5,5)$ e (b) $(9,0)$

Observando-se os gráficos dos comprimentos de ligação e do gap ${ }^{7}$ (figuras 5.14 e 5.15 respectivamente) em função do termo de HF, pode-se notar um fato que também pode ser interpretado como uma evidência da relação entre o gap e a distorção: para o tubo $(5,5)$, o gap torna-se não nulo apenas para valores de contribuição do termo HF a partir de 9\%, (conforme figura 5.15 - (a)) e é aproximadamente para estes valores de

\footnotetext{
${ }^{7}$ Os valores do gap do tubo $(5,5)$ para contribuições do termo de Hartree-Fock menores que $9 \%$ devem ser considerados nulos, pois se originam do fato que o software Gaussian sempre obtém este número através da diferença entre o ponto de maior energia da banda preenchida e o ponto de menor energia da banda vazia.
} 
contribuição HF que os comprimentos de ligação ao longo do eixo deste tubo começam a se tornar significativamente diferentes. (conforme figura 5.14 - (a))

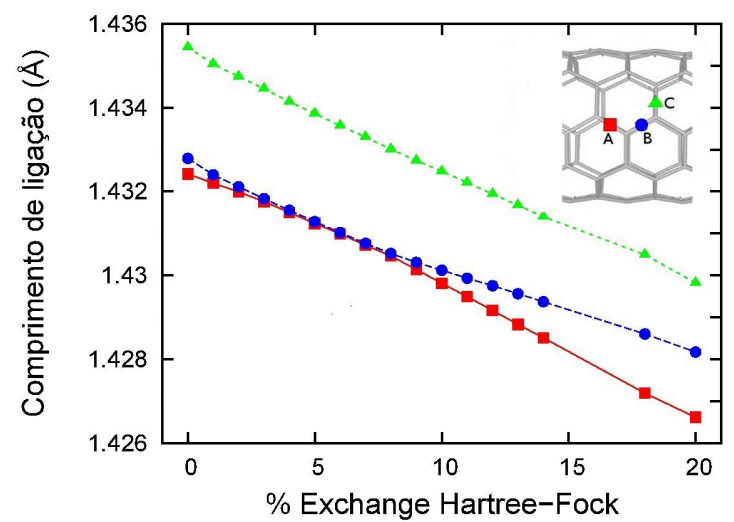

(a)

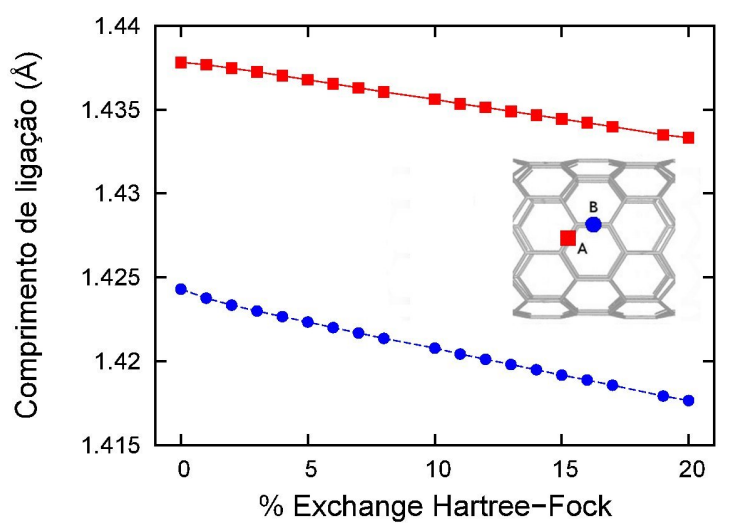

(b)

Figura 5.14.: Gráficos dos comprimentos de ligação em função da porcentagem de exchange de Hartree-Fock para os nanotubos (a) $(5,5)$ e (b) $(9,0)$. As linhas são apenas guias para melhor visualização.

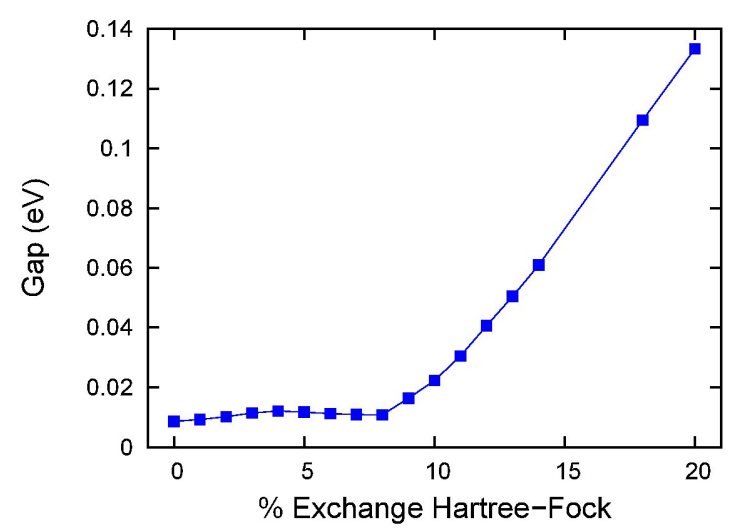

(a)

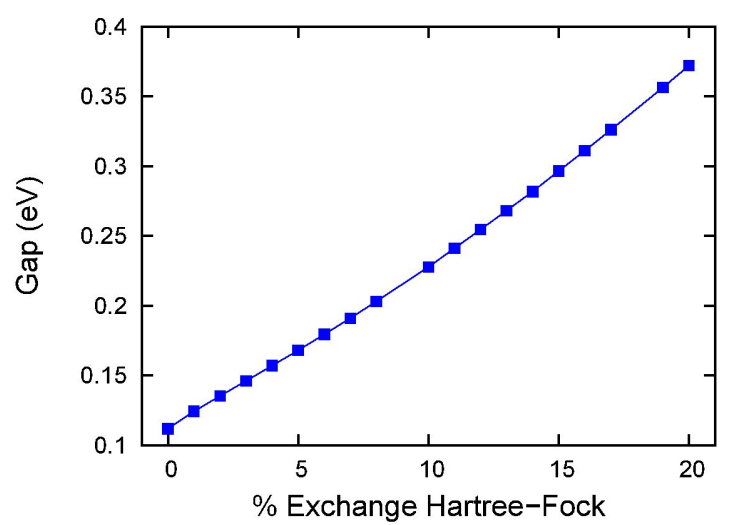

(b)

Figura 5.15.: Gráficos do gap em função da porcentagem de exchange de Hartree-Fock para os nanotubos $(\mathrm{a})(5,5)$ e (b) $(9,0)$. As linhas são apenas guias para melhor visualização.

Tal comportamento para o gap, em conjunto com o fato de que a alternância entre as ligações que se encontram ao longo do eixo dos tubos foi mais acentuada para 
o funcional B3LYP do que para o funcional PBE (tabelas 5.4 e 5.5) evidenciam a distorção de Peierls nestes sistemas.

Para o tubo $(5,5)$, o menor gap direto foi observado no ponto $k=0,36 \pi / a$. Ao triplicar a célula unitária, era esperado que este gap fosse observado para $k=\pi / 3 a$. De fato, o valor observado é uma evidência de que a célula distorcida é na verdade incomensurável com a célula mais simples, ou seja, ela não é um múltiplo inteiro da menor célula unitária.

O tubo $(9,0)$, que deveria apresentar um comportamento condutor, mostrou-se semicondutor, com um gap maior para o B3LYP do que para o PBE. Tal fato pode ser explicado imaginando-se este tubo como uma série de cadeias de cis-policetilenos. Este polímero apresenta um gap intrínseco devido à sua topologia, uma vez que o caminho para sítios equivalentes não é o mesmo nas duas direções da cadeia. 



\section{Resultados Clássicos}

Neste capítulo, as energias de ligação para os nanotubos $(5,5)$ e $(9,0)$ rodeados pelas moléculas de pentaceno são apresentadas, e em seguida, o perfil e a média da energia potencial ao longo da dinâmica para o nanotubo $(5,5)$ na presença de moléculas de pentaceno neutras são apresentados. Os resultados para o mesmo sistema com uma das moléculas de pentaceno carregada e para o nanotubo $(9,0)$ não são apresentados pois apresentaram resultados essencialmente iguais.

\subsection{Mecânica Molecular}

As energias de ligação para o sistema nanotubo $(5,5)$ e pentaceno e nanotubo $(9,0)$ e pentaceno foram calculadas para situações em que haviam 1, 2, 3 ou 4 moléculas de pentaceno ao redor do tubo, com diferentes configurações de posicionamento das moléculas. Utilizando-se as equações 4.1 e 4.2, as energias de ligação foram obtidas e tais valores encontram-se nas tabelas 6.1 e 6.2 .

As figuras ilustrativas do posicionamento das moléculas de pentaceno fora do tubo, juntamente com o código de cada configuração, encontram-se na figuras 6.1, 6.2, 6.3 e 6.4 . 


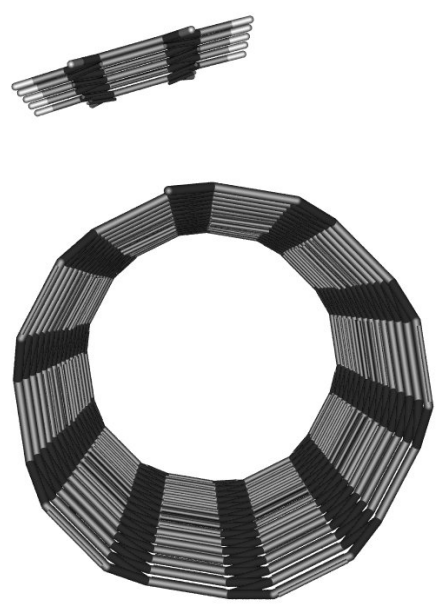

Figura 6.1.: Configuração com 1 molécula de pentaceno próxima à parede externa do tubo

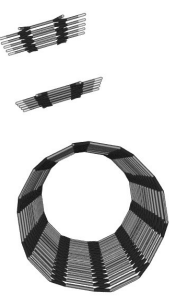

$2 \mathrm{~A}$

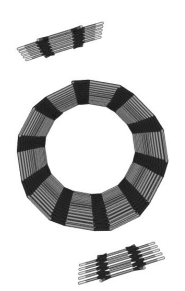

$2 \mathrm{~B}$

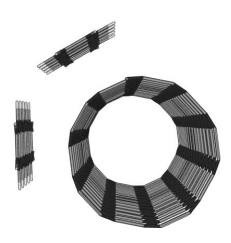

$2 \mathrm{C}$

Figura 6.2.: Configurações com 2 moléculas de pentaceno próximas à parede externa do tubo

\begin{tabular}{lcccccccc} 
Config. & $\mathbf{1}$ & $\mathbf{2 A}$ & $\mathbf{2 B}$ & $\mathbf{2 C}$ & $\mathbf{3 A}$ & $\mathbf{3 B}$ & $\mathbf{3 C}$ & 3D \\
\hline$E_{l i g}$ & $-42,86$ & $-82,47$ & $-98,70$ & $-107,39$ & $-113,79$ & $-131,89$ & $-135,27$ & $-150,88$
\end{tabular}

\begin{tabular}{lccccccc} 
Config. & $\mathbf{4 A}$ & $\mathbf{4 B}$ & $\mathbf{4 C}$ & $\mathbf{4 D}$ & $\mathbf{4 E}$ & $\mathbf{4 F}$ & $\mathbf{4 G}$ \\
\hline$E_{l i g}$ & $-145,20$ & $-141,64$ & $-142,23$ & $-158,19$ & $-160,14$ & $-173,35$ & $-145,95$
\end{tabular}

Tabela 6.1.: Energia de ligação para o sistema $N$ pentacenos + nanotubo $(5,5)$, em $\mathrm{kcal} / \mathrm{mol}$. O código das configurações está explicado nas figuras $6.1,6.2$, 6.3 e 6.4

Pode-se notar que a energia de ligação torna-se mais negativa à medida que as moléculas de pentaceno se posicionam mais próximas da parede externa do tubo e à 


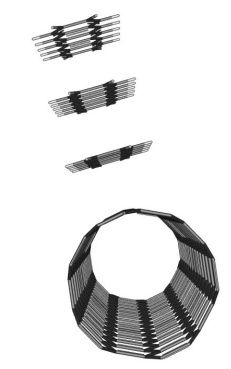

$3 \mathrm{~A}$

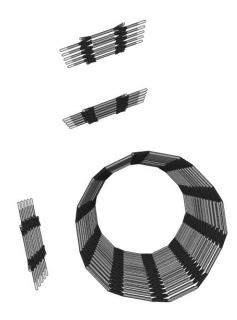

$3 \mathrm{C}$

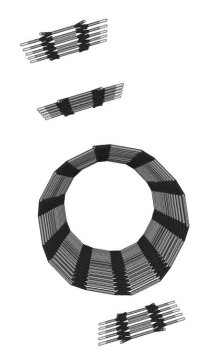

3B

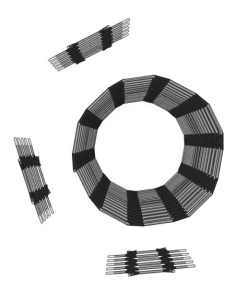

$3 \mathrm{D}$

Figura 6.3.: Configurações com 3 moléculas de pentaceno próximas à parede externa do tubo

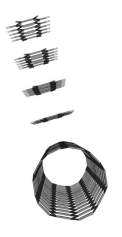

$4 \mathrm{~A}$

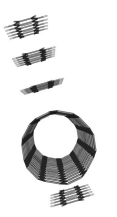

$4 \mathrm{~B}$

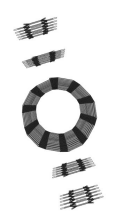

$4 \mathrm{C}$

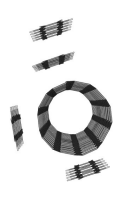

$4 \mathrm{D}$

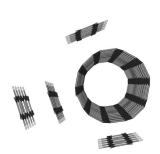

$4 \mathrm{E}$

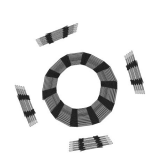

$4 \mathrm{~F}$

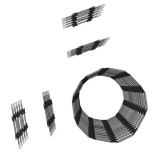

$4 \mathrm{G}$

Figura 6.4.: Configurações com 4 moléculas de pentaceno próximas à parede externa do tubo

medida que estas moléculas aproximam-se umas das outras. 


\begin{tabular}{lcccccccc} 
Config. & $\mathbf{1}$ & $\mathbf{2 A}$ & 2B & 2C & 3A & 3B & 3C & 3D \\
\hline$E_{l i g}$ & $-50,09$ & $-75,28$ & $-93,80$ & $-93,45$ & $-113,54$ & $-132,52$ & $-123,80$ & $-138,11$
\end{tabular}

\begin{tabular}{lccccccc} 
Config. & $\mathbf{4 A}$ & $\mathbf{4 B}$ & $\mathbf{4 C}$ & $\mathbf{4 D}$ & $\mathbf{4 E}$ & $\mathbf{4 F}$ & $\mathbf{4 G}$ \\
\hline$E_{l i g}$ & $-144,37$ & $-148,89$ & $-165,17$ & $-167,97$ & $-169,16$ & $-182,42$ & $-151,73$
\end{tabular}

Tabela 6.2.: Energia de ligação para o sistema $N$ pentacenos + nanotubo $(9,0)$, em $\mathrm{kcal} / \mathrm{mol}$. O código das configurações está explicado nas figuras 6.1, 6.2, 6.3 e 6.4

\subsection{Dinâmica}

Estes resultados no entanto não garantem que estas configurações correspondam a um mínimo global da energia potencial. Para averiguar isto, a dinâmica em $300 \mathrm{~K}$ foi realizada para estas mesmas configurações, e para duas configurações em particular (3A e $4 C$ ), foi observada uma transição de fase, na qual a configuração em que as moléculas de pentaceno estão empilhadas sobre a parade do tudo se mostra instável, dando origem a uma configuração em que as moléculas se posicionam mais próximas à parede do tubo. Isto pode ser observado nos gráficos da figura 6.5.

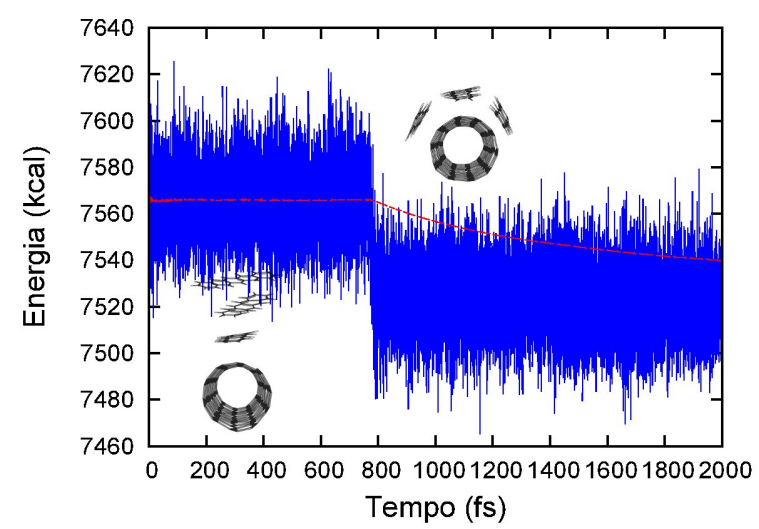

(a)

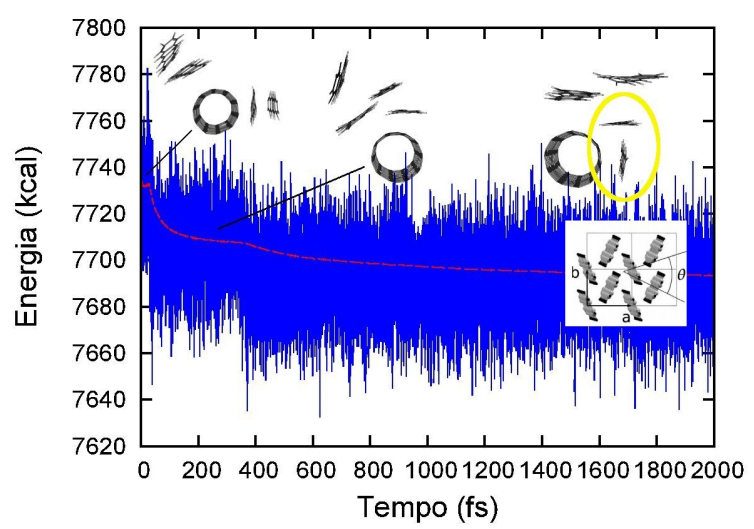

(b)

Figura 6.5.: Gráficos da Energia potencial em função do tempo para o tubo $(5,5)$ com as configurações iniciais $3 A$ (a) e $4 C$ (b). Em (b), é destacada a estrutura cristalina do pentaceno [54]. Em ambas figuras, a linha tracejada representa a média. 
Na figura 6.5 pode-se notar a formação de uma estrutura parecida com a estrutura molecular do cristal de pentaceno - uma estrutura conhecida como "espinha de peixe"1 em que encontramos moléculas posicionadas perpendicularmente a outras, com os eixos maiores paralelos $[54,55]$ - formando-se à direita do tubo, correspondendo à menor energia (figura da direita). A mesma estrutura foi observada em outras dinâmicas, e pode ser considerada um indício de que a formação de cristais de pentaceno próximos à parede do tubo ocorre.

Estes resultados evidenciam uma forte interação entre a parede externa dos nanotubos $(5,5)$ e $(9,0)$ e as moléculas de pentaceno, sendo mais negativa a energia de ligação quanto mais moléculas de pentaceno estiverem próximas à superfície do tubo, sem que haja empilhamento das mesmas. Isso pode ser considerado uma evidência para a formação de aglomerados de pentacenos sobre os tubos.

\footnotetext{
${ }^{1}$ heeringbone em inglês
} 



\section{Conclusões}

Neste trabalho foi estudado o fenômeno da distorção de Peierls para os polímeros cis-poliacetileno e trans-poliacetileno nas simulações com o funcional híbrido B3LYP. O efeito do termo de troca de Hartree-Fock nessa distorção foi avaliado e pôde-se mostrar que quanto maior este termo, maior a distorção da cadeia destes polímeros e consequentemente maior o gap observado. Outro efeito do termo de troca HF foi a diminuição do comprimento da cadeia do polímero e diminuição da energia total por célula unitária à medida que a contribuição do mesmo foi sendo aumentada. Isso foi atribuído ao fato de que este termo diminui a repulsão coulombiana entre elétrons de mesmo spin em orbitais distintos. Os resultados obtidos para o funcional B3LYP foram comparados aos resultados obtidos com o funcional PBE - que não apresentam termo de troca HF - e com valores experimentais para os comprimentos de ligação e gap. Ficou evidenciado que o B3LYP apresenta resultados mais condizentes com o experimento do que o PBE para estes sistemas.

O mesmo fenômeno da distorção de Peierls foi observado para os nanotubos de carbono $(5,5)$ e $(9,0)$, com a observação adicional da diminuição do raio dos tubos com o aumento do termo de troca HF. Isso também pôde ser explicado pela diminuição da repulsão coulombiana introduzida pelo termo de troca de HF. Os resultados obtidos com o B3LYP foram comparados com os resultados para o PBE e foi possível notar que o B3LYP mostrou que para uma célula unitária triplicada, os dois tubos apresentam um comportamento semicondutor, enquanto que para a mesma célula unitária 
triplicada, o PBE mostra um comportamento semicondutor para o tubo $(9,0)$ e um comportamento condutor para o $(5,5)$. Pôde-se mostrar que tal fato está relacionado à distorção das cadeias do nanotubo nesta situação. Comparações com resultados da literatura foram feitas para refutar a idéia de que os tubos armchair são semicondutores apenas quando aglomerados em feixes. [12, 13]

Para a molécula de pentaceno, foi mostrado que o funcional B3LYP apresentou resultados mais próximos aos resultados experimentais de medidas de comprimentos de ligações na molécula do que o funcional PBE.

Em face destes resultados, é possível afirmar que o papel do termo de troca de Hartree-Fock não pode ser desprezado para sistemas deste tipo, uma vez que a diferença mais marcante entre os funcionais utilizados é justamente a presença desse termo. Ao aumentar a participação deste termo, os resultados se aproximaram cada vez mais dos resultados experimentais, sugerindo que o peso deste termo é mais importante que a forma do funcional, pois além desta diferença, os dois funcionais são do tipo GGA.

Finalmente, através do cálculo da energia de ligação entre as moléculas de pentaceno e os tubos utilizando-se o campo de forças clássico CVFF 950 e através da dinâmica molecular clássica com os tubos $(5,5)$ e $(9,0)$ e moléculas de pentaceno ao redor de sua parede externa, utilizando o mesmo campo de forças, foi possível observar indícios de que a formação de cristais de pentaceno é favorecida nesta configuração, pois estruturas do tipo "espinha de peixe"[54, 55] foram observadas durante evolução do sistema. 
Apêndices 



\section{A. Aproximação de Born-Oppenheimer}

A solução exata de um sistema molecular é dada pela equação de Schröedinger independente do tempo

$$
H \Psi(\vec{R}, \vec{r})=E \Psi(\vec{R}, \vec{r})
$$

onde $\vec{R}$ é o conjunto de coordenadas dos núcleos, $\vec{r}$ é o conjunto de coordenadas dos elétrons e o hamiltoniano $H$ é dado por

$$
\begin{aligned}
H= & -\sum_{A=1}^{M} \frac{1}{2 M_{A}} \nabla_{A}^{2}-\frac{1}{2} \sum_{i=1}^{N} \nabla_{i}^{2}-\sum_{i=1}^{N} \sum_{A=1}^{M} \frac{Z_{A}}{\left|\overrightarrow{r_{i}}-\overrightarrow{R_{A}}\right|}+\sum_{i=1}^{N} \sum_{j<i}^{N} \frac{1}{\left|\overrightarrow{r_{i}}-\overrightarrow{r_{j}}\right|}+ \\
& +\sum_{A=1}^{M} \sum_{B<A}^{M} \frac{Z_{A} Z_{B}}{\left|\overrightarrow{R_{A}}-\overrightarrow{R_{B}}\right|} \\
= & T_{N}+T_{e}+V_{N-e}+V_{e-e}+V_{N-N}
\end{aligned}
$$

Colocando o referencial do problema no centro de massa do sistema, esta equação pode ser reescrita como

$$
H=T_{N}+H_{e}+H_{p m}
$$

com

$$
\begin{aligned}
H_{e} & =T_{e}+V_{N-e}+V_{e-e}+V_{N-N} \\
H_{p m} & =-\frac{1}{2 M_{t o t}}\left(\sum_{i=1}^{N} \nabla_{i}\right)^{2}
\end{aligned}
$$

Nesta expressão, $H_{e}$ é o hamiltoniano eletrônico, que além de envolver as coorde- 
nadas e momentos do elétrons do sistema, depende das posições dos núcleos, mas não de seus momentos, e $H_{p m}$ é o operador de polarização de massa. Assumindo que a solução do hamiltoniano eletrônico é dada pelas funções ortonormais $\psi_{i}(\vec{R} ; \vec{r})$, que dependem parametricamente das posições dos núcleos, podemos escrever a equação para os elétrons da seguinte forma

$$
H_{e}(\vec{R}) \psi_{i}(\vec{R} ; \vec{r})=E_{i}(\vec{R} ; \vec{r}) \psi_{i}(\vec{R} ; \vec{r})
$$

em que a dependência paramétrica das coordenadas dos núcleos também está presente no autovalor $E_{i}(\vec{R} ; \vec{r})$ do problema. Com isto, pode-se utilizar uma combinação das soluções para a parte eletrônica como aproximação para a solução do problema

$$
\Psi(\vec{R}, \vec{r})=\sum_{i=1}^{N} \chi_{N i}(\vec{R}) \psi_{i}(\vec{R} ; \vec{r})
$$

Nesta expressão foram utilizadas as funções de onda da parte nuclear $\left(\chi_{N i}\right)$ como coeficientes da expansão das funções $\psi_{i}$. Com isto, a equação de Schröedinger (equação A.1) fica

$$
\sum_{i=1}^{N}\left(T_{N}+H_{e}+H_{p m}\right) \chi_{N i}(\vec{R}) \psi_{i}(\vec{R} ; \vec{r})=E \sum_{i=1}^{N} \chi_{N i}(\vec{R}) \psi_{i}(\vec{R} ; \vec{r})
$$

Usando a notação compacta para o operador energia cinética dos núcleos $T_{N}=\nabla_{N}^{2}$, 
obtemos

$$
\begin{aligned}
& \sum_{i=1}^{N}\left(\nabla_{N}^{2}+H_{e}+H_{p m}\right) \chi_{N i} \psi_{i}=E \sum_{i=1}^{N} \chi_{N i} \psi_{i} \\
& \sum_{i=1}^{N}\left[\nabla_{N}^{2}\left(\chi_{N i} \psi_{i}\right)+H_{e} \chi_{N i} \psi_{i}+H_{p m} \chi_{N i} \psi_{i}\right]=E \sum_{i=1}^{N} \chi_{N i} \psi_{i} \\
& \sum_{i=1}^{N}\left\{\nabla_{N}\left[\left(\psi_{i} \nabla_{N} \chi_{N i}\right)+\left(\chi_{N i} \nabla_{N} \psi_{i}\right)\right]+\chi_{N i} H_{e} \psi_{i}+\chi_{N i} H_{p m} \psi_{i}\right\}=E \sum_{i=1}^{N} \chi_{N i} \psi_{i} \\
& \sum_{i=1}^{N}\left[\psi_{i}\left(\nabla_{N}^{2} \chi_{N i}\right)+2\left(\nabla_{N} \psi_{i}\right)\left(\nabla_{N} \chi_{N i}\right)+\chi_{N i}\left(\nabla_{N}^{2} \psi_{i}\right)+\chi_{N i} E_{i} \psi_{i}+\chi_{N i} H_{p m} \psi_{i}\right]= \\
& \quad=E \sum_{i=1}^{N} \chi_{N i} \psi_{i}
\end{aligned}
$$

Nesta equação, foi usado o fato de que $\nabla_{N}^{2}$ age somente nas coordenadas dos núcleos e $H_{e}$ e $H_{p m}$ agem nas coordenadas eletrônicas. Multiplicando-a por $\psi_{j}^{*}$ e integrando nas coordenadas dos elétrons, obtemos

$$
\begin{aligned}
\nabla_{N}^{2} \chi_{N i} & +E_{j} \chi_{N i}+ \\
& +\sum_{i=1}^{N}\left[2\left\langle\psi_{j}\left|\nabla_{N}\right| \psi_{i}\right\rangle\left(\nabla_{N} \chi_{N i}\right)+\left\langle\psi_{j}\left|\nabla_{N}^{2}\right| \psi_{i}\right\rangle \chi_{N i}+\left\langle\psi_{j}\left|H_{p m}\right| \psi_{i}\right\rangle \chi_{N i}\right]=E \chi_{N j}
\end{aligned}
$$

Os dois primeiros termos entre colchetes são os elementos de acoplamento não adiabático e o último o termo de polarização de massa. Na aproximação adiabática, somente os termos da diagonal destas matrizes são considerados, e com isto resta apenas

$$
\left(\nabla_{N}^{2}+E_{i}+\left\langle\psi_{i}\left|\nabla_{N}^{2}\right| \psi_{i}\right\rangle+\left\langle\psi_{i}\left|H_{p m}\right| \psi_{i}\right\rangle\right) \chi_{N i}=E \chi_{N i}
$$

Considerando-se a massa dos núcleos muito grande $\left(M_{t o t} \rightarrow \infty\right)$, os dois últimos termos dentro dos parênteses podem ser desprezados na aproximação de Born- 
Oppenheimer [41], logo,

$$
\begin{aligned}
& \left(T_{N}+E_{i}\right) \chi_{N i}=E \chi_{N i} \\
& {\left[T_{N}+E_{i}(\vec{R})\right] \chi_{N i}(\vec{R})=E \chi_{N i}(\vec{R})}
\end{aligned}
$$

Com isto, pode-se notar que na equação para os núcleos, o potencial $E_{i}(\vec{R})$ é dado pela solução da equação dos elétrons (equação A.5).

Finalmente, a separação do problema em uma equação para os elétrons (equação A.5) e um equação para os núcleos (equação A.8) é obtida. Na primeira, a dependência com as coordenadas dos núcleos é dada parametricamente e as soluções $\psi_{i}$ são obtidas para um dado conjunto de posições para os núcleos $\left\{\vec{R}_{i}\right\}$. Na última, o potencial ao qual os núcleos estão submetidos é dado pela equação eletrônica. Utilizando-se um método auto consistente, como por exemplo a Teoria do Funcional da Densidade (seção B), é possível obter soluções aproximadas para a parte eletrônica, dada uma configuração para as posições dos núcleos, e com isto, obter a superfície de potencial para os mesmos.

Esta aproximação é geralmente muito boa. Para a molécula de hidrogênio, o erro é da ordem de $10^{-4}$, e a aproximação torna-se melhor à medida que a massa do núcleo aumenta.[41] 


\section{B. Teoria do Funcional da Densidade}

\section{B.1. Teoremas de Hohenberg e Kohn}

A idéia de escrever a energia total de um sistema como funcional da densidade eletrônica do mesmo tem suas origens nos trabalhos independentes de Thomas [56] e Fermi [57] em 1927 e 1928 respectivamente. Somente após muitos anos, em 1964, Hohenberg e Kohn [58] trouxeram de volta estas idéias, propondo os dois teoremas que são considerados os alicerces da Teoria do Funcional da Densidade:

1. O potencial externo $v(\vec{r})$ sentido pelos elétrons é um funcional único da densidade eletrônica $\rho(\vec{r})$ a menos de uma constante.

2. A energia do estado fundamental $E_{0}[\rho]$ é mínima para a densidade $\rho(\vec{r})$ exata.

A prova é feita considerando-se um sistema com N elétrons, cujas posições são dadas pelo conjunto de vetores $\overrightarrow{r_{i}}$.

Seja $\psi$ o estado fundamental do sistema, não degenarado, cujo hamiltoniano é $H=$ $T+V_{e-e}+V_{e x t}$, onde $T$ é a energia cinética, $V_{e-e}$ é a energia de interação elétron-elétron e $V_{\text {ext }}$ é um potencial externo. Seja também $\psi^{\prime}$, o estado fundamental não degenerado de outro sistema, dado pelo hamiltoniano $H^{\prime}=T+V_{e-e}+V_{e x t}^{\prime}$, em que a única diferença para o hamiltoniano anterior é o potencial externo $V_{e x t}^{\prime}$. Por hipótese, considere que 
ambos os potenciais levam à mesma densidade $\rho(\vec{r})$. Pelo teorema variacional [42],

$$
E=\langle\psi|H| \psi\rangle\left\langle\left\langle\psi^{\prime}|H| \psi^{\prime}\right\rangle=\left\langle\psi^{\prime}\left|H^{\prime}\right| \psi^{\prime}\right\rangle+\left\langle\psi^{\prime}\left|V_{e x t}-V_{e x t}^{\prime}\right| \psi^{\prime}\right\rangle\right.
$$

Usando

$$
\rho(\vec{r})=\left\langle\psi\left|\sum_{i=1}^{N} \delta\left(\vec{r}-\overrightarrow{r_{i}}\right)\right| \psi\right\rangle \quad V_{e x t}=\sum_{i=1}^{N} v\left(\overrightarrow{r_{i}}\right)
$$

obtemos

$$
\begin{aligned}
\left\langle\psi\left|V_{\text {ext }}\right| \psi\right\rangle & =\sum_{i=1}^{N} \int d^{3} r_{1} \ldots \int d^{3} r_{N} \psi^{*}\left(\overrightarrow{r_{1}}, \ldots, \overrightarrow{r_{N}}\right) v\left(\overrightarrow{r_{i}}\right) \psi\left(\overrightarrow{r_{1}}, \ldots, \overrightarrow{r_{N}}\right) \\
& =\sum_{i=1}^{N} \int d^{3} r \int d^{3} r_{1} \ldots \int d^{3} r_{i} v(\vec{r}) \delta\left(\vec{r}-\overrightarrow{r_{i}}\right) \int d^{3} r_{i+1} \ldots \int d^{3} r_{N} \psi^{*} \psi \\
& =\int \rho(\vec{r}) v(\vec{r}) d^{3} r
\end{aligned}
$$

Utilizando $V_{e x t}^{\prime}$ em B.2, a equação B.1 fica

$$
E<E^{\prime}+\int\left[v(\vec{r})-v^{\prime}(\vec{r})\right] d^{3} r
$$

Fazendo o mesmo para $\left\langle\psi^{\prime}\left|H^{\prime}\right| \psi^{\prime}\right\rangle$, chegamos em

$$
E^{\prime}<E+\int\left[v^{\prime}(\vec{r})-v(\vec{r})\right] d^{3} r
$$

Somando-se as equações B.3 e B.4, chega-se ao absurdo

$$
E+E^{\prime}<E+E^{\prime}
$$

Com isto, conclui-se que para que os diferentes $\psi$ 's possam forncer a mesma densidade $\rho$, eles devem ser iguais, ou seja, a densidade do estado fundamental deve conter as mesmas informações que a função de onda do mesmo. Com isto o primeiro teorema está provado. 
O segundo teorema é provado considerando-se duas densidades, $\rho$ e $\rho_{0}$, que são a densidade de um estado $\psi$ qualquer e a densidade de um estado $\psi_{0}$, estado fundamental do problema, respectivamente. Com isto,

$$
\begin{aligned}
E[\rho] & =\left\langle\psi\left|T+V_{e-e}\right| \psi\right\rangle+\left\langle\psi\left|V_{e x t}\right| \psi\right\rangle \\
& =F[\rho]+\left\langle\psi\left|V_{e x t}\right| \psi\right\rangle
\end{aligned}
$$

onde $F[\rho]$ é um funcional universal válido para qualquer sistema coulombiano e $\left\langle\psi\left|V_{\text {ext }}\right| \psi\right\rangle$ depende do sistema.

A equação B.6 também é válida para $\rho_{0}$, fornecendo

$$
\begin{aligned}
E\left[\rho_{0}\right] & =\left\langle\psi_{0}\left|T+V_{e-e}\right| \psi_{0}\right\rangle+\left\langle\psi_{0}\left|V_{e x t}\right| \psi_{0}\right\rangle \\
& =F\left[\rho_{0}\right]+\left\langle\psi_{0}\left|V_{e x t}\right| \psi_{0}\right\rangle
\end{aligned}
$$

Como $\rho_{0}$ determina $\psi_{0}$ e $\rho$ determina $\psi$, e como $\rho$ e $\rho_{0}$ são determinados por algum potencial externo, ou seja, são $v$-representáveis, pode-se aplicar o teorema variacional, obtendo-se

$$
\begin{gathered}
E\left[\psi_{0}\right]<E[\psi] \\
\left\langle\psi_{0}\left|T+V_{e-e}\right| \psi_{0}\right\rangle+\left\langle\psi_{0}\left|V_{e x t}\right| \psi_{0}\right\rangle<\left\langle\psi\left|T+V_{e-e}\right| \psi\right\rangle+\left\langle\psi\left|V_{e x t}\right| \psi\right\rangle \\
F\left[\rho_{0}\right]+\left\langle\rho_{0}\left|V_{e x t}\right| \rho_{0}\right\rangle<F[\rho]+\left\langle\psi\left|V_{e x t}\right| \psi\right\rangle \\
E\left[\rho_{0}\right]<E[\rho]
\end{gathered}
$$

Com isto completa-se a prova do segundo teorema.

\section{B.2. Equações de Kohn-Sham}

Substituindo a equação B.2 em B.6, e escrevendo a parte de $F[\rho]$ correspondente às interações coulombianas explicitamente, pelo fato das mesmas serem de longo alcance, 
temos que B.6 fica

$$
E[\rho]=\int v(\vec{r}) \rho(\vec{r}) d \vec{r}+\frac{1}{2} \iint \frac{\rho(\vec{r}) \rho\left(\overrightarrow{r^{\prime}}\right)}{\left|\vec{r}-\overrightarrow{r^{\prime}}\right|} d \vec{r} d \overrightarrow{r^{\prime}}+G[\rho]
$$

Com $G[\rho]=T_{0}[\rho]+E_{x c}[\rho]$ um funcional universal da densidade eletrônica, constituído da parte da energia cinética e da parte de Troca-Correlação. Minimizando $E[\rho]$ sujeito ao vínculo de que o número de elétrons do sistema permanece inalterado, ou seja,

$$
\int \rho(\vec{r}) d \vec{r}=N
$$

tem-se

$$
\begin{gathered}
\delta\left\{E[\rho]-\mu\left[\int \rho(\vec{r}) d \vec{r}-N\right]\right\}=0 \\
\int \delta \rho(\vec{r})\left[v(\vec{r})+\frac{1}{2} \int \frac{\rho\left(\overrightarrow{r^{\prime}}\right)}{\left|\vec{r}-\overrightarrow{r^{\prime}}\right|} d \overrightarrow{r^{\prime}}+\frac{\delta G[\rho]}{\delta \rho}-\mu\right] d \vec{r}=0
\end{gathered}
$$

Mas no entanto, não se sabia como a energia cinética dependia da densidade, assim como não se conhecia a forma do funcional de troca e correlação, logo, não era possível tratar o termo $\delta G / \delta \rho$ devidamente.

Em seu trabalho de 1965, Kohn e Sham [59] partiram da fórmula exata da energia cinética do estado fundamental,

$$
T_{s}=-\frac{1}{2} \sum_{i=1}^{N}\left\langle\psi_{i}\left|\nabla^{2}\right| \psi_{i}\right\rangle
$$

e propuseram construir a densidade eletrônica a partir de funções $\psi_{i}$,

$$
\rho(\vec{r})=\sum_{i=1}^{N}\left|\psi_{i}(\vec{r})\right|^{2}
$$

e também consideraram um sistema de referência não interagente, cuja função de onda no estado fundamental seria

$$
\Psi_{s}=\frac{1}{\sqrt{N !}} \operatorname{det}\left[\psi_{1} \psi_{2} \ldots \psi_{N}\right]
$$


Com isto, a energia cinética deste sistema seria

$$
\begin{aligned}
T_{s}[\rho] & =\left\langle\Psi_{s}\left|\sum_{i=1}^{N}-\frac{1}{2} \nabla_{i}^{2}\right| \Psi_{s}\right\rangle \\
& =-\frac{1}{2} \sum_{i=1}^{N}\left\langle\psi_{i}\left|\nabla_{i}^{2}\right| \psi_{i}\right\rangle
\end{aligned}
$$

Assim seria possível, rearranjar o problema de modo que $T_{s}[\rho]$ fosse exatamente a parte da energia cinética do funcional $G[\rho]$ de interesse

$$
G[\rho]=T_{s}[\rho]+\frac{1}{2} \iint \frac{\rho(\vec{r}) \rho\left(\overrightarrow{r^{\prime}}\right)}{\left|\vec{r}-\overrightarrow{r^{\prime}}\right|} d \vec{r} d \overrightarrow{r^{\prime}}+E_{x c}[\rho]
$$

$\operatorname{com} E_{x c}[\rho]=T[\rho]-T_{s}[\rho]+V_{e-e}[\rho]-K[\rho]$. Com isto, obtiveram então

$$
E[\rho]=-\frac{1}{2} \sum_{i=1}^{N} \int \psi_{i}^{*}(\vec{r}) \nabla^{2} \psi_{i}(\vec{r}) d \vec{r}+\int \rho(\vec{r}) v(\vec{r}) d \vec{r}+\frac{1}{2} \iint \frac{\rho(\vec{r}) \rho\left(\overrightarrow{r^{\prime}}\right)}{\left|\vec{r}-\overrightarrow{r^{\prime}}\right|} d \vec{r} d \overrightarrow{r^{\prime}}+E_{x c}[\rho]
$$

Kohn e Sham então introduziram a função

$$
\Omega\left[\left\{\psi_{i}\right\}\right]=E[\rho]-\sum_{i j} \epsilon_{i j}\left\langle\psi_{i} \mid \psi_{j}\right\rangle
$$

e a minimizaram, variando-se os $\psi_{i}$ com a restrição $\left\langle\psi_{i} \mid \psi_{j}\right\rangle=\delta_{i j}$, obtendo

$$
\int \delta \psi_{i}^{*}(\vec{r})\left[-\frac{1}{2} \nabla^{2}+v(\vec{r})+\frac{1}{2} \int \frac{\rho\left(\overrightarrow{r^{\prime}}\right)}{\left|\vec{r}-\overrightarrow{r^{\prime}}\right|} d \overrightarrow{r^{\prime}}+v_{x c}(\vec{r})-\sum_{j} \epsilon_{i j}\right] \psi_{j}(\vec{r}) d \vec{r}+\text { C.C. }=0
$$

cujas soluções são as funções $\psi_{i}$ conhecidas como orbitais de Kohn e Sham, dadas por

$$
\left[-\frac{1}{2} \nabla^{2}+v_{K S}(\vec{r})\right] \psi_{i}(\vec{r})=\sum_{j} \epsilon_{i j} \psi_{j}(\vec{r})
$$

Finalmente, fazendo uma mudança de base de modo que a matriz dos coeficientes $\epsilon_{i j}$ seja diagonal, obtemos as equações de Kohn-Sham.

$$
\left[-\frac{1}{2} \nabla^{2}+v_{K S}(\vec{r})\right] \psi_{i}(\vec{r})=\epsilon_{i} \psi_{i}(\vec{r})
$$


Tais equações são resolvidas para seus autovetores $\psi_{i}$ e autovalores $\epsilon_{i}$ através de um método autoconsistente. Este método consiste em um "chute"inicial da função $\rho(\vec{r})$, para que se possa construir

$$
v_{K S}(\vec{r})=v(\vec{r})+\frac{1}{2} \int d^{3} \overrightarrow{r^{\prime}} \frac{\rho\left(\overrightarrow{r^{\prime}}\right)}{\left|\vec{r}-\overrightarrow{r^{\prime}}\right|}+v_{x c}[\rho(\vec{r})]
$$

com isto resolve-se a equação B.11 e com o conjunto de $\psi_{i}$ 's obtidos, constrói-se uma nova densidade eletrônica através de B.9 e utiliza-se esta nova densidade para se resolver as equações B.11 novamente. O processo é repetido até que critérios de convergência sejam alcançados para a densidade $\rho(\vec{r})$, de acordo com o fluxograma da figura B.1.

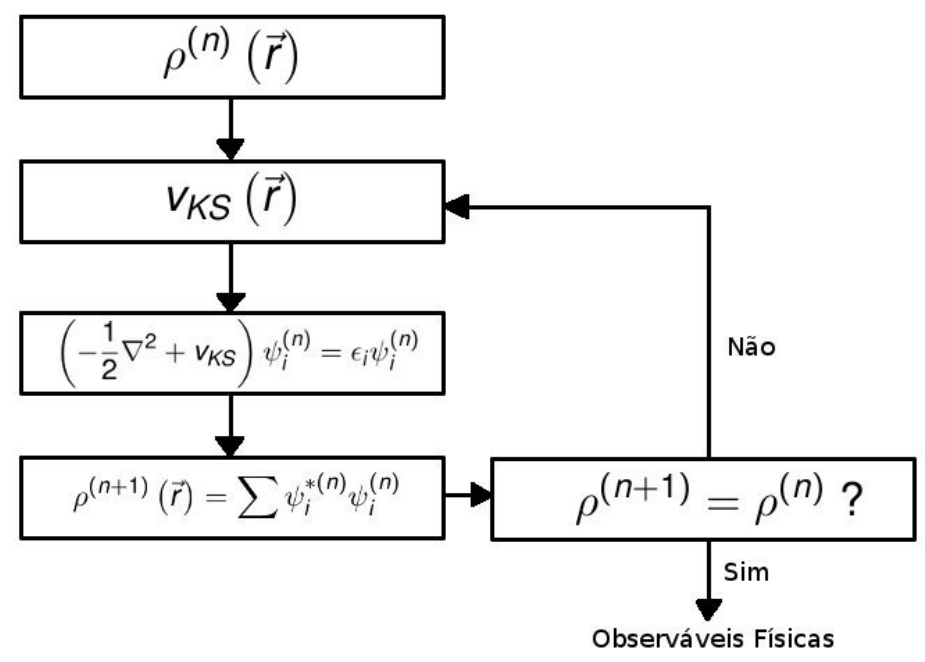

Figura B.1.: Fluxograma explicativo do processo autoconsistente do DFT

No entanto, a forma exata do termo de troca e correlação (último termo da equação B.12) não é conhecida, sendo alvo de constantes esforços a busca de formas funcionais para este termo. 


\section{B.3. Funcionais da DFT}

A primeira proposta para o termo de troca e correlação ficou conhecida como LDA ${ }^{1}$. Nesta aproximação, assume-se que a energia de troca e correlação do sistema de interesse é igual à energia de troca e correlação de um gás de elétrons homogêneo de igual densidade, ponto a ponto, e que a densidade varia suavemente nas proximidades do ponto onde é calculada. Com isto, pode-se escrever $E_{x c}[\rho]$ como

$$
E_{x c}^{L D A}[\rho]=\int \rho(\vec{r}) \epsilon_{x c}^{h}(\rho(\vec{r})) d^{3} r
$$

$\operatorname{com} \epsilon_{x c}^{h}$ a energia de troca e correlação por elétron de um gás de elétrons homogêneo de densidade $\rho$.

Tal aproximação mostra-se boa para sistemas que apresentam características de um gás de elétrons livres, como por exemplo metais, mas no caso de compostos orgânicos, em que a densidade eletrônica apresenta variações consideráveis, a aproximação LDA não apresenta bons resultados. Um exemplo disto é o cálculo da estrutura de bandas do poliacetileno (figura 2.2), que na aproximação LDA apresenta um gap muito menor do que o esperado, evidenciando um comportamento metálico, não condizente com os resultados experimentais. [48]

Um refinamento da aproximação LDA foi a utilização de um funcional que levasse em consideração também o gradiente da densidade eletrônica, o que ficou conhecido como aproximação GGA (Generalized Gradient Approximation), cuja fórmula funcional é dada por

$$
E_{x c}^{G G A}[\rho]=\int f(\rho(\vec{r}), \nabla \rho(\vec{r})) d^{3} r
$$

Diversos funcionais foram propostos dentro da aproximação GGA, entre os mais

\footnotetext{
${ }^{1}$ Aproximação de Densidade Local - Local Density Approach
} 
famosos, o de Perdew-Burke-Erzenhof (PBE) [38], Lee-Yang-Parr-Becke (BLYP) [60] entre outros.

Apesar de representar um avanço da DFT, por tornar os resultados da teoria mais próximos dos resultados experimentais, a aproximação GGA não dava conta de todas as discrepâncias, e foi Becke em 1993 [39] que mostrou que o uso de funcionais híbridos, em que parte da energia de troca e correlação é dada pelo termo de troca de HartreeFock (equação B.15) resultava em valores melhorados para energia de dissociação de diversos sistemas.

$$
K_{i j}=\left\langle\phi_{i}\left(\overrightarrow{r_{1}}\right) \phi_{j}\left(\overrightarrow{r_{2}}\right)\left|\frac{1}{\left|\overrightarrow{r_{1}}-\overrightarrow{r_{2}}\right|}\right| \phi_{i}\left(\overrightarrow{r_{2}}\right) \phi_{j}\left(\overrightarrow{r_{1}}\right)\right\rangle
$$

Neste trabalho, foi proposto o funcional B3LYP, em que 3 parâmetros definem o peso dos termos de troca de Slater, troca de Hartree-Fock, troca de Becke e correlação de Lee-Yang-Parr no funcional (primeiro, segundo, terceiro e últimos termos em B.16).

$$
E_{x c}^{B 3 L Y P}=a E_{x}^{\text {Slater }}+(1-a) E_{x}^{\text {Hartree-Fock }}+b \Delta E_{x}^{\text {Becke }}+E_{x}^{L D A}+c \Delta E_{c}^{\text {Lee-Yang-Parr }}
$$

com $a, b$ e $c$ parâmetros a serem ajustados. 


\section{Condições periódicas de contorno}

Devido à simetria translacional dos sistemas periódicos, tanto em 1 dimensão quanto em 2 ou 3 dimensões, a função de onda dos elétrons na aproximação de elétrons independentes deste sistema deve satisfazer o teorema de Bloch [28] [9],

$$
\psi\left(\vec{r}+\vec{a}_{i}\right)=e^{i \vec{k} \cdot \vec{a}_{i}} \psi(\vec{r})
$$

onde $\vec{a}_{i}$ é um vetor da rede direta e $\vec{k}$ é um vetor no espaço recíproco.

Uma possibilidade para construir esta função é a utilização de uma combinação linear de orbitais atômicos do tipo

$$
\phi_{j}(\vec{k}, \vec{r})=\frac{1}{\sqrt{N}} \sum_{c=1}^{N} e^{i \vec{k} \cdot \vec{R}_{c}} \chi_{j}\left(\vec{r}-\vec{R}_{a}-\vec{R}_{c}\right)
$$

que descreve a função de onda do orbital do estado $j$, centrado no átomo localizado em $\vec{R}_{a}$ dentro da célula unitária $c$. A soma se estende pelas $N$ células unitárias consideradas no problema. Com isso, a função de onda do $j$-ésimo orbital cristalino do sistema fica dada por

$$
\psi_{j}(\vec{k}, \vec{r})=\sum_{i=1}^{n} c_{i j}(\vec{k}) \phi_{i}(\vec{k}, \vec{r})
$$

com os coeficientes $c_{i j}(\vec{k})$ a serem determinados. 
Os elementos de matriz de um operador $A$ com essas funções são dados por

$$
\begin{aligned}
A_{m n} & =\frac{1}{N} \sum_{c, c^{\prime}=1}^{N} e^{i \vec{k} \cdot\left(\vec{R}_{c}-\vec{R}_{c^{\prime}}\right)} \int d^{3} \vec{r} \chi_{m}^{*}\left(\vec{r}-\vec{R}_{a}-\vec{R}_{c}\right) A \chi_{n}\left(\vec{r}-\vec{R}_{a^{\prime}}-\vec{R}_{c^{\prime}}\right) \\
& =\frac{1}{N} N \sum_{c=1}^{N} e^{i \vec{k} \cdot \vec{R}_{c}} \int d^{3} \vec{r} \chi_{m}^{*}\left(\vec{r}-\vec{R}_{a}+\vec{R}_{a^{\prime}}-\vec{R}_{c}\right) A \chi_{n}(\vec{r}) \\
& =\sum_{c=1}^{N} e^{i \vec{k} \cdot \vec{R}_{c}} A_{n m}\left(\vec{R}_{a}, \vec{R}_{a^{\prime}}, \vec{R}_{c}\right)
\end{aligned}
$$

onde utilizou-se o fato de que o operador deve ser invariante por translação e que uma das somas nas células unitárias pode ser substituida por $N$ devido à simetria de translação da rede.

De fato, este tipo de função pode ser utilizado em diversos tipos de cálculos em que se utiliza funções de partículas independentes, como no modelo Tight Binding, Hartree-Fock ou mesmo para cálculos de DFT com funcionais híbridos.

\section{C.1. Tight-Binding}

Utilizando-se o método variacional [31][42], uma aproximação ${ }^{1}$ para a energia do j-ésimo orbital cristalino de um sistema periódico e para os coeficientes do autovetor correpodente a este orbital são obtidos a partir de

$$
E_{j}(\vec{k})=\frac{\left\langle\Psi_{j}|H| \Psi_{j}\right\rangle}{\left\langle\Psi_{j} \mid \Psi_{j}\right\rangle}
$$

Utilizando-se as funções de onda da equação C.3, a equação C.5 fica

$$
E_{j}(\vec{k})=\frac{\sum_{p q}^{n} c_{j p}^{*} c_{j q}\left\langle\Phi_{p}|H| \Phi_{q}\right\rangle}{\sum_{p q}^{n} c_{j p}^{*} c_{j q}\left\langle\Phi_{p} \mid \Phi_{q}\right\rangle}=\frac{\sum_{p q}^{n} c_{j p}^{*} c_{j q} H_{p q}(\vec{k})}{\sum_{p q}^{n} c_{j p}^{*} c_{j q} S_{p q}(\vec{k})}
$$

onde $H_{p q}(\vec{k})=\left\langle\Phi_{p}(\vec{k})|H| \Phi_{q}(\vec{k})\right\rangle$ e $S_{p q}(\vec{k})=\left\langle\Phi_{p}(\vec{k}) \mid \Phi_{q}(\vec{k})\right\rangle$ são dados pela equação C.4.

\footnotetext{
${ }^{1} \mathrm{O}$ método variacional fornece um limite superior para a energia dos orbitais [31][42].
} 
Para $\vec{k}$ fixo, a energia variacional apresenta um mínimo, ou seja,

$$
\begin{aligned}
\frac{\partial E_{i}(\vec{k})}{\partial c_{i j}^{*}} & =\frac{\sum_{p}^{n} c_{i p} H_{j p}}{\sum_{p q}^{n} c_{i p}^{*} c_{i q} S_{p q}}-\frac{\sum_{p q}^{n} c_{i p}^{*} c_{i q} H_{p q} \sum_{r}^{n} c_{i r} S_{j r}}{\left(\sum_{p q}^{n} c_{i p}^{*} c_{i q} S_{p q}\right)^{2}} \\
& =\sum_{p=1}^{n} c_{i p} H_{j p}-E_{i}(\vec{k}) \sum_{r=1}^{n} c_{i r} S_{j r} \\
& =0
\end{aligned}
$$

onde foi utilizado o fato de que o denominador de C.6 é não nulo e substituiu-se $E_{i}(\vec{k})$ de C.6 no segundo termo. Finalmente, isso pode ser reescrito como

$$
\sum_{p=1}^{n}\left[H_{j p}(\vec{k})-E_{i}(\vec{k}) S_{j p}(\vec{k})\right] c_{p i}=0
$$

Este sistema linear pode ser expresso como o produto entre a matriz $H-E_{i} S$ e o vetor $c_{i}$, e apresenta solução não trivial apenas se $\operatorname{det}(H-E S) \neq 0$. Obtendo-se os autovalores e autovetores deste sistema, as energias $E_{i}(\vec{k})$ dos orbitais cristalinos e seus coeficientes $c_{i j}(\vec{k})$ são obtidos, para cada vetor de onda $\vec{k}$ particular.

\section{C.2. DFT híbrido com condições periódicas}

As funções de onda que descrevem um sistema periódico, dadas pela equação C.3 podem ser utilizadas também para cálculos de maior complexidade que não lançam mão de parametrizações como a que foi feita na seção anterior. Um exemplo é a utilização das mesmas em cálculos baseados na DFT com funcionais híbridos, como o B3LYP.

De acordo com a equação B.16, o funcional B3LYP apresenta uma contribuição do termo de troca de Hartree-Fock, dado por B.15. Utilizando as funções periódicas C.3 
para reescrever este termo, temos que o mesmo é dado pela soma dos termos [61]

$$
k_{i j}^{c}=-\sum_{c^{\prime} c^{\prime \prime}=1}^{N} \sum_{p q} P_{p q}^{c^{\prime} c^{\prime \prime}} \int d^{3} \vec{r}_{1} d^{3} \vec{r}_{2} \chi_{i}^{*}\left(\vec{r}_{1}\right) \chi_{p}\left(\vec{r}_{1}-\vec{R}_{c^{\prime}}\right) \frac{1}{\left|\vec{r}_{1}-\vec{r}_{2}\right|} \chi_{j}^{*}\left(\vec{r}_{2}-\vec{R}_{c}\right) \chi_{q}\left(\vec{r}_{2}-\vec{R}_{c^{\prime \prime}}\right)
$$

com $P_{p q}^{c^{\prime} c^{\prime \prime}}$ os elementos da matriz de densidade que conecta dois orbitais, $p$ e $q$ localizados nas células unitárias $c^{\prime}$ e $c^{\prime \prime}$ respectivamente, dados por

$$
P_{p q}^{c^{\prime} c^{\prime \prime}}=\sum_{\vec{k}}^{B . Z \cdot} \sum_{n}^{\text {ocup. }} e^{-i \vec{k} \cdot\left(\vec{R}_{c^{\prime}}-\vec{R}_{c^{\prime \prime}}\right)} c_{p n}^{*}(\vec{k}) c_{q n}(\vec{k})
$$

A condição de neutralidade da carga no sistema é dada por [61]

$$
2 \sum_{c}^{N} \sum_{i j} P_{i j}^{0 c} S_{i j}^{0 c}=\sum_{z} Z_{a}
$$

Com isto, as equações de Kohn-Sham (equação B.11) podem ser resolvidas através do processo autoconsistente ilustrado na figura B.1. A diferença neste caso é que neste caso, a energia dos orbitais de Kohn-Sham e os coeficientes dos autovetores da equação serão funções de $\vec{k}$. Neste caso, são escolhidos alguns valores para $\vec{k}$ e estas grandezas são resolvidas para cada valor, e com isto é possível obter grandezas como a estruturas de bandas dos sistemas periódicos.

Uma questão que deve ser analisada com muito cuidado no processo de obtenção dos elementos de matriz é em que ponto que a soma nas células unitárias deve ser truncada. Tal truncamento deve ser feito quando os termos das séries que determinam os elementos de matriz são considerados pequenos, ou seja, quando o valor numérico das integrais envolvidas é menor que um certo limite. Para sistemas como o poliacetileno, Suhai [61] mostrou que as integrais de overlap $S$ e de energia cinética caem exponencialmente, e segundo o critério adotado por ele, o raio de corte para estes elementos deveria ser entre 9 e 10 Åpara uma base STO-3G. Os elementos da matriz densidade para o mesmo sistema deveriam ser somados até termos que correspondessem a uma 
distância de 38 a 40 Å. A matriz correspondente ao termo de troca de Hartree-Fock, por apresentar um decaimento proporcional a $1 / r^{2}$, deveria ter seu raio de corte em torno de $40 \AA$.

Finalmente, a tarefa mais complicada é escolher um raio de corte que satisfaça a condição de neutralidade de carga (equação C.11), uma vez que o truncamento da série no ponto incorreto faria com que a densidade de carga eletrônica integrada não compensasse a carga dos núcleos da célula unitária. Isso se torna particularmente complicado à medida que funções difusas (orbitais do tipo $d$ no carbono, por exemplo) são adicionadas ao problema. [44] 



\section{Métodos clássicos}

\section{D.1. Campos de Forças}

Os núcleos são pesados o bastante para que na maioria das vezes os efeitos quânticos possam ser desprezados e os átomos sejam tratados como partículas clássicas. De fato, se os efeitos quânticos fossem importantes para os núcleos, a própria idéia de geometria molecular deveria ser abandonada, uma vez que o princípio da incerteza de Heisenberg não permite o conhecimento das posições nucleares com uma precisão menor do que as dimensões moleculares. No entanto, alguns sistemas como por exemplo a molécula de amônia podem apresentar efeitos quânticos em sua estrutura, como por exemplo o tunelamento do átomo de nitrogênio através do plano determinado pelos 3 átomos de hidrogênio desta última.

Neste caso, a dinâmica dos átomos, que são considerados esferas duras nesta aproximação, é dada então pela segunda lei de Newton

$$
-\vec{\nabla} V(\vec{R})=M \frac{d^{2} \vec{R}}{d t^{2}}
$$

Nos métodos de campos de forças, o potencial $V$ é dado por uma função paramétrica das coordenadas dos átomos. Os parâmetros são obtidos tanto de resultados experimentais quanto de simulações.[62]

Os campos de forças contém os componentes necessários para o cálculo da energia e da força agindo nos núcleos: 
- Uma lista de tipos de átomos.

- Uma lista de cargas dos átomos (se não estiver inclusa no item anterior).

- Regras de classificação dos átomos.

- Formas funcionais dos componentes da expressão da energia potencial.

- Regras para gerar parâmetros omitidos e regras para atribuir formas funcionais e parâmetros

Com estes componentes, é possível escrever uma expressão para a energia do campo de forças como uma soma de termos.

$$
E_{c f}=E_{e s t}+E_{a n g}+E_{\text {torc }}+E_{f p}+E_{v d W}+E_{C}+E_{c r u z}
$$

Os termos $E_{\text {est }}, E_{\text {ang }}, E_{\text {torc }}$ e $E_{f p}$ são os termos da energia provenientes do estiramento de ligações, de dobramento entre duas ligações, de torção ou rotação ao redor do eixo das ligações e interações fora do plano. O termo $E_{\text {cruz }}$ corresponde ao acoplamento entre estes três termos, e os termos $E_{v d W}$ e $E_{C}$ correspondem aos termos não-ligados provenientes da interação de van der Waals e Coulomb, respectivamente.

O termo de estiramento é expresso usualmente como um potencial harmônico,

$$
E_{e s t}=\sum_{i<j} \alpha_{i j}\left(R_{i j}-R_{i j}^{0}\right)^{2}
$$

onde $\alpha_{j i}$ é a constante de força da interação entre os átomos ligados $i$ e $j$, que é nula no caso de um par não ligado. $R_{i j}^{0}$ é a distância de equilíbrio entre núcleos. No entanto, um potencial também bastante usado é o potencial de Morse,

$$
E_{e s t}=\sum_{i<j} \alpha_{i j}\left[1-e^{-\beta_{i j}\left(R_{i j}-R_{i j}^{0}\right)}\right]^{2}
$$

onde os parâmetros $\alpha_{i j}, \beta_{i j}$ e $R_{i j}^{0}$ são dados para os pares ligados. 
O termo angular envolve trios de átomos ligados formando um ângulo entre as duas ligações, e é comumente expresso por um termo harmônico.

$$
E_{\text {ang }}=\sum_{i<j<k} \alpha_{i j k}\left(\theta_{i j k}-\theta_{i j k}^{0}\right)^{2}
$$

O termo de torção envolve 4 átomos, ligados de modo que a rotação das duas ligações periféricas ao redor do eixo da ligação central possa ser medida através do ângulo $\omega_{i j k l}$, de acordo com a figura D.1

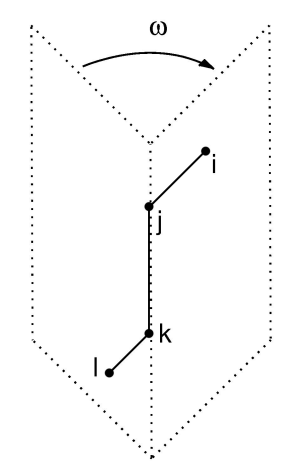

Figura D.1.: Esquema ilustrativo do ângulo de torção $\omega$.

A expressão do termo de torção da energia geralmente é dada por uma função periódica,

$$
E_{\text {torc }}=\sum_{n, i j k l} \gamma_{i j k l}\left[1+\cos \left(n \omega_{i j k l}\right)\right]
$$

onde os átomos $i, j, k$ e $l$ na soma devem estar ligado de acordo com a figura D.1.

O termo $E_{f p}$ envolve deformações fora do plano, como por exemplo entre uma ligação de um átomo de carbono com hibridização $s p^{2}$ e o plano determinado pelos orbitais hibridizados, como ilustrado na figura D.2.

Este termo da energia é geralmente expresso como uma função harmônica, com a mesma forma da expressão do termo angular (equação D.5) envolvendo no entanto 4 átomos ao invés de 3 . Outros termos de deformação fora do plano também são 


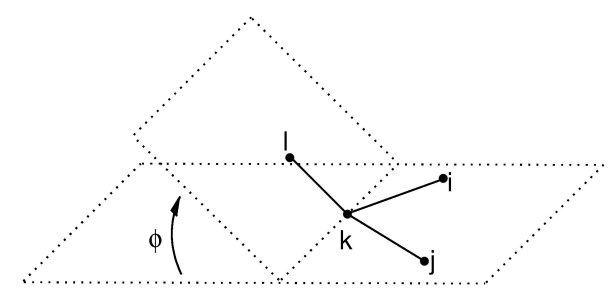

Figura D.2.: Esquema ilustrativo de deformação fora do plano.

considerados, como as inversões umbrella e impróprias, cujas expressões não serão ilustradas aqui, podendo ser obtidas da literatura [62].

Os termos cruzados são simplesmente a mistura das formas funcionais dos termos vistos anteriormente, como por exemplo entre o estiramento e a torção,

$$
E_{\text {cruz }}^{\text {est-torc }}=\sum \alpha_{i j}\left(R_{i j}-R_{i j}^{0}\right) \gamma_{i j k l}\left[1+\cos \left(n \omega_{i j k l}\right)\right]
$$

ou também com termos de mesma origem, mas oriundos de conjuntos de átomos diferentes, como por exemplo o termo cruzado que acopla dois estiramentos de ligações distintas,

$$
E_{c r u z}^{e s t-e s t}=\sum \alpha_{i j} \alpha_{j k}\left(R_{i j}-R_{i j}^{0}\right)\left(R_{j k}-R_{j k}^{0}\right)
$$

Finalmente, os termos não ligados de van der Waals e Coulomb são dados pelas equações D.9 e D.10 a seguir.

$$
\begin{gathered}
E_{v d W}=\sum_{i<j}\left[\frac{A_{i j}}{R_{i j}^{12}}-\frac{B_{i j}}{R_{i j}^{6}}\right] \\
E_{C}=\sum_{i<j} \frac{Z_{i} Z_{j}}{R_{i j}}
\end{gathered}
$$

\section{D.2. Minimização de energia}

Dada a forma funcional da energia potencial, o passo seguinte é a obtenção da geometria de menor energia do sistema. Isto é obtido calculando-se o gradiente da energia 
potencial em relação a todas as coordenadas das posições dos átomos, e deslocando os átomos de acordo com a força exercida nos mesmos. Um dos algoritmos mais conhecidos, a busca por linha[62], calcula o valor da energia ao longo de uma linha dada pelo gradiente,

$$
\overrightarrow{R^{\prime}}=\vec{R}+\left.\alpha \vec{\nabla} E\right|_{\vec{R}}
$$

O valor do mínimo de energia é obtido para um ponto $\overrightarrow{R^{\prime}}$ sobre esta linha e novamente o gradiente é calculado, obtendo-se uma nova linha e a mesma é percorrida da mesma forma. O processo é repetido até que seja encontrada uma linha em que a mudança na energia seja menor que a tolerância pré estabelecida.

\section{D.3. Dinâmica}

A dinâmica molecular clássica consiste em obter as trajetórias no espaço de fase de um sistema composto por átomos. A interação entre os átomos é dada pelo campo de forças utilizado, que fornece as componentes da energia potencial $V$ do sistema, e com isto as equações de movimento são obtidas através de

$$
m_{i} \frac{d^{2} x_{i}}{d t^{2}}=-\frac{\partial V}{\partial x_{i}}=F_{i}
$$

onde o índice $i$ corresponde a uma das $3 N$ coordenadas do problema de $N$ átomos.

Estas equações podem ser integradas utilizando-se o artifício de expandir as coordenadas em série de Taylor da seguinte forma

$$
x_{i}\left(t^{\prime} \pm \Delta t\right)=x_{i}\left(t^{\prime}\right) \pm\left.\frac{d x_{i}}{d t}\right|_{t^{\prime}} \Delta t+\frac{F_{i}\left(t^{\prime}\right)}{m_{i}} \frac{\Delta t^{2}}{2 !} \pm\left.\frac{d^{3} x_{i}}{d t^{3}}\right|_{t^{\prime}} \frac{\Delta t^{3}}{3 !}+\mathcal{O}\left(\Delta t^{4}\right)
$$

Somando-se a expansão de sinal positivo com a de sinal negativo, obtém-se

$$
x_{i}(t+\Delta t)=2 x_{i}(t)-x_{i}(t-\Delta t)+\frac{F_{i}(t)}{m} \Delta t^{2}+\mathcal{O}\left(\Delta t^{4}\right)
$$

Este algoritmo, que é do tipo Verlet [62], fornece as posições em função do tempo 
com um erro da ordem de $\Delta t^{4}$ sem que haja necessidade do conhecimento das velocidades.

As velocidades podem ser obtidas através de

$$
v_{i}(t-\Delta t / 2)=\frac{x_{i}(t)-x_{i}(t-\Delta t)}{\Delta t} \quad v_{i}(t+\Delta t / 2)=\frac{x_{i}(t+\Delta t)-x_{i}(t)}{\Delta t}
$$

Note que as velocidades são determinadas com uma defasagem de $\Delta t / 2$ em relação às posições. Isso não é um problema pois as velocidades no instante $t$ podem ser obtidas através da média

$$
v_{i}(t)=\frac{v_{i}(t+\Delta t / 2)+v_{i}(t-\Delta t / 2)}{2}
$$

Este algoritmo apresenta uma boa conservação de energia para a ordem de algunas dezenas de passos de integração e um baixo desvio para algo em torno de alguns milhares de passos. [63]

Para manter a energia constante, de modo que a dinâmica ocorra no ensemble NVT, um método de controle da temperatura conhecido como termostato deve empregado. Neste trabalho, o termostato de Nosé-Hoover foi utilizado. [64][65] 


\section{Referências Bibliográficas}

[1] S Iijima. Helical microtubules of graphitic carbon. Nature, 354:56-58, 1991.

[2] Sander J. Tans, Alwin R. M. Verschueren, and Cees Dekker. Room-temperature transistor based on a single carbon nanotube. Nature, 393(6680):49 - 52, 1998. doi: 10.1038/29954. URL http://dx.doi.org/10.1038/29954.

[3] Sander J. Tans, Michel H. Devoret, Hongjie Dai, Andreas Thess, Richard E. Smalley, L. J. Geerligs, and Cees Dekker. Individual single-wall carbon nanotubes as quantum wires. Nature, 386(6624):474 - 477, 1997. doi: 10.1038/386474a0. URL http://dx.doi.org/10.1038/386474a0.

[4] Henk W. Ch. Postma, Tijs Teepen, Zhen Yao, Milena Grifoni, and Cees Dekker. Carbon nanotube single-electron transistors at room temperature. Science, 293(5527):76-79, 2001. doi: 10.1126/science.1061797. URL http: //www.sciencemag.org/content/293/5527/76. abstract.

[5] R. Martel, T. Schmidt, H. R. Shea, T. Hertel, and Ph. Avouris. Single- and multi-wall carbon nanotube field-effect transistors. 73(17): 2447-2449, 1998. ISSN 00036951. doi: DOI:10.1063/1.122477. URL http://dx.doi.org/doi/10.1063/1.122477.

[6] Zhen Yao, Henk W. Ch. Postma, Leon Balents, and Cees Dekker. Carbon 
nanotube intramolecular junctions. Nature, 402(6759):273 - 276, 1999. doi: 10.1038/46241. URL http://dx.doi.org/10.1038/46241.

[7] Adrian Bachtold, Peter Hadley, Takeshi Nakanishi, and Cees Dekker. Logic circuits with carbon nanotube transistors. Science, 294(5545):1317-1320, 2001. doi: 10.1126/science.1065824. URL http://www . sciencemag. org/content/294/5545/1317 . abstract.

[8] M. S. Fuhrer, J. Nygård, L. Shih, M. Forero, Young-Gui Yoon, M. S. C. Mazzoni, Hyoung Joon Choi, Jisoon Ihm, Steven G. Louie, A. Zettl, and Paul L. McEuen. Crossed nanotube junctions. Science, 288(5465):494-497, 2000. doi: 10.1126/science.288.5465.494. URL http://www. sciencemag.org/content/288/5465/494 . abstract.

[9] R Saito, G Dresselhaus, and M S Dresselhaus. Physical Properties of Carbon Nanotubes. Imperial College Press, 1998.

[10] Kenji Okahara, Kazuyoshi Tanaka, Hiroo Aoki, Tohru Sato, and Tokio Yamabe. Band structures of carbon nanotubes with bondalternation patterns. Chemical Physics Letters, 219(5-6):462 - 468, 1994. ISSN 0009-2614. doi: DOI: 10.1016/0009-2614(94)00091-3. URL http://www.sciencedirect.com/science/article/pii/0009261494000913.

[11] Paul Delaney, Hyoung Joon Choi, Jisoon Ihm, Steven G. Louie, and Marvin L. Cohen. Broken symmetry and pseudogaps in ropes of carbon nanotubes. Nature, 391(6666):466 - 468, 1998. doi: 10.1038/35099. URL http://dx.doi.org/10.1038/35099.

[12] S. Reich, C. Thomsen, and P. Ordejón. Electronic band structure of isolated 
and bundled carbon nanotubes. Phys. Rev. B, 65(15):155411, Mar 2002. doi: 10.1103/PhysRevB.65.155411.

[13] Min Ouyang, Jin-Lin Huang, Chin Li Cheung, and Charles M. Lieber. Energy gaps in "metallic"single-walled carbon nanotubes. Science, 292(5517):702-705, 2001. doi: 10.1126/science.1058853. URL http: //www . sciencemag. org/content/292/5517/702. abstract.

[14] P. M. Albrecht and J. W. Lyding. Ultrahigh-vacuum scanning tunneling microscopy and spectroscopy of single-walled carbon nanotubes on hydrogenpassivated si(100) surfaces. 83(24):5029-5031, 2003. ISSN 00036951. doi: DOI:10.1063/1.1633014. URL http://dx.doi.org/doi/10.1063/1.1633014.

[15] J. W. Mintmire, B. I. Dunlap, and C. T. White. Are fullerene tubules metallic? Phys. Rev. Lett., 68(5):631-634, Feb 1992. doi: 10.1103/PhysRevLett.68.631.

[16] Marc Thilo Figge, Maxim Mostovoy, and Jasper Knoester. Peierls transition with acoustic phonons and solitwistons in carbon nanotubes. Phys. Rev. Lett., 86(20): 4572-4575, May 2001. doi: 10.1103/PhysRevLett.86.4572.

[17] Takashi Yumura, Kaori Hirahara, Shunji Bandow, Kazunari Yoshizawa, and Sumio Iijima. A theoretical study on the geometrical features of finite-length carbon nanotubes capped with fullerene hemisphere. Chemical Physics Letters, 386(1-3): 38 - 43, 2004. ISSN 0009-2614. doi: DOI: 10.1016/j.cplett.2003.12.123. URL http://www.sciencedirect.com/science/article/pii/S0009261404000478.

[18] D. Connétable, G.-M. Rignanese, J.-C. Charlier, and X. Blase. Room temperature peierls distortion in small diameter nanotubes. Phys. Rev. Lett., 94(1):015503, Jan 2005. doi: 10.1103/PhysRevLett.94.015503. 
[19] Rudolf E. Peierls. Quantum Theory of Solids - Oxford Classics Series. Oxford University Press, 2001.

[20] C. J. Drury, C. M. J. Mutsaers, C. M. Hart, and M. Matters an d D. M. de Leeuw. Low-cost all-polymer integrated circuits. Applied Physics Letters, 73(1):108-110, 1998. doi: 10.1063/1.121783. URL http://link.aip.org/link/?APL/73/108/1.

[21] Kazuhito Tsukagoshi, Masahiro Sekiguchi, Yoshinobu Aoyagi, Takayoshi Kanbara, Taishi Takenobu, and Yoshihiro Iwasa. Suppression of current hysteresis in carbon nanotube thin-film transistors. Japanese Journal of Applied Physics, 46(23):L571-L573, 2007. doi: 10.1143/JJAP.46.L571. URL http://jjap.ipap.jp/link?JJAP/46/L571/.

[22] Woong Kim, Ali Javey, Ophir Vermesh, Qian Wang, Yiming Li, and Hongjie Dai. Hysteresis caused by water molecules in carbon nanotube field-effect transistors. Nano Letters, 3(2):193-198, 2003. doi: 10.1021/n10259232. URL http://pubs.acs.org/doi/abs/10.1021/n10259232.

[23] Shuhong Liu, Stefan C. B. Mannsfeld, Melbs C. LeMieux, Hang W. Lee, and Zhenan Bao. Organic semiconductor-carbon nanotube bundle bilayer field effect transistors with enhanced mobilities and high on/off ratios. $A p$ plied Physics Letters, 92(5):053306, 2008. doi: 10.1063/1.2841033. URL http: //link.aip.org/link/?APL/92/053306/1.

[24] Linus Pauling. The nature of the chemical bond. application of results obtained from the quantum mechanics and from a theory of paramagnetic susceptibility to the structure of molecules. Journal of the American Chemi- 
cal Society, 53(4):1367-1400, 1931. doi: doi: 10.1021/ja01355a027. URL http://dx.doi.org/10.1021/ja01355a027.

[25] D. E. Resasco, W.E. Alvarez, F. Pompeo, L. Balzano, J.E. Herrera, B. Kitiyanan, and A. Borgna. A scalable process for production of single-walled carbon nanotubes (swnts) by catalytic disproportionation of co on a solid catalyst. Journal of Nanoparticle Research, 4:131-136, 2002. doi: 10.1023/A:1020174126542. URL http: //www. springerlink. com/content/T642W241LL741366.

[26] H. W. Kroto, J. R. Heath, S. C. O'Brien, R. F. Curl, and R. E. Smalley. C60: Buckminsterfullerene. Nature, 318:162-163, 1985. doi: 10.1038/318162a0. URL http://dx.doi.org/10.1038/318162a0.

[27] A. K. Geim and K. S. Novoselov. The rise of graphene. Nat Mater, 6:183-191, 2007. doi: 10.1038/nmat1849. URL http://dx.doi.org/10.1038/nmat1849.

[28] Charles Kittel. Introduction to Solid State Physics. John Wiley \& Sons, inc, $8^{\text {th }}$ edition, 2005.

[29] W. P. Su, J. R. Schrieffer, and A. J. Heeger. Solitons excitations in polyacetilene. Phys. Rev. B, 22(4):2099-2111, August 1980.

[30] J Oshika. J. Phys. Soc. Jpn., 12:1238-1246, 1957.

[31] J. J. Sakurai. Modern Quantum Mechanics - Revised Edition. Addison-Wesley Publishing Company, 1994.

[32] S. J. Park, H. W. Yeom, S. H. Min, D. H. Park, and I. W. Lyo. Direct evidence of the charge ordered phase transition of indium nanowires on si(111). Phys. Rev. Lett., 93(10):106402, Sep 2004. doi: 10.1103/PhysRevLett.93.106402. 
[33] M. J. Frisch and et al. Gaussian 03 revision e.01, 2004. URL http://www.gaussian.com.

[34] M. J. Frisch and et al. Gaussian 03 revision a.02, 2009. URL http://www.gaussian.com.

[35] Cerius2, version 4.10, 1995. URL http://www.accelrys.com.

[36] Zs. Szekeres, F. Bogár, and J. Ladik. B3lyp, blyp and pbe dft band structures of the nucleotide base stacks. International Journal of Quantum Chemistry, 102(4):422-426, 2005. ISSN 1097-461X. doi: 10.1002/qua.20394. URL http://dx.doi.org/10.1002/qua. 20394.

[37] M. De La Pierre, R. Orlando, L. Maschio, K. Doll, P. Ugliengo, and R. Dovesi. Performance of six functionals (lda, pbe, pbesol, b3lyp, pbe0, and wc1lyp) in the simulation of vibrational and dielectric properties of crystalline compounds. the case of forsterite mg2sio4. Journal of Computational Chemistry, 32(9):1775-1784, 2011. ISSN 1096-987X. doi: 10.1002/jcc.21750. URL http://dx.doi.org/10.1002/jcc.21750.

[38] John P. Perdew, Kieron Burke, and Matthias Ernzerhof. Generalized gradient approximation made simple. Phys. Rev. Lett., 77(18):3865-3868, Oct 1996. doi: 10.1103/PhysRevLett.77.3865.

[39] A. D. Becke. Density-functional thermochemistry. iii. the role of exact exchange. J. Chem. Phys., 98(7):5648-5652, 1993.

[40] Melvin E. Zandler and Francis DŚouza. The remarkable ability of b3lyp $/ 3-21 g(*)$ calculations to describe geometry, spectral and electrochemical properties of molecular and supramolecular porphyrin- 
fullerene conjugates. Comptes Rendus Chimie, 9(7-8):960 - 981, 2006. ISSN 1631-0748. doi: DOI: 10.1016/j.crci.2005.12.008. URL http: //www.sciencedirect.com/science/article/pii/S1631074805003929. Chime des fullerenes.

[41] Frank Jensen. Introduction to Computational Chemistry. John Wiley \& Sons, inc, 1999 .

[42] Adalberto Fazzio, José David M. Vianna, and Sylvio Canuto. Teoria Quântica de Moléculas e Sólidos. Editora Livraria da Física, 2004.

[43] Gaussian 09 help table of contents. URL http://www.gaussian.com/g_tech/g_ur/g09help.htm.

[44] Cristian V. Diaconu. Guide for pbc calculations with gaussian. URL http://scuseria.rice.edu/gau/gdv_pbc/pbc_guide.html\#hse_thresh.

[45] Konstantin N. Kudin and Gustavo E. Scuseria. A fast multipole algorithm for the efficient treatment of the coulomb problem in electronic structure calculations of periodic systems with gaussian orbitals. Chem. Phys. Lett., 289:611-616, 1998.

[46] Konstantin N. Kudin and Gustavo E. Scuseria. Linear-scaling density-functional theory with gaussian orbitals and periodic boundary conditions: Efficient evaluation of energy and forces via the fast multipole method. Phys. Rev. B, 61(24): $16440-16453,1999$.

[47] P. Dauber-Osguthorpe, V. A. Roberts, D. J. Osguthorpe, J. Wolff, M. Genest, and A. T. Hagler. Structure and energetics of ligand binding to proteins: Escherichia coli dihydrofolate reductase-trimethoprim, a drug-receptor system. Proteins: Structure, Function and Genetics, 4(1):31-47, 1988. 
[48] So Hirata, Hajime Torii, and Mitsuo Tasumi. Density-functional crystal orbital study on the structures and energetics of polyacetylene isomers. Phys. Rev. B, 57 (19):11994-12001, May 1998. doi: 10.1103/PhysRevB.57.11994.

[49] Eugene S. Kadantsev, M. J. Stott, and Angel Rubio. Electronic structure and excitations in oligoacenes from ab initio calculations. The Journal of Chemical Physics, 124(13):134901, 2006. doi: 10.1063/1.2186999. URL http://link.aip.org/link/? JCP/124/134901/1.

[50] H. Kataura, Y. Kumazawa, Y. Maniwa, I. Umezu, S. Suzuki, Y. Ohtsuka, and Y. Achiba. Optical properties of single-wall carbon nanotubes. Synthetic Metals, 103(1-3):2555 - 2558, 1999. ISSN 0379-6779. doi: DOI: 10.1016/S0379-6779(98)00278-1. URL http://www.sciencedirect.com/science/article/pii/S0379677998002781. International Conference on Science and Technology of Synthetic Metals.

[51] Ch. Kramberger, R. Pfeiffer, H. Kuzmany, V. Zólyomi, and J. Kürti. Assignment of chiral vectors in carbon nanotubes. Phys. Rev. B, 68(23):235404, Dec 2003. doi: $10.1103 /$ PhysRevB.68.235404.

[52] M.S. Dresselhaus, G. Dresselhaus, R. Saito, and A. Jorio. Raman spectroscopy of carbon nanotubes. Physics Reports, 409(2):47 - 99, 2005. ISSN 0370-1573. doi: DOI: 10.1016/j.physrep.2004.10.006. URL http://www.sciencedirect.com/science/article/pii/S0370157304004570.

[53] J. Kürti, V. Zólyomi, M. Kertesz, G. Sun, R. H. Baughman, and H. Kuzmany. Individualities and average behavior in the physical properties of small diameter single-walled carbon nanotubes. Carbon, 42(5-6):971 - 978, 2004. ISSN 0008-6223. doi: DOI: 10.1016/j.carbon.2003.12.029. URL 
http://www.sciencedirect.com/science/article/pii/S0008622303005827. European Materials Research Society 2003, Symposium B: Advanced Multifunctional Nanocarbon Materials and Nanosystems.

[54] Dmitrii Nabok, Peter Puschnig, Claudia Ambrosch-Draxl, Oliver Werzer, Roland Resel, and Detlef-M. Smilgies. Crystal and electronic structures of pentacene thin films from grazing-incidence x-ray diffraction and first-principles calculations. Phys. Rev. B, 76(23):235322, Dec 2007. doi: 10.1103/PhysRevB.76.235322.

[55] R. B. Campbell, J. Monteath Robertson, and J. Trotter. The crystal and molecular structure of pentacene. Acta. Cryst., 14:705, 1961.

[56] L. H. Thomas. The calculation of atomic fields. Mathematical Proceedings of the Cambridge Philosophical Society, 23(05):542-548, 1927. doi: $10.1017 / \mathrm{S} 0305004100011683$.

[57] E. Fermi. Z. Physik, 48:73, 1928.

[58] P. Hohenberg and W. Kohn. Inhomogeneous electron gas. Phys. Rev., 136(3B): B864-B871, Nov 1964. doi: 10.1103/PhysRev.136.B864.

[59] W. Kohn and L. J. Sham. Self-consistent equations including exchange and correlation effects. Phys. Rev., 140(4A):A1133-A1138, Nov 1965. doi: 10.1103/PhysRev.140.A1133.

[60] Chengteh Lee, Weitao Yang, and Robert G. Parr. Development of the collesalvetti correlation-energy formula into a functional of the electron density. Phys. Rev. B, 37(2):785-789, Jan 1988. doi: 10.1103/PhysRevB.37.785.

[61] S. Suhai. A priori electronic structure calculations on highly conducting polymers. 
i. hartree-fock studies on cis- and trans-polyacetylenes (polyenes). J. Chem. Phys., 73(8):3843-3853, 1980.

[62] Cerius2 forcefield based simulations. URL http://www.chem.cmu.edu/courses/09-560/docs/msi/ffbsim/FF_SimulTOC.doc.html.

[63] D Frenkel and B Smit. Understanding molecular simulation: from algorithms to applications. Academic Press, San Diego, 1996.

[64] M P Allen and D J Tildesley. Computer simulation of liquids. University Press, Oxford, 1989.

[65] W G Hoover. Canonical dynamics: Equilibrium phase-space distributions. Phys. Rev. A, 31:1695-1697, 1985. 